\title{
Manifestations of the Electron-Phonon Coupling in the Spectroscopy of High-Temperature Superconductors
}

\author{
A. S. Mishchenko ${ }^{1,2}$ \\ ${ }^{1}$ Cross-Correlated Materials Research Group (CMRG), ASI, RIKEN, Wako 351-0198, Japan \\ ${ }^{2}$ Russian Research Centre, Kurchatov Institute, Moscow 123182, Russia \\ Correspondence should be addressed to A. S. Mishchenko, mishchenko@riken.jp
}

Received 28 June 2009; Accepted 28 July 2009

Academic Editor: Sasha Alexandrov

Copyright ( 2010 A. S. Mishchenko. This is an open access article distributed under the Creative Commons Attribution License, which permits unrestricted use, distribution, and reproduction in any medium, provided the original work is properly cited.

\begin{abstract}
A review of experimental and theoretical results on the spectroscopy of high-temperature superconductors is presented. The models where hole doped into antiferromagnet interacts both with magnetic subsystem and with phonons are considered. Theoretical results of these models for phonon spectra, angle resolved photoemission spectra, and optical conductivity are presented. Comparison with experimental data gives evidence for the strong electron-phonon coupling in the undoped and weakly doped high-temperature superconductors. The strength of electron-phonon coupling decreases with doping though at the optimal doping the compounds are still in the intermediate coupling regime.
\end{abstract}

\section{Introduction}

The role of the electron-phonon interaction (EPI) in the high-temperature superconductors is debated for many years. Firm opinions range from the complete negation of its role [1] up to the statement that the polaron binding energy is larger than the characteristic magnetic energy by an order of magnitude and, hence, the magnetic subsystem is not important $[2,3]$. Of course there is an opinion that both magnetic and lattice subsystems are important [4].

Up to the very recent times it seemed that the spectral properties of cuprates can be explained considering only magnetic subsystem. The dispersion of the peak in the Angle Resolved Photoemission (ARPES) spectra was well reproduced by $\mathrm{tt}^{\prime} \mathrm{t}^{\prime \prime}$-J model [5] which is the model with a hole moving on the antiferromagnetic background [6-8]. Also, t-J model predicted a peak in the Optical Conductivity (OC) [9-22], situated roughly at the same energy as the Mid Infrared (MIR) band observed in experiments [23-28]. However, improvement of the experimental technique and advances in theoretical methods make them capable of seeing fine details of spectra which occurred to be quite different in experiment and theory. In experiment, the resolution of ARPES technique reached few $\mathrm{meV}$ [5] and the ellipsometry technique $[29,30]$ gave possibility to measure OC without Kramers-Kronig relation which introduce uncertainty into the experimental data. In theory, recently developed Diagrammatic Monte Carlo (DMC) method [31-56] became capable of avoiding serious approximations in a significant set of models describing strongly correlated systems.

One of the main and most evident contradiction between theory and experiment was the linewidth of the ARPES peak in undoped cuprates. Although the dispersion of the peak is well reproduced by $\mathrm{tt}^{\prime} \mathrm{t}^{\prime \prime}-\mathrm{J}$ model, its width is very broad in experiment [57] and very narrow in theory [37]. Naively, contribution of EPI cannot explain the large width since the coupling to phonons, in addition to broadening, must also change the dispersion of the particle which, in turn, is already well described by the pure $\mathrm{tt}^{\prime} \mathrm{t}^{\prime \prime}-\mathrm{J}$ model. However, as was shown in $[42,58]$, in the strong coupling regime of EPI the situation is exactly the same as in experiment. The polaron quasiparticle has very small weight and cannot be seen in experiment while shake-off Franck-Condon peak completely reproduces the dispersion of the pure magnetic model without EPI. Naturally, in such case the chemical potential must be pinned not to the observed broad shake-off peak but to the real invisible quasiparticle. Such decoupling of the chemical potential from the broad peak was observed 
in experiment [59] a few months after prediction had been made in [42].

Further theoretical and experimental studies brought more evidences of the importance of EPI in cuprates. One of the evidences is the two-peak structure of the MIR part of OC in the underdoped compounds which is easily reproduced by taking the EPI into account [51]. Another confirmation is the anomalous temperature dependence of the width of the ARPES peak which can be explained only by the interplay of magnetic and lattice system $[50,60]$. Various estimates for the EPI strength [50, 61-63] give the value $\lambda \approx 1$ for undoped compounds. The strength of EPI decreases with increase of the concentration of holes reaching the intermediate coupling regime at optimal doping $[51,64]$.

In Section 2 we introduce the models describing the physics of the interplay between magnetic and lattice degrees of freedom in cuprates. Then, we discuss different aspects of manifestation of the EPI in the phonon spectra, ARPES, and $\mathrm{OC}$ in Section 3. The influence of strong electronic correlations on the EPI is discussed in Section 4 and the final conclusions are presented in Section 5 .

\section{Models}

The prototypical model for high-temperature superconductors is the three band model [65]. This model contains one $3 d x^{2}-y^{2}$ orbital of $\mathrm{Cu}$ and two oxygen $p$ orbitals in the $\mathrm{CuO}_{2}$ plain. The Hamiltonian

$$
\begin{aligned}
\hat{H}_{3 \mathrm{~B}}= & \varepsilon_{\mathrm{O}} \sum_{\mathbf{i} \delta \sigma} a_{\mathbf{i} \delta \sigma}^{\dagger} a_{\mathbf{i} \delta \sigma}+\varepsilon_{d} \sum_{\mathbf{i} \sigma} c_{\mathbf{i} \sigma}^{\dagger} c_{\mathbf{i} \sigma} \\
& +U \sum_{\mathbf{i}} n_{\mathbf{i} \uparrow} n_{\mathbf{i} \downarrow}+t_{p d} \sum_{\mathbf{i} \delta \sigma} P_{\delta}\left(c_{\mathbf{i} \sigma}^{\dagger} a_{\mathbf{i} \delta \sigma}+\text { h.c. }\right)
\end{aligned}
$$

includes term with Coulomb repulsion $U$ on $\mathrm{Cu}$ ion and term describing hopping between $\mathrm{Cu}$ and $\mathrm{O}$ with amplitude $t_{p d}$. The vector $\boldsymbol{\delta}$ defines oxygen position in the unit cell and runs over $(a / 2,0)$ and $(0, a / 2)$ in the second term and over $( \pm a / 2,0)$ and $(0, \pm a / 2)$ in the last term. $P_{\delta}=-P_{-\delta}$ is even operator. Operator $a_{\mathrm{i} \delta \sigma}^{\dagger}\left(c_{\mathrm{i} \sigma}^{\dagger}\right)$ is the creation operator of electron with spin $\sigma$ on the oxygen (cooper) ion. Energy $\varepsilon_{d}$ $\left(\varepsilon_{O}\right)$ corresponds to $\mathrm{Cu}(\mathrm{O})$ orbital.

The $\mathrm{t}-\mathrm{J}$ model is derived from the three-band model. The site $i$ in the $\mathrm{t}-\mathrm{J}$ model corresponds to the position of cooper ion. In the undoped system all $\mathrm{Cu}$ ions have configuration $d^{9}$ and each site is occupied by one hole. Doped holes go mostly to the $\mathrm{O}$ sites and form with hole the Zhang-Rice singlet [66]. This singlet is an empty state in the t-J model described by the following Hamiltonian:

$$
\hat{H}_{\mathrm{t}-\mathrm{J}}=-t \sum_{\langle\mathbf{i j}\rangle \sigma}\left(\tilde{c}_{\mathbf{i} \sigma}^{\dagger} \tilde{c}_{\mathbf{j} \sigma}+\text { h.c. }\right)+J \sum_{\langle\mathbf{i j}\rangle}\left(\mathbf{S}_{\mathbf{i}} \mathbf{S}_{\mathbf{j}}+\frac{n_{\mathbf{i}} n_{\mathbf{j}}}{4}\right) .
$$

Here $\widetilde{c}_{\mathrm{j} \sigma}$ is projected (to avoid double occupancy) fermionic annihilation operator, $n_{\mathbf{i}}<2$ is the number operator, $\mathbf{S}_{\mathbf{i}}$ is spin $1 / 2$ operator, $J$ is the exchange integral, and $\langle\mathbf{i j}\rangle$ is restricted to near neighbors in two-dimensional lattice.

Expressing spin operators in terms of spin waves and making Fourier and Bogoliubov transformations one can derive the t-J Hamiltonian in the spin wave approximation [22, 67-71]. Adding hoppings to the second $t^{\prime}$ and third $t^{\prime \prime}$ near neighbors $t^{\prime}$ and $t^{\prime \prime}[6-8,72-76]$ one arrives to the Hamiltonian of the $t t^{\prime} t^{\prime \prime}-\mathrm{J}$ model (3) in the spin wave approximation. A hole ( $h_{\mathbf{k}}$ is its annihilation operator) with dispersion $\varepsilon(\mathbf{k})=4 t^{\prime} \operatorname{cox}\left(k_{x}\right) \cos \left(k_{y}\right)+2 t^{\prime \prime}\left[\cos \left(2 k_{x}\right)+\right.$ $\left.\cos \left(2 k_{y}\right)\right]$ moves in the field of magnons $\left(\alpha_{\mathbf{k}}\right.$ is annihilation operator of the magnon):

$$
\hat{H}_{\mathrm{t}-\mathrm{J}}^{0}=\sum_{\mathbf{k}} \varepsilon(\mathbf{k}) h_{\mathbf{k}}^{\dagger} h_{\mathbf{k}}+\sum_{\mathbf{k}} \omega_{\mathbf{k}} \alpha_{\mathbf{k}}^{\dagger} \alpha_{\mathbf{k}}
$$

The dispersion of magnons is $\omega_{\mathbf{k}}=2 J \sqrt{1-\gamma_{\mathbf{k}}^{2}}$, where $\gamma_{\mathbf{k}}=$ $\left(\cos k_{x}+\cos k_{y}\right) / 2$. The hole is scattered by magnons

$$
\hat{H}_{\mathrm{t}-\mathrm{J}}^{\mathrm{h}-\mathrm{m}}=N^{-1 / 2} \sum_{\mathbf{k}, \mathbf{q}} M_{\mathbf{k}, \mathbf{q}}\left[h_{\mathbf{k}}^{\dagger} h_{\mathbf{k}-\mathbf{q}} \alpha_{\mathbf{k}}+\text { h.c. }\right]
$$

with the scattering vertex $M_{\mathbf{k}, \mathbf{q}}$. Amplitudes $t, t^{\prime}$, and $t^{\prime \prime}$ describe hoppings to the nearest, next nearest, and next next nearest neighbors. The simplest t-J model corresponds to the case when $t^{\prime}$ and $t^{\prime \prime}$ are set to zero. For hole (electron) doping the signs of the hopping amplitudes are $t>0, t^{\prime}<0$, and $t^{\prime \prime}>0\left(t<0, t^{\prime}>0, t^{\prime \prime}<0\right)[76-79]$. Note that $\mathrm{t}-\mathrm{J}$ model can be also derived from the Hubbard model $[80,81]$

$$
H_{\mathrm{H}}=-t \sum_{\langle\mathbf{i j}\rangle \sigma}\left(\tilde{c}_{\mathbf{i} \sigma}^{\dagger} \widetilde{c}_{\mathbf{j} \sigma}+\text { h.c. }\right)+U \sum_{\mathbf{i}} n_{\mathbf{i} \uparrow} n_{\mathbf{i} \downarrow}
$$

with large $U \rightarrow \infty$.

In the generalized $\mathrm{tt}^{\prime} \mathrm{t}^{\prime \prime}-\mathrm{J}$-Holstein $\left(\mathrm{tt}^{\prime} \mathrm{t}^{\prime \prime}-\mathrm{J}-\mathrm{H}\right)$ model the short range EPI with dispersionless phonons with frequency $\Omega_{0}$,

$$
\hat{H}^{\mathrm{ph}}=\Omega_{0} \sum_{\mathbf{k}} b_{\mathbf{k}}^{\dagger} b_{\mathbf{k}},
$$

is described by Holstein Hamiltonian:

$$
\hat{H}^{\mathrm{e}-\mathrm{ph}}=N^{-1 / 2} \sum_{\mathbf{k}, \mathbf{q}} \frac{\sigma}{\sqrt{2 M \Omega_{0}}}\left[h_{\mathbf{k}}^{\dagger} h_{\mathbf{k}-\mathbf{q}} b_{\mathbf{q}}+\text { h.c. }\right] .
$$

Here $\sigma$ is the coupling constant which is determined by the strength of coupling of the hole to the lattice displacement and which does not depend on the mass of isotope. The expression in front of square bracket is the standard Holstein coupling constant $\left.\gamma=\sigma / \sqrt{(} 2 M \Omega_{0}\right)$. Another frequently used coupling constant is $g=\gamma / \Omega_{0}$. The most frequently used measure for the strength of the EPI is the dimensionless coupling constant $\lambda=\gamma^{2} / 4 t \Omega_{0}$.

The reason for the strong EPI in the system described by the $\mathrm{t}-\mathrm{J}$ model is the large energy involved into formation of the Zhang-Rice singlet. This energy of several eVs in the rigid lattice is trivial constant. However, when the lattice is deformed by phonons, change of the amplitudes $t_{p d}$ leads to the strong EPI [61, 82-93]. Estimate for EPI obtained from the three-band model is $\lambda \approx 1$ [62].

Another source of EPI is the Fröhlich interaction with the phonons polarized along $c$ axis which broaden and soften with increase of doping $[94,95]$. These phonons 
recently attracted interest [96] driven by new ARPES data on $\mathrm{Bi}_{2} \mathrm{Sr}_{2} \mathrm{CuO}_{6}$ [97]. However, importance of such phonons was noted long ago [98].

Large EPI in cuprates arises due to strong electronic correlations $[61,89]$ because calculations by Local Density Approximation (LDA) method give the coupling constants which are smaller by an order of magnitude [99]. However, these results are doubtful since the phonon linewidth, obtained by LDA [100], is considerably smaller that the linewidth seen in experiment [101]. The authors of [102] claim that the kink cannot be driven by EPI because effect of EPI on ARPES spectra is negligible. However, as it was shown in [103], calculations by the method used in [102] do not reproduce the linewidth of phonons seen in experiment [104-108]. Hence, conclusions of [102] about kinks are at least doubtful.

\section{Spectroscopy}

There is no adopted agreement whether kinks in ARPES dispersion are driven by interaction with phonons or magnetic resonance mode. However, recent experiments on the isotope effect of the ARPES spectra give confidence that the kinks are due to interaction with phonons. Another evidence for the phonon origin of the kinks follows from the ARPES spectra measurements in the electron doped compounds where kinks and magnetic resonance mode are located on absolutely different energies. The results concerning kinks are presented in Section 3.1.

The most obvious and undebatable evidence for EPI can be found in phonon spectra where EPI is manifested in the softening and broadening of particular phonons. Experimental and theoretical results on the phonon anomalies are reviewed in Section 3.2.

Theoretically, ARPES signal measured in the undoped compounds corresponds to the Lehmann Function (LF) of a hole in the $\mathrm{tt}^{\prime} \mathrm{t}^{\prime \prime}-\mathrm{J}$ model. The LF of this model $[6,37$, 72] has a narrow $\delta$-functional peak at low-energies and high-energy incoherent continuum. Dispersion of the lowenergy peak is in perfect agreement with the experimental momentum dependence. However, even the narrowest width of the experimental peak in the nodal point $(\pi / 2, \pi / 2)$ is larger than the dispersion bandwidth $[57,59]$. This is the main contradiction of the experiment with the $\mathrm{tt}^{\prime} \mathrm{t}^{\prime \prime}-\mathrm{J}$ model because the theoretical width of the peak in the nodal point is zero [37]. The interpretation of the linewidth in terms of EPI [109] was suggested long ago but confronted with the fact that EPI must not only broaden the line but considerably change the dispersion. Solution to the problem of the large linewidth of ARPES line is presented in Section 3.3. For $\lambda>0.4$ in the $\mathrm{t}-\mathrm{J}-\mathrm{H}$ model the agreement with experiment is perfect. At strong EPI the quasiparticle loses its weight, becomes dispersionless, and cannot be seen in ARPES spectra. The whole weight of the quasiparticle is transformed into the broad Franck-Condon shake-off peak whose dispersion inherits the dispersion of the pure t-J model.

The estimate of $\lambda$ was done by several methods. The most detailed and convincing estimates were done in [62] where $\lambda$ was determined from the calculation of the change of Zhang-Rice singlet energy with lattice deformation, from the linewidth of the peak, and the distance of the Franck-Condon peak from the chemical potential. All these methods give $\lambda \sim 1$ which is enough to bring the system into the strong coupling regime. These and other methods to determine $\lambda$ are described in the Section 3.4.

One of the most important experiments to reveal the role of EPI is the isotope effect on the ARPES spectra. Experimentally, the isotope effect on the ARPES of doped compounds was measured many times [110-113] but the most reliable result is obtained in [113]. Keeping in mind how many contradictions were overcome to measure the isotope effect on the doped compounds it is clear why the isotope effect on the undoped compounds, where preparation of specimens is more difficult, was not measured up to now. However, there is a theoretical prediction for the undoped case [46] which is presented in Section 3.5.

It was noted long ago [60] that the net influence of the polaronic effect is unable to explain anomalous temperature dependence of the ARPES spectra. Also, the pure t-J model is unable to explain the experimental data too [114]. However, the joint influence of the magnetic and lattice degrees of freedom, as it is shown in Section 3.6, perfectly explains the anomalous temperature dependence.

One more proof of the interplay of magnetic and lattice effects can be got from the study of the OC of the weakly doped compounds. Two-peak structure of the MIR OC was tacitly resolved in many measurements $[23,27,115-$ 120]. Recent measurement by ellipsometry resolved the twopeak structure clearly [51]. Section 3.7 reviews results of the calculation of the OC in the framework of the t-J-H model [51] where theoretical OC shows two-peak structure due to interplay of magnetic and lattice degrees of freedom. Dependence of the OC on doping gives possibility to estimate dependence of the coupling constant $\lambda$ on doping. It is shown that with $\lambda \sim 1$ in the weakly doped systems the effective EPI decreases with doping coming to the moderate coupling regime $\lambda \sim 0.5$ at the optimal doping.

There are many evidences that the local EPI of the Holstein model cannot explain many features of the hightemperature superconductors. First, it is well known that the very nature of coupling to the Zhang-Rice singlet and Fróhlich coupling to the $c$-axis phonons leads to nonlocal coupling vertexes. Second, the local coupling to phonons leads to huge masses of the holes which are not observed in experiment. One can continue with the fact that the local EPI leads to the contradicting to experiment stability of the antiferromagnetism in doped systems though above motivation is enough to look at the models where EPI is not local. Results concerning nonlocal EPI are presented in Section 3.8.

3.1. Kinks. The nature of the kings is debated since its' discovery. Reasonable explanation of kinks is a battlefield between groups explaining its nature by the interaction with phonons and others prescribing the anomaly to the interaction with the magnetic resonance mode. Typical kinks in ARPES spectra are shown in Figure 1. Early studies [64, 


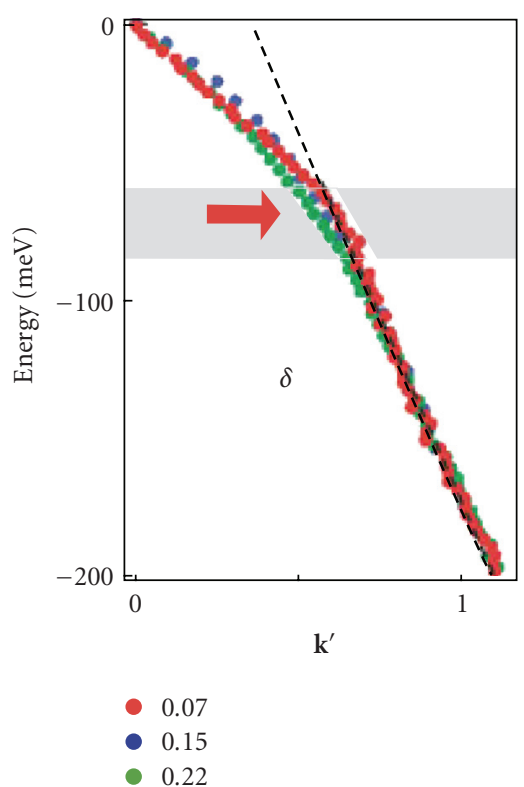

(a)

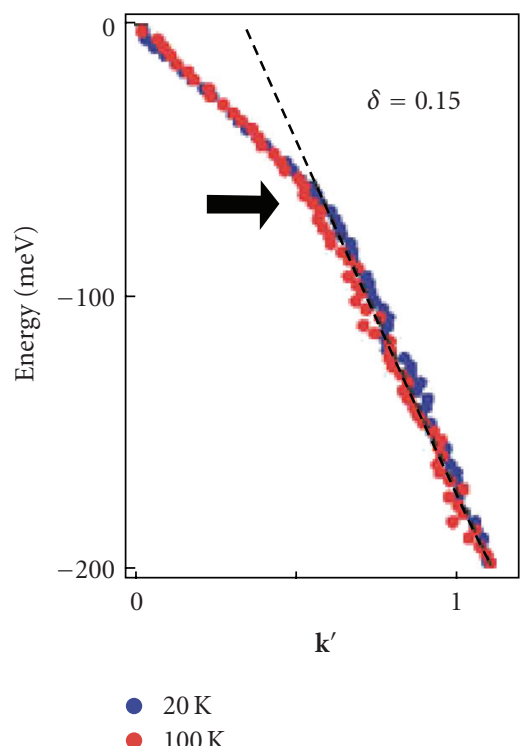

(d)

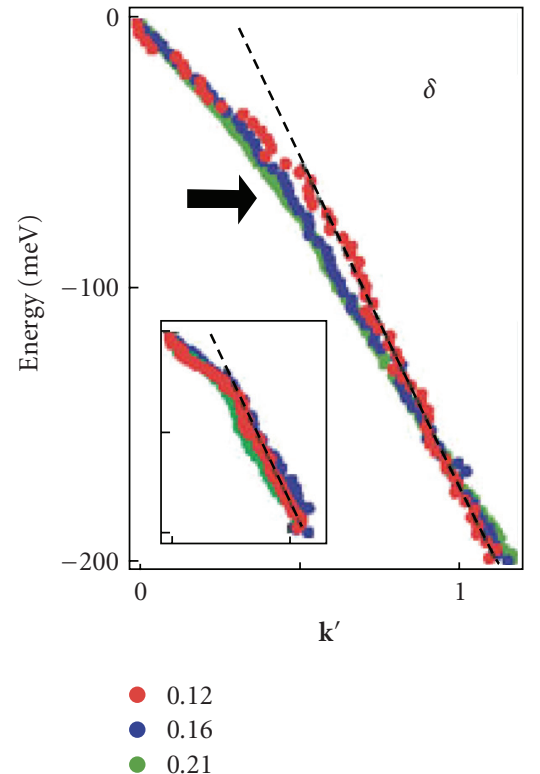

(b)

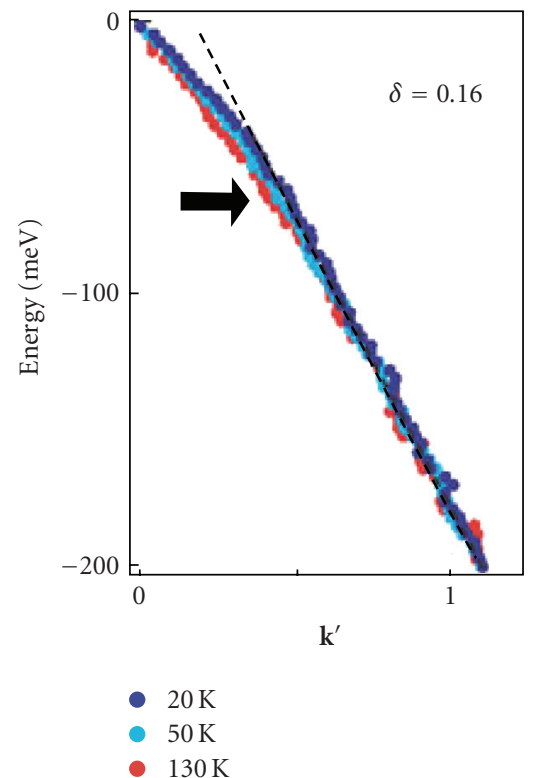

(e)

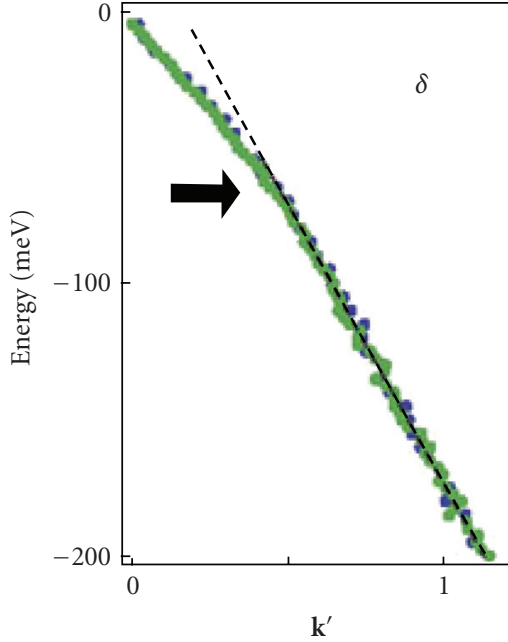

- 0.21

0.24

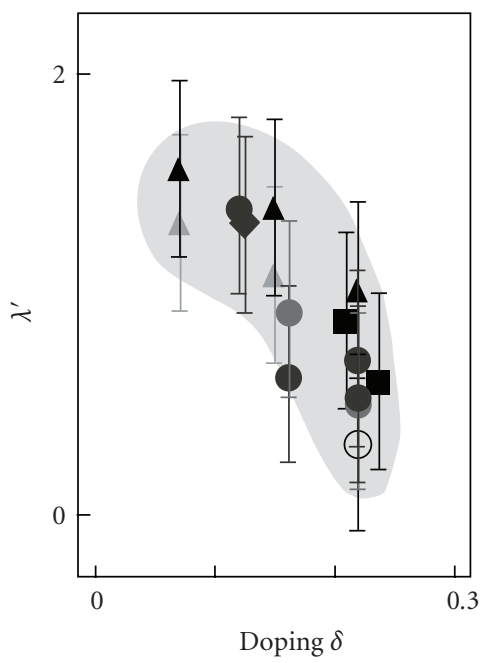

(f)

FIgURE 1: Quasiparticle dispersion in the direction $(0,0)-(\pi, \pi)$ in the compounds $\mathrm{La}_{2-x} \mathrm{Sr}_{x} \mathrm{CuO}_{4}(\mathrm{LSCO}), \mathrm{Bi}_{2} \mathrm{Sr}_{2} \mathrm{CuO} \mathrm{O}_{6}\left(\mathrm{Bi}_{2} 201\right)$, and $\mathrm{Bi}_{2} \mathrm{Sr}_{2} \mathrm{CaCu}_{2} \mathrm{O}_{8}$ (Bi2212) at different temperatures and dopings. Panel (f) shows the value $1+\lambda^{\prime}$, evaluated by the change of the dispersion angle in the weak coupling theory for noninteracting electrons in metal, after Lanzara et al. [64].

121-127] indicated that the anomaly is located on the energy $70 \mathrm{meV}$. Later, refined analysis showed $[97,128]$ that there are structures with smaller energy. Kinks are observed not only along the nodal direction $(0,0)-(\pi, \pi)$ but also in other directions $[125-127,129,130]$.

Kinks were explained by interaction with phonons [90, $91,131,132]$ and by interaction with magnetic resonance mode [129, 133-139] which is observed in the magnetic neutron scattering on the cuprates [140-143]. Actually, it is very difficult to discern theoretically whether kink is governed by magnetic or lattice subsystem. Detailed study [144] has led to conclusion that it is difficult to state which modes give the origin to the kink.

However, the nature of the kink can be revealed by experiments. There are two unambiguous evidences of the phononic nature of the kink. The first proof is obtained in [145]. As it is noted in [145] the optical phonons have energies $40 \mathrm{meV}$ and $70 \mathrm{meV}[146,147]$ while the energy of the magnetic resonance mode in the hole doped compounds is $40 \mathrm{meV}[140,148]$. Therefore, it was very 
difficult to distinguish between phononic and magnetic scenarios because of the similar energy scales of magnetic and phononic excitations. However, recently found magnetic resonance mode in the electron doped compounds [149, $150]$ is on the energy of $10 \mathrm{meV}$ and does not correspond to the energy of the kink whose energy is the same as it was in the hole doped compounds.

Another unambiguous evidence of the phononic nature of the kink follows from the isotope effect on the kink [113]. The change of the ARPES spectra occurs only in the close vicinity of the kink and the characteristic energy of the shift is $3.4 \pm 0.5 \mathrm{meV}$. Theoretical estimate for the isotopic shift of the breathing mode at the energy $70 \mathrm{meV}$ is $3.9 \mathrm{meV}$. This value is in the excellent agreement with the measured isotopic shift.

3.2. Phonons. EPI decreases the frequencies of phonons and leads to decay of the phonons when the line observed in the inelastic neutrons scattering broadens. To determine which of the phonons are anomalous one makes an attempt to fit the experimental phonon branches by some standard, say, shell model. The phonons laying considerably lower than those predicted by the shell model are considered to be anomalous. From this point of view the breathing and half-breathing phonons are anomalous in doped systems [94, 101, 108, 147, 151-156].

Another characteristic feature of the anomalous phonon is the short lifetime manifested in the broad peak with large linewidth which sometimes reaches $5 \mathrm{meV}$ [101] which is an order of magnitude larger than those of another lines whose linewidth is limited by experimental resolution. For example, breathing and half-breathing phonons connected strongly to Zhang-Rice singlet have large linewidth whereas the quadrupole ones which are not coupled to the singlet are narrow [108]. The phonon $O_{Z}^{Z}$ associated with the movement of the oxygen perpendicular to the $\mathrm{CuO}_{2}$ plain has large width of $16 \mathrm{meV}[94,95]$. One more anomalous phonon is $B_{1 g}$ one changing its width with temperature [146, 157159]. There is one more phonon anomaly with wave vector $\mathbf{q}=(0.25,0,0)$ which was believed to be associated with stripe-like inhomogeneities of charge distribution [104] at the doping level $x \approx 1 / 8$. However, the same anomaly is observed at $x \approx 0.08$ [160]. Moreover, it was noticed that the energy and momentum of the kink in ARPES match the corresponding parameters of the phonons [161].

In one of the first theoretical papers considering lattice vibrations in cuprates the phonon softening was found by exact diagonalization technique in the model where effective Hamiltonian was obtained from the three-band model [162]. Softening of the breathing $\mathbf{q}=(\pi, \pi)$ and halfbreathing $\mathbf{q}=(\pi, 0)$ phonons was calculated in $[83,84,87$, 92]. The calculation predicted dependence of the softening on the doping concentration which was later confirmed experimentally [95]. Also, theoretical data on the phonon broadening [84] showed, in agreement with experiment, that the peak width is maximal at $\mathbf{q}=(\pi, 0)$ and much smaller at $\mathbf{q}=(\pi, \pi)$.

The authors of the paper [88] studied the dependence of the EPI matrix element on the wave vectors of the electron

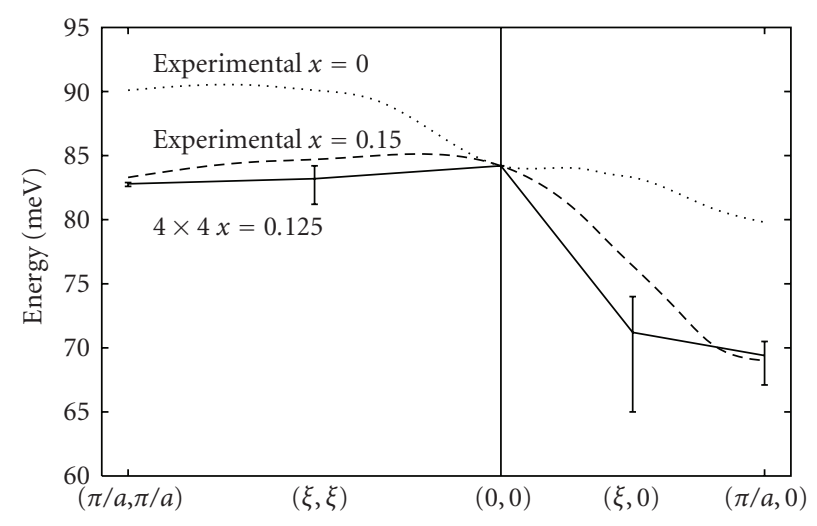

Figure 2: Phonon dispersion in the $(1,0)$ and $(1,1)$ directions. Experimental results are shown by dotted lines for $x=0$ and $x=0.15$. Theoretical results (full curve) are shown for $x=0.125$. The bars in theoretical results show the spread due to different boundary conditions, after Rösch and Gunnarsson [61].

and phonon and concluded that the interaction is strongly anisotropic. The authors found that the maximum of the broadening of the longitudinal optical phonons is associated with effective coupling of the $d$-symmetry pairing. Papers $[163,164]$ considered the charge response of the hightemperature superconductors arising from the ionic nature of the compounds. These papers predicted strong softening of the phonons with displacement along $c$-axis prior to experiment.

Using the first principle calculations and three-band model the paper [61] introduces the t-J-H model with derived from the first principles parameters. The exact diagonalization used to obtain the phonon spectral function gives the phonon dispersion which is in a good agreement with experimental data (see Figure 2) at the doping concentration $x \approx 0.125$.

3.3. Ghost Particles in the ARPES. The spectral function of the $\mathrm{t}-\mathrm{J}-\mathrm{H}$ model (3)-(7) was previously calculated by exact diagonalization (ED) of small clusters [165] and in the Non-Crossing Approximation (NCA) where all crossings of propagators are neglected $[166,167]$. However, small size of the clusters in ED gives essentially discrete spectrum and prevent from studying of the lineshape $[165,168]$. On the other hand the NCA is valid, strictly speaking, only for the weak coupling to phonons [42]. The NCA is good for magnons since the spin $1 / 2$ cannot be flipped more than one time and, hence, the multiple accumulation of the bosons on one site is impossible. However, there is an accumulation of multiple bosons in the EPI channel and, hence, one has to avoid NCA for correct treatment of the EPI.

The implementation of the DMC method in [42] takes into account the mutual crossing of the phonon propagators but neglects both crossing of the phonon and magnon lines and mutual crossing of magnon lines. The NCA in the pure $\mathrm{t}-\mathrm{J}$ model, as it was shown by comparison of the NCA results with the ED data $[67,68,71,169,170]$, is good for $J / t \leq$ 0.4. Similar conclusion is drawn for the t-J-H model [171]. Also, recent calculations of the spectral function of the $\mathrm{t}-\mathrm{J}-\mathrm{H}$ 
model [172] by variational method [173, 174], which does not use NCA, confirmed the approximation made in [42].

Figures 3(a)-3(e) show the low-energy part of the spectral function in the ground state at $\mathbf{k}=(\pi / 2, \pi / 2)$ in the regime of weak, intermediate and strong EPI. $\lambda$-dependence of the energies of the peaks (see Figure 3(f)), $Z^{\mathrm{k}=(\pi / 2, \pi / 2)}$ factor of the lowest peak (see Figure $3(\mathrm{~g})$ ), and average number of phonons in the phonon cloud (see Figure 3(h)) is typical for the self-trapping phenomenon $[40,175,176]$.

Momentum dependence of the spectral function is shown in Figures 4(a)-4(d). Energy of the lowest peak with small spectral weight (shown by vertical arrows in Figures $4(\mathrm{a})-4(\mathrm{~d})$ ) does not demonstrate any visible momentum dependence. To the contrary, dispersion of the broad peak is perfectly reproduced by the relation (see Figure $4(\mathrm{e})$ )

$$
\begin{aligned}
\varepsilon_{\mathbf{k}}= & \varepsilon_{\min }+\frac{W_{J / t}}{5} \\
& \times\left\{\left[\cos k_{x}+\cos k_{y}\right]^{2}+\frac{\left[\cos \left(k_{x}+k_{y}\right)+\cos \left(k_{x}-k_{y}\right)\right]^{2}}{4}\right\},
\end{aligned}
$$

which perfectly describes the dispersion of the pure $\mathrm{t}-\mathrm{J}$ model in the wide range of parameters $0.1<J / t<0.9$ [71]. Note that such behavior of the broad Franck-Condon shake-off peak is robust for the whole strong coupling regime of the EPI.

At low temperature the spectral function in the adiabatic approximation is the sum of transitions between the lower $E_{\text {low }}(Q)$ and the upper $E_{\text {up }}(Q)$ sheet of the adiabatic potential. The transitions are weighted by the wave function of the lower sheet $\left|\psi_{\text {low }}(Q)\right|^{2}$ [58]. If EPI is absent both in the initial $E_{\text {low }}(Q)=Q^{2} / 2$ and final $E_{\text {up }}(Q)=\mathscr{D}+Q^{2} / 2$ states of transition, the spectral peak has maximum at the energy $\mathcal{D}$. However, if the EPI $\Delta E_{\text {up }}(Q)=-\lambda Q$ is present only in the final state $\Delta E_{\mathrm{up}}(Q)=-\lambda Q$, the upper sheet of the adiabatic potential $E_{\text {up }}(Q)=\mathscr{D}-\lambda^{2} / 2+(Q-\lambda)^{2}$ has the same energy at $Q=0$. Hence, since the maximum of the probability in the initial state $\left|\psi_{\text {low }}(Q)\right|^{2}$ is at $Q=0$, the energy of the peak of the spectral function of the transition is still at the same energy $D$ (see Figure $4(\mathrm{f})$ ). Note that the situation described above is the same as in the ARPES experiment in undoped cuprates. There is no EPI in the half-filled system (initial state) but there is EPI in the system with hole (final state).

The lowest dispersionless peak with small weight is too small to be easily observed in experiment. To the contrary one can easily observe wide Franck-Condon shake-off peak which mimics the dispersion of the pure $t-J$ model. This theoretical picture suggests that the chemical potential of the weakly doped cuprates should not be associated with the broad peak but must be pinned by invisible real quasiparticle. The above conclusion was lately confirmed in experiment [59] (see Figure 5). Chemical potential is pinned to the peak $B$ while the energy of the broad peak $A$ is far from the peak B.

3.4. The Values of $\lambda$. One of the most detailed study of the strength of the EPI was undertaken by several methods in
[62]. For the explanation of the large linewidth of the ARPES spectra in the undoped $\mathrm{La}_{2} \mathrm{CuO}_{4}$ the authors of [62] use the Hamiltonian obtained from the reduction of the three-band model. The parameters of the model were such that they give correct description of the phonon spectra in the realistic model of the $\mathrm{La}_{2} \mathrm{CuO}_{4}$ with 21 phonon branches [61]. The EPI in the realistic model with 21 phonon branches reads

$$
H_{\mathrm{ep}}=\frac{1}{\sqrt{N}} \sum_{\mathbf{q} \gamma \mathbf{i}} g_{\mathbf{q}^{\nu}}\left(1-n_{\mathbf{i}}\right) \sqrt{2 \omega_{\mathbf{q}} v} Q_{\mathbf{q} v} e^{i \mathbf{q}} .
$$

This Hamiltonian describes the interaction with the empty places forming singlet in the t-J model. Interaction is linear with respect to the lattice coordinates $Q_{\mathbf{q}}$ and coupling constants $g_{\mathbf{q} v}$. The phonon mode of frequency $\omega_{\mathbf{q} v}$ is defined by its wave vector $\mathbf{q}$ and phonon branch index $\nu . n_{\mathrm{i}}$ is the electron filling of the site $\mathbf{R}_{\mathbf{i}}$ and the total number of sites is $N$.

Effective EPI constant is defined in [62] as

$$
\lambda \equiv \frac{1}{N} \sum_{\mathbf{q} v} \frac{\left|g_{\mathbf{q} v}\right|^{2}}{4 t \omega_{\mathbf{q} v}} .
$$

The value of $\lambda$ at $t=0.4 \mathrm{eV}$ is estimated as $\lambda=1.2$. Authors of [62] calculated the differential spectral distribution of the effective EPI

$$
\gamma(\omega)=\frac{1}{N} \sum_{\mathbf{q} v} \frac{\left|g_{\mathbf{q} v}\right|^{2}}{\omega_{\mathbf{q} v}} \delta\left(\omega-\omega_{\mathbf{q} v}\right) .
$$

The spectral distribution (11) is compared with the fine structure of renormalization of the dispersion in the vicinity of kink measured in [128]. Good agreement of these two data encourages to believe into the phononic nature of the kinks and ensures that the estimate for the effective $\lambda$ is correct.

To estimate the value of effective EPI from the difference of the energy of the real quasiparticle and Franck-Condon peak authors of [62] introduced the scaling $g_{\mathbf{q} v} \rightarrow \Lambda g_{\mathbf{q} v}$ of the coupling constant in the (10). The theoretical binding energy for $\Lambda=1$ is $1.2 \mathrm{eV}$ which is considerably larger than the experimental value $0.5 \mathrm{eV}$. The binding energy is proportional to $\Lambda^{2}$ and even small overestimate of the coupling constants, originating from the underestimate of the screening in the shell model, can lead to strong overestimate of the binding energy. Reasonable values of the binding energy and linewidth can be obtained for $\Lambda=0.8$ which corresponds to $\lambda=0.75$. Thus, one can conclude that EPI in the undoped cuprates ranges like $0.75<\lambda<1.2$. These values are larger than $\lambda_{c}=0.6$ [46] which is required for transition of the $\mathrm{tt}^{\prime} \mathrm{t}^{\prime \prime}-\mathrm{J}-\mathrm{H}$ model into the strong coupling regime.

Another source of the information about the strength of the EPI is the temperature dependence of the ARPES spectra. Comparison of the experimentally measured temperature dependence [177] and that calculated from the t-J-H model [50] gives the estimate $0.5<\lambda<1.0$. Comparison of the experimental and theoretical data for high-energy part of the ARPES spectra gives the same values. The 


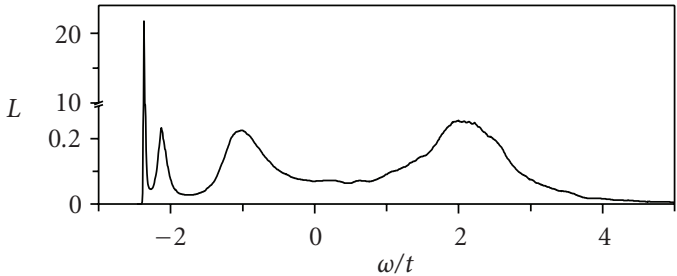

(a)

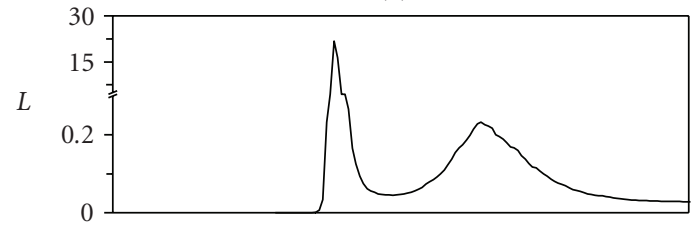

(b)

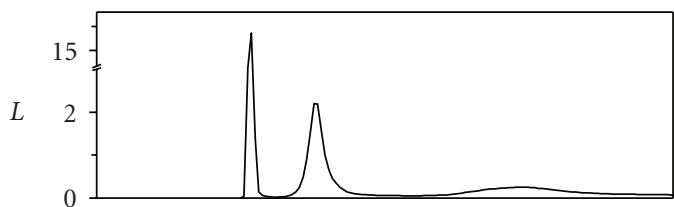

(c)

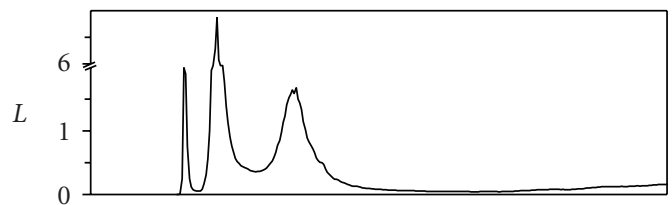

(d)

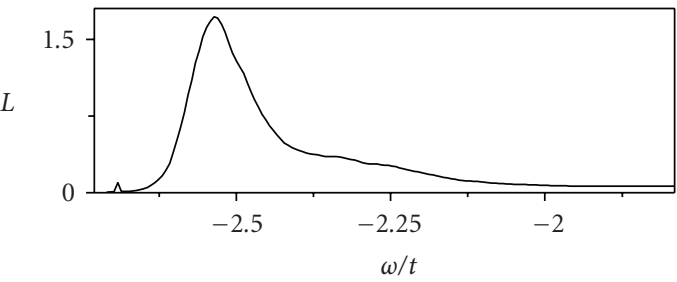

(e)

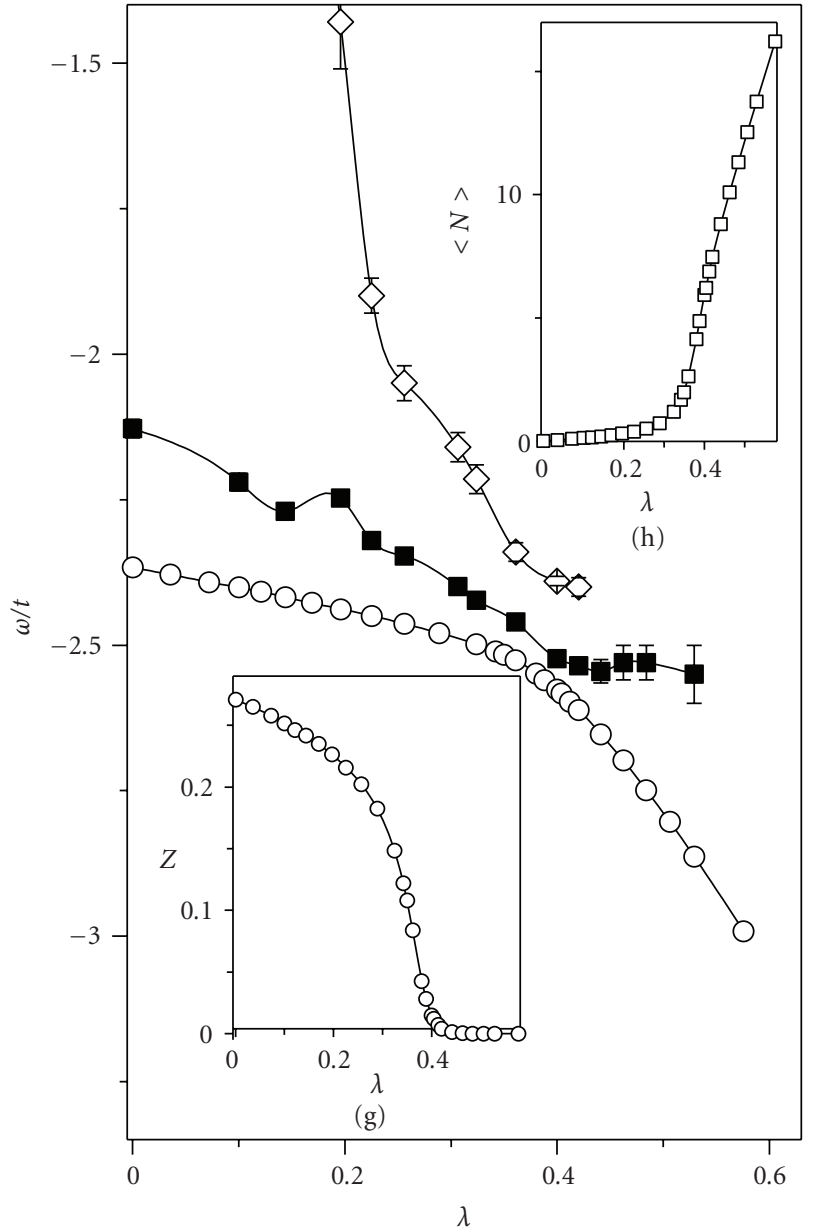

(f)

Figure 3: (a) Lehmann spectral function $\mathrm{L}$ of the ground state at $\mathbf{k}=(\pi / 2, \pi, 2)$ for $J / t=0.3$ and $\lambda=0$. Low-energy spectral function $\mathrm{L}$ in the ground state $\mathbf{k}=(\pi / 2, \pi, 2)$ at $J / t=0.3$ : (b) $\lambda=0$; (c) $\lambda=0.3$; (d) $\lambda=0.4$; (e) $\lambda=0.46$. $\lambda$-dependence at $J / t=0.3$ : (f) energies of the lowest resonances; (g) $Z$-factor of the lowest resonance; (h) average number of phonons.

waterfalls, observed in the range 1 to $2 \mathrm{meV}$ [178-182], were reproduced by artificial broadening of the string resonances of the t-J model $[183,184]$. Consecutive search for the physical mechanism for the artificial broadening led to conclusion that the broadening caused solely by nonzero temperature is not enough to explain the linewidth and has to take into account rather considerable EPI [63] with $\lambda=$ 0.65 .

Another method to get the value of $\lambda$ is to compare the theoretical growth of the linewidth when the energy of quasiparticle exceeds the frequency of the dispersionless phonon with that measured above the kink energy. Such analysis for experimentally measured ARPES spectra in the electron doped compounds $\mathrm{Sm}_{2-x} \mathrm{Ce}_{x} \mathrm{CuO}_{4}(x=0.1,0.15,0.18)$, $\mathrm{Ng}_{2-x} \mathrm{Ce}_{x} \mathrm{CuO}_{4}(x=0.15)$, and $\mathrm{Eu}_{2-x} \mathrm{Ce}_{x} \mathrm{CuO}_{4}$ [145] gives the estimate $\lambda=0.8$.
Recently developed method of time resolved electron diffraction is capable of studying the time evolution of the excited states [185]. Comparison of the experimental data with the results obtained in the framework of the theory of relaxation in metals [186] led to conclusion that anisotropic $\lambda$ is in the range $0.08<\lambda<0.55$. And last, comparison of the isotope shift of the OC of the undoped compounds [187] with theoretical results [188] gave the estimate $\lambda \geq 1$ [189].

3.5. Isotope Effect in ARPES. Experimentally, isotope effect (IE) was studied only for highly doped compounds and the main emphasis was made on studies of the kinks. The ARPES spectra data were measured in compounds where oxygen was represented by ${ }^{16} \mathrm{O}$ and ${ }^{18} \mathrm{O}$ isotopes. The first results for IE on the kinks in ARPES spectra $[110,190]$ received a lot of skepticism caused by the structure of the isotope shift. 


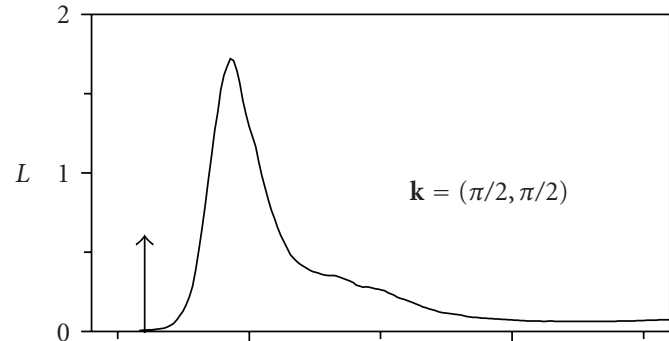

(a)

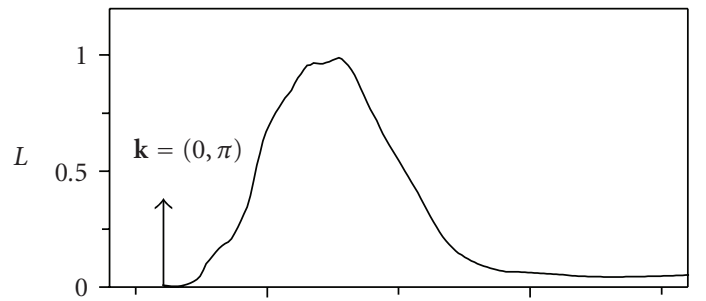

(b)

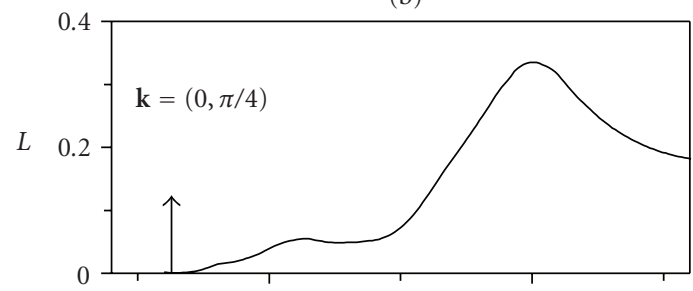

(c)

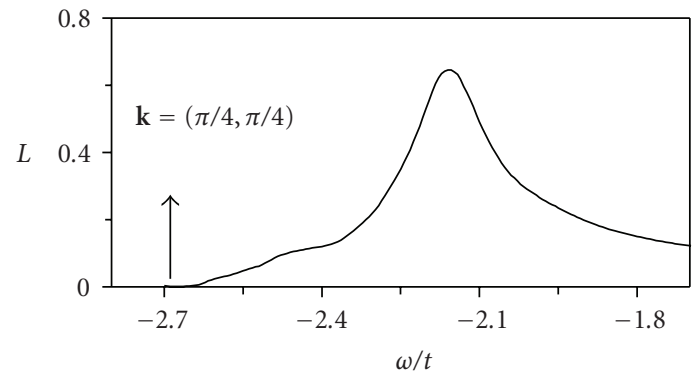

(d)

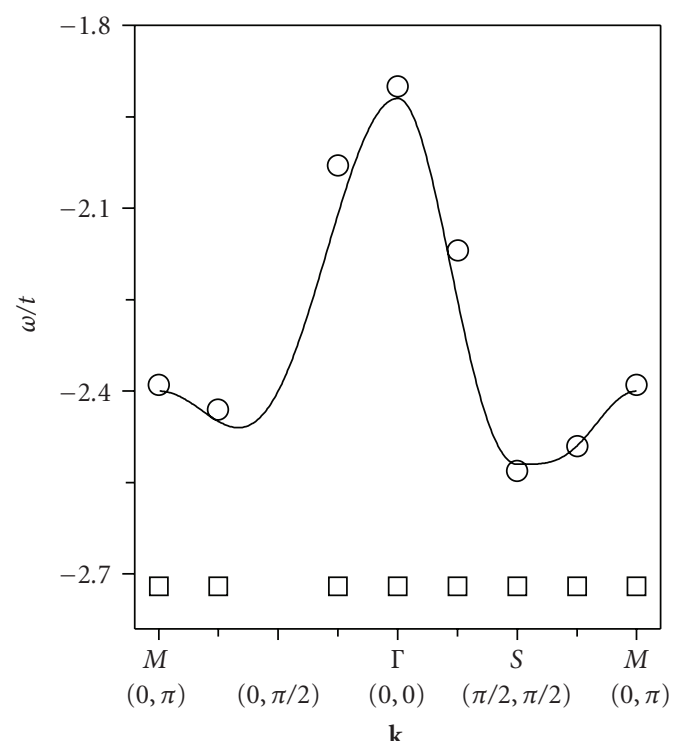

(e)

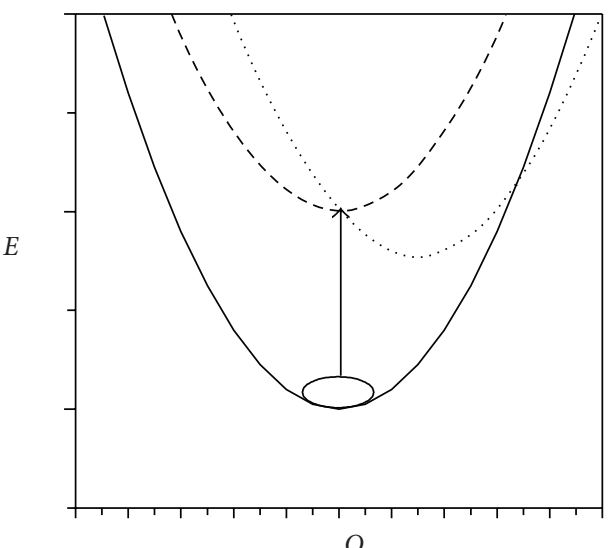

(f)

FIgure 4: Low-energy part of the spectral function of the hole at $J / t=0.3$ and $\lambda=0.46$ (a)-(d). The vertical arrows in (a)-(d) denote the position of the quasiparticle invisible in the scale of the figure because of low weight. (e) Dispersion of resonances at $J / t=0.3$ : broad resonance (circles) and the quasiparticle with small weight (squares) at $\lambda=0.46$. The solid line is the dispersion of the hole (8) in the pure $\mathrm{t}-\mathrm{J}$ model at $J / t=0.3\left(W_{J / t=0.3}=0.6\right)$ and $\varepsilon_{\min }=-2.52$. Panel (f) shows the potential of ground state $Q^{2} / 2$ (solid line), potential of the excited state without the lattice relaxation (dashed line), and potential of the excited state after the lattice relaxation $D+(Q-\lambda)^{2} / 2-\lambda^{2} / 2($ dotted line).

The shift was observed in the high-energy region beyond the phonon energy.

Further experiments were in contradiction with the results obtained in $[110,190]$. Measurements [111] did not observe the IE at large energies. It was also noted that even tiny sample misalignment of 0.1 degree can lead to considerable shifts at large energies. Also, measurements presented in [111] noted the general shift of the spectral features by $3 \mathrm{meV}$ which is consistent with results of tunneling experiments [191, 192].

The most detailed and precise analysis of the IE on ARPES spectra was presented in [113]. IE on ARPES was observed only in the vicinity of the kink and the shift of spectral feature around $3.4 \pm 0.5 \mathrm{meV}$ (see Figure 6) was found. Extremely high precision of the measurement done in [113] arises from the novel method of low-energy ARPES with resolution much higher [193] than that typical for conventional ARPES technique. Calculations show that the breathing mode at the energy $70 \mathrm{meV}$ has to be shifted by the IE by $3.9 \mathrm{meV}$ which is in perfect agreement with experimental data.

There are a lot of studies of the IE on the polaronic systems. Holstein model was studied in [194-197] by the path integral Monte Carlo method [198] and Dynamical Mean Field Theory (DMFT) method [199]. The change of the effective mass by the isotope substitution is the largest 


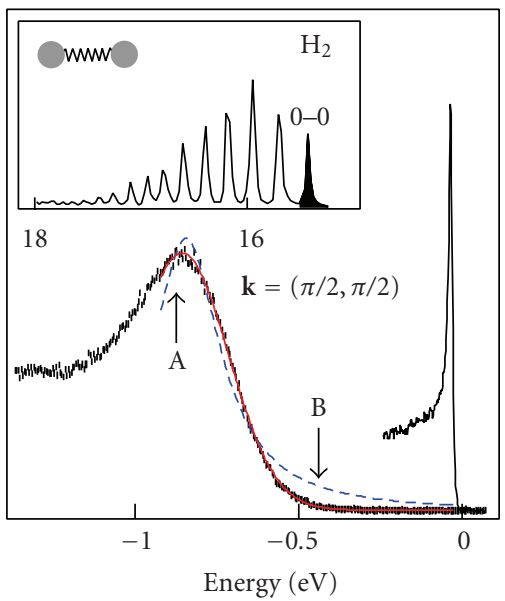

(a)

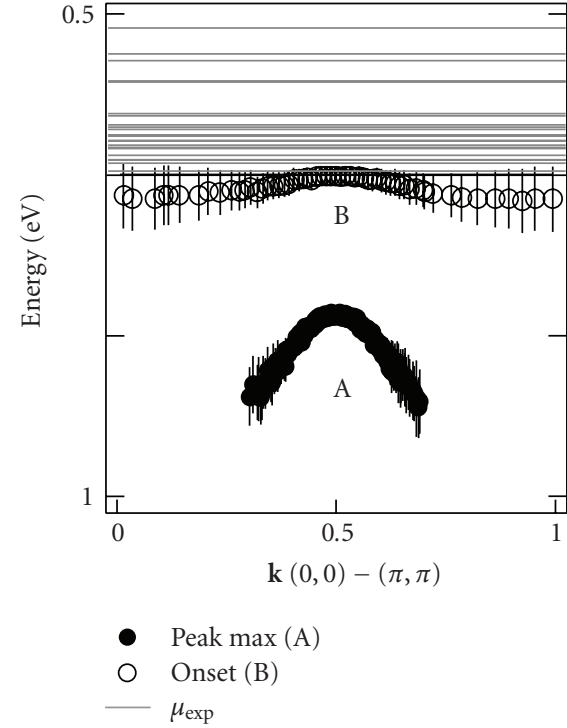

(b)

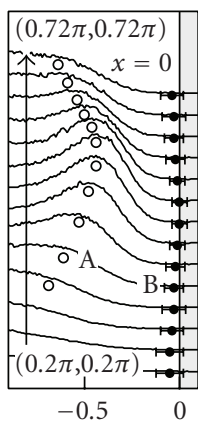

(c)

FIgURE 5: (a) ARPES spectrum of the undoped $\mathrm{Ca}_{2} \mathrm{CuO}_{2} \mathrm{Cl}_{2}$ in the nodal point $\mathbf{k}=(\pi / 2, \pi / 2)$ fitted by Lorentzian (dashed line) and Gaussian (thick solid line). The notations A and B define the maximum of the peak and the point where spectral density arises, respectively. Narrow peak in the vicinity of zero energy is the ARPES spectrum of $\mathrm{SrRuO}_{4}$. (b) Dispersion of A and B. The positions of the chemical potentials for different samples are shown by the horizontal lines. (c) Experimental ARPES spectra in the vicinity of the nodal point, after Shen et al. [59].

in the intermediate coupling regime. The IE in the HolsteinHubbard systems was studied in [200-202].

The IE on the ARPES spectra for three-band model was studied in [132]. The interplay of EPI and electronic correlation is neglected in this paper but, however, the usage of the realistic model leads to many new and interesting conclusions. In agreement with experiment [113] the maximal IE is observed in the vicinity of the phonon frequency. It was also concluded that the kink cannot be caused by the electronic subsystem alone.

Nowadays it is important to attempt experiments to study IE on the ARPES spectra of undoped compounds. In addition to the high-temperature superconductors, there are other systems where broadening of the ARPES peak by EPI can be considered as a possible scenario. The list of such systems includes diatomic molecules [203], manganites with colossal magnetoresistance [204], quasi-one-dimensional Peierls conductors [205, 206], and Verwey magnetites [207].

Theoretical study of the IE on ARPES spectra of undoped system, specific for high-temperature superconductors because of choice of the $\mathrm{tt}^{\prime} \mathrm{t}^{\prime \prime}-\mathrm{J}-\mathrm{H}$ model, was done in [46]. Dimensionless coupling constant $\lambda=\gamma^{2} / 4 t \Omega$ does not depend on the isotope mass in case when the relation between phonon frequency and mass $\Omega \sim 1 / \sqrt{M}$ holds. The isotopic factor is defined as $\kappa_{\text {iso }}=\Omega / \Omega_{0}=\sqrt{M_{0} / M}$. Parameters for $\mathrm{tt}^{\prime} \mathrm{t}^{\prime \prime}-\mathrm{J}-\mathrm{H}$ model were chosen to reproduce the experimental ARPES spectra dispersion [6]: $J / t=0.4, t^{\prime} / t=-0.34$, and $t^{\prime \prime} / t=0.23$. Phonon frequency [5] is $\Omega_{0} / t=0.2$ and the isotopic factor for change from ${ }^{16} \mathrm{O}$ to ${ }^{18} \mathrm{O}$ is $\kappa_{\text {iso }}=\sqrt{16 / 18}$.

To avoid errors the ARPES spectra in [46] were calculated for nonsubstituted compound $\left(\kappa_{\text {nor }}=1\right)$, actual isotope substituted compound $\left(\kappa_{\text {iso }}=\sqrt{16 / 18}\right)$, and imaginary antiisotope substituted compound $\left(\kappa_{\mathrm{ant}}=\sqrt{18 / 16}\right)$. Monotonic dependence of the spectral function and other parameters on $\kappa$ ensures in the absence of instability. The errorbar of a number $\mathcal{A}$ can be evaluated using values $\mathcal{A}_{\text {iso }}-\mathcal{A}_{\text {nor }}$, $\mathcal{A}_{\text {nor }}-\mathcal{A}_{\text {ant }}$, and $\left(\mathcal{A}_{\text {iso }}-\mathcal{A}_{\text {ant }}\right) / 2$.

Figure 7 shows the IE on the low-energy part of ARPES spectra for different EPI couplings in the nodal and antinodal points. All spectral features are shifted to larger energies for larger mass $(\kappa<1)$. The shift of the broad Franck-Condon peak is considerably larger than the shift of the narrow peak corresponding to the real quasiparticle. Moreover, for larger values of $\lambda$ the shift of quasiparticle energy goes to zero and the only influence of the IE is the decrease of the spectral weight $Z$ with the increase of the isotope mass. To the contrary, shift of the Franck-Condon peak is not suppressed by the increase of EPI. In all cases, except the spectral function in the nodal point at $\lambda=0.62$ (see Figures 7 (a) and $7(\mathrm{~b}))$ where weight $Z$ of the quasiparticle is still large, there is general feature of the IE on the broad Franck-Condon peak. The height of this peak increases with increase of the isotope mass. Taking into account the sum rule $\int_{-\infty}^{+\infty} L_{\mathbf{k}}(\omega)=1$, very small weight $Z$ of the quasiparticle peak, and the complete absence of IE on the hight energy spectrum [46] one can conclude that the Franck-Condon peak decreases its width with the increase of the isotope mass.

The spectral function of the independent oscillator model is the Poisson distribution [208]:

$$
L(\omega)=\exp \left[-\frac{\xi_{0}}{\kappa}\right] \sum_{l=0}^{\infty} \frac{\left[\xi_{0} / \kappa\right]^{l}}{l !} g_{\kappa, l}(\omega) .
$$




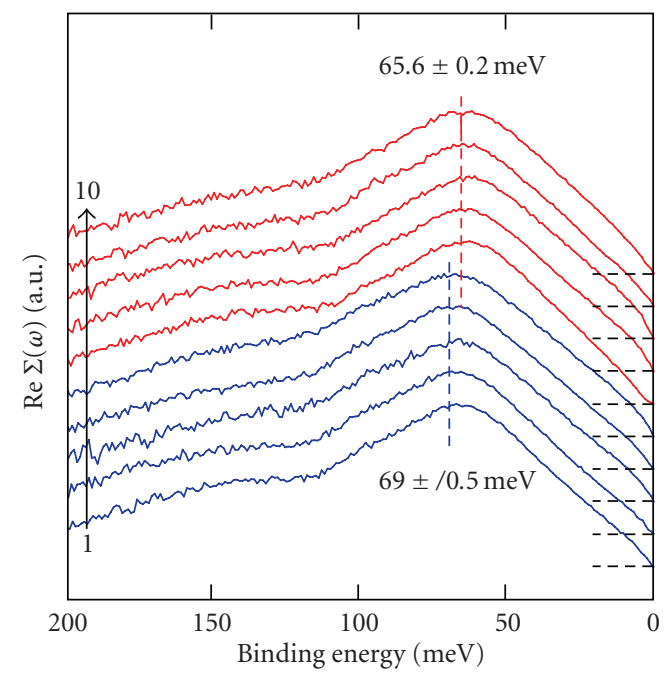

(a)

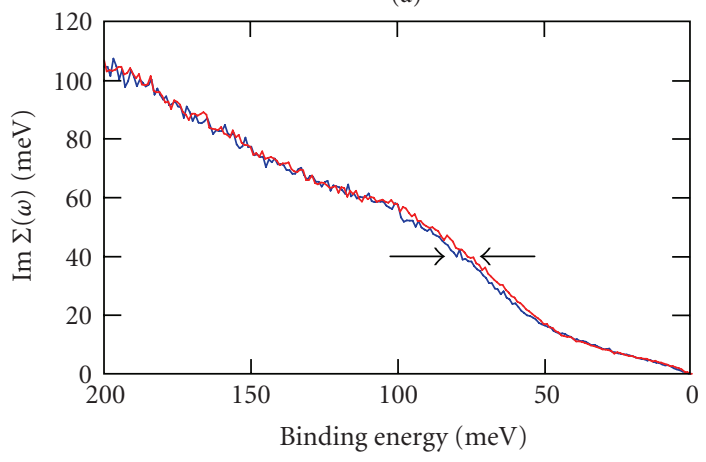

(b)

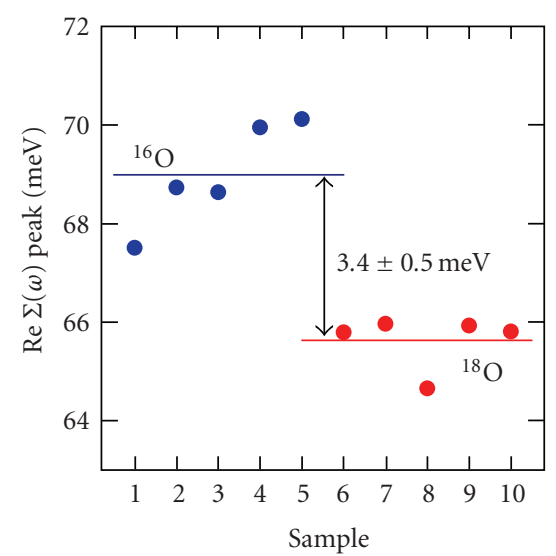

(c)

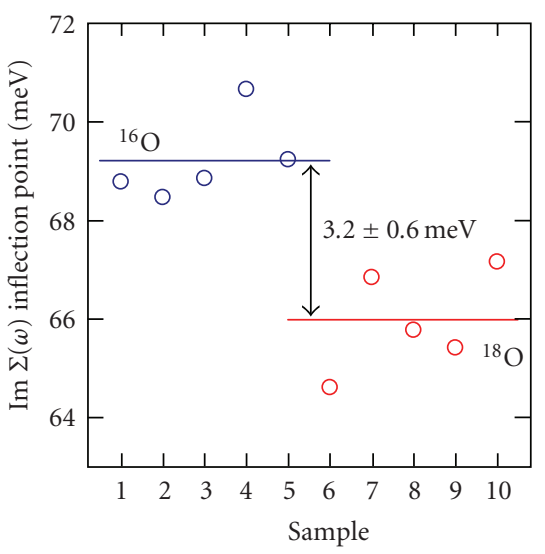

(d)

$$
-{ }^{16} \mathrm{O}
$$

Figure 6: (a) Real part of the self-energy $\operatorname{Re} \Sigma(\omega)$ from five samples both for ${ }^{16} O$ (lower curves) and ${ }^{18} O$ (upper curves). All $\operatorname{Re} \Sigma(\omega)$ are deduced by subtracting a bare band dispersion from the experimental one. (b) Imaginary part of the self-energy $\operatorname{Im} \Sigma(\omega)$ determined from the full width of the momentum distribution curves. (c), (d) Obtained kink energy as a function of sample numbers both for ${ }^{16} \mathrm{O}$ (to the left) and ${ }^{18} \mathrm{O}$ (to the right) from $\operatorname{Re} \Sigma(\omega)$ and $\operatorname{Im} \Sigma(\omega)$, after Iwasawa et al. [113].

Here $\xi_{0}=\gamma_{0}^{2} / \Omega_{0}^{2}=4 t \lambda / \Omega_{0}$ is dimensionless coupling constant for initial system, and $g_{\kappa, l}(\omega)=\delta\left[\omega+4 t \lambda-\Omega_{0} \kappa l\right]$ is a $\delta$-function. For strong EPI limit the exactly solvable independent oscillator model is very helpful to describe the IE on ARPES spectra because the most of properties of the numeric solution are very close to the properties of the spectrum described (12).

3.6. Temperature Dependence of ARPES. The unique feature of the undoped and underdoped compounds with hightemperature superconductivity is the strong interplay of the lattice and magnetic degrees of freedom. One of the most vivid consequence of this interplay is the anomalous temperature dependence of the ARPES spectra. Really, it was realized long ago that the very scale of the experimental temperature dependence is considerably larger than that predicted by the conventional polaron theory [60]. The magnetic subsystem alone is also a wrong candidate [114] to describe the experimental anomaly since the typical energy scale of the magnon energy is $\sim 2 J \approx 0.2 \mathrm{eV}$ which is even larger then the typical phonon energies $\sim \Omega_{0} \approx 0.04 \mathrm{eV}$. Recent studies revealed one more puzzling feature questioning the polaronic scenario in general. The temperature dependence of the width of the broad FranckCondon peak is linear in the range $200 \mathrm{~K}<T<400 \mathrm{~K}$ [177] and extrapolates to zero at zero temperature.

Previously, the ARPES spectra of the $\mathrm{t}-\mathrm{J}-\mathrm{H}$ model were studied by exact diagonalization method $[165,209,210]$, in the noncrossing approximation $[166,167]$, and by DMC method $[42,46]$. All these calculations were done for the case of zero temperature. All the above methods have their own difficulties preventing their reliable and fast generalization to finite temperatures.

A novel method capable of obtaining reliable data on the ARPES spectra of the $\mathrm{t}-\mathrm{J}-\mathrm{H}$ model at finite temperature was developed in $[50,211]$. The main difficulty for studying of the $\mathrm{t}-\mathrm{J}-\mathrm{H}$ model lies in the profound difference between interactions of a hole with phonons and magnons. The 


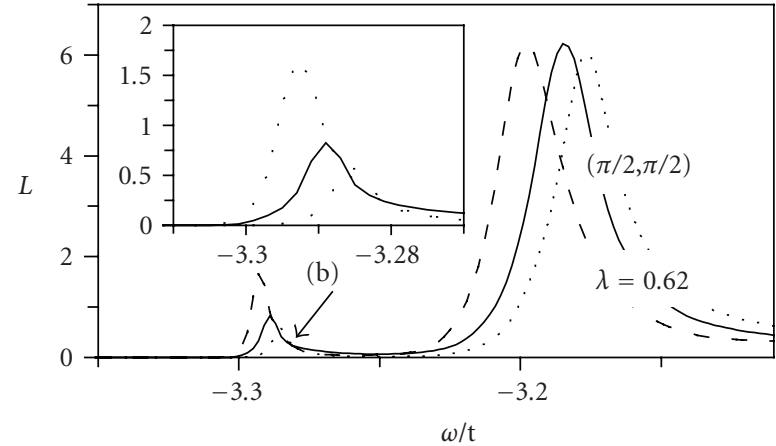

(a)

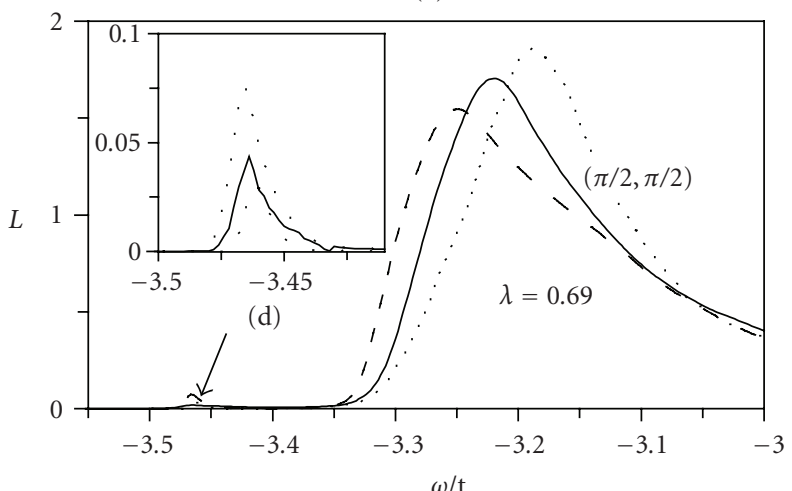

(c)

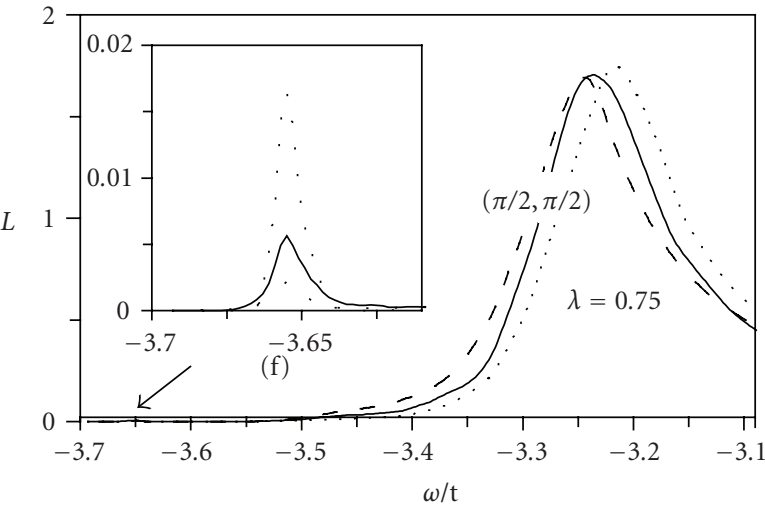

(e)

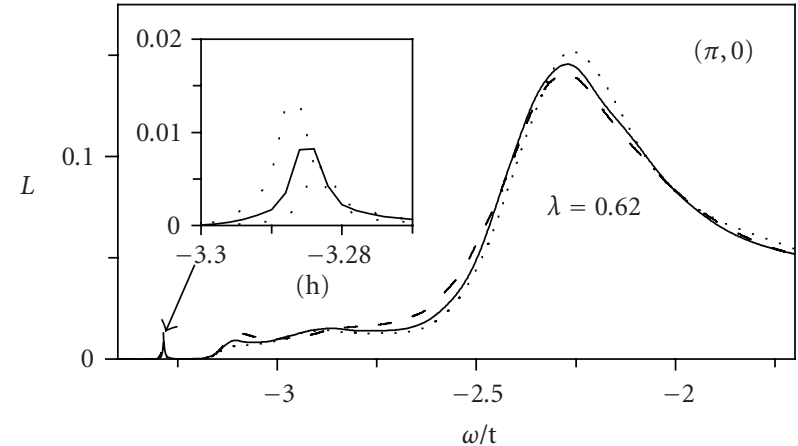

(g)

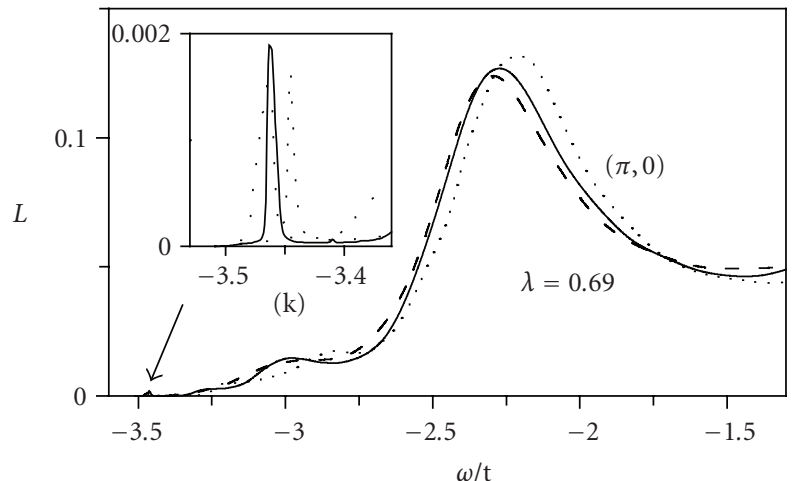

(i)

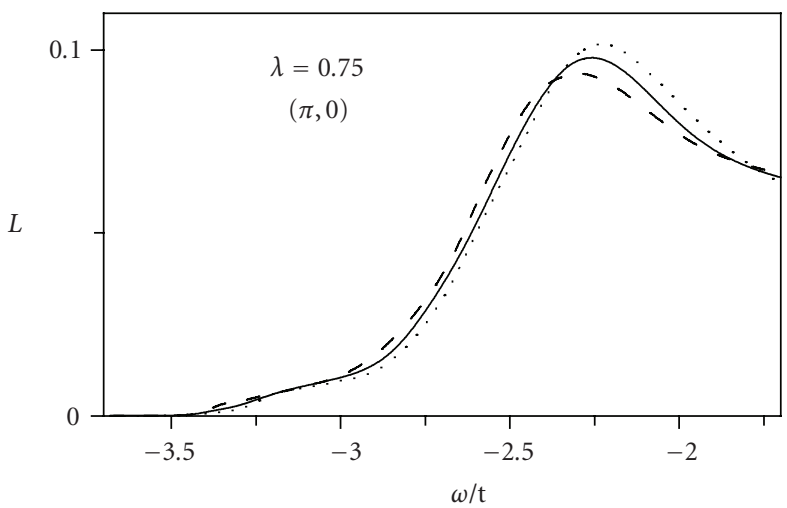

(l)

FIGURE 7: Low-energy part of the spectral function. Spectra of normal, isotope substituted, and "anti-isotope" substituted compounds are drawn by solid, dotted, and dashed lines, respectively. (a), (c), (e) and (g), (i), (l) correspond to nodal $(\pi / 2, \pi / 2)$ and antinodal $(\pi, 0)$ points. Insets (b), (d), (f), (h), and (k) show the peak corresponding to real quasiparticle.

Hybrid Dynamical Momentum Average (HDMA) method, developed in [50], just using difference of two interactions treating them with different techniques. The HDMA method unites advantages of classical Momentum Average (MA) method [212-221], which keeps information on the dispersion of the bare quasiparticle, and self-consistent Dynamical Mean Field Theory approximation (DMFT) [222-232], which is capable of treating strong but local interactions nonperturbatively.

Although the energy scales of bosons and phonons are basically the same, the nature of interactions of the hole with magnons and phonons is considerably different. The interaction with magnons is essentially momentum dependent and always weak. Really, spin $1 / 2$ cannot be flipped more than once limiting, thus, the maximal number of magnons on one site to one [233]. Hence, for small enough values of $J / t[68,171] \mathrm{NCA}$ is a good approximation. To the contrary, interaction with phonons is local and can be very strong. Thus, one cannot use NCA for phonons since it fails already for intermediate couplings [42]. Hence, one is to treat magnons by a weak coupling method which is able to handle the momentum dependence carefully and sum the phonon variables by a local nonperturbative method.

Nonperturbative approaches which neglect the momentum dependence of self energy on momentum are MA 
and DMFT methods. In both cases, the hole self-energy is expressed in terms of continuous fraction:

$$
\Sigma_{\mathrm{h}-\mathrm{ph}}[\alpha(\omega)]=\frac{\gamma^{2} \alpha\left(\omega-\Omega_{0}\right)}{1-\frac{2 \gamma^{2} \alpha\left(\omega-\omega_{0}\right) \alpha\left(\omega-2 \Omega_{0}\right)}{1-\frac{3 \gamma^{2} \alpha\left(\omega-2 \Omega_{0}\right) \alpha\left(\omega-3 \Omega_{0}\right)}{1-\cdots}}}
$$

Then, DMFT and MA differ in the definition of the function $\alpha(\omega)$ which is determined from the self-consistent procedure in DMFT and obtained from the momentum average of the Green function in MA. Suggested in [50] approach introduces the self-energy as a sum of contribution form magnetic and phononic subsystems:

$$
\Sigma_{\mathrm{tJH}}(\mathbf{k}, \omega)=\Sigma_{\mathrm{h}-\mathrm{mag}}^{\mathrm{SCBA}}(\mathbf{k}, \omega)+\Sigma_{\mathrm{h}-\mathrm{ph}}\left[\alpha_{\mathrm{tJH}}(\omega)\right] .
$$

Weak and anisotropic interaction with magnons is treated in NCA:

$$
\Sigma_{\mathrm{h}-\mathrm{mag}}^{\mathrm{SCBA}}(\mathbf{k}, \omega)=\sum_{\mathbf{q}} \frac{M_{\mathbf{k}, \mathbf{q}}^{2}}{\omega-\omega_{\mathbf{q}}-\Sigma_{\mathrm{t} J \mathrm{H}}\left(\mathbf{k}-\mathbf{q}, \omega-\omega_{\mathbf{q}}\right)+i \varepsilon},
$$

while the function $\alpha(\omega)$ for the phonon part of the hole selfenergy

$$
\alpha_{\mathrm{tJH}}(\omega)=\frac{1}{N} \sum_{\mathbf{k}} \frac{1}{\omega-\Sigma_{\mathrm{h}-\mathrm{mag}}^{\mathrm{SCBA}}(\mathbf{k}, \omega)+i \varepsilon}
$$

is expressed in terms of momentum-averaged bare Green function whose momentum dependence is determined by magnetic self-energy (16) obtained in NCA. Usage of MA instead of DMFT in (16) is crucial since the DMFT even does not distinguish even between $\mathrm{t}-\mathrm{J}_{z}$ and $\mathrm{t}-\mathrm{J}$ model [223]. To the contrary, results obtained from (13)-(16), as it is shown by comparison with DMC data [42], correctly reproduce not only the ground state properties but the spectral function too.

The advantage of the suggested in [50] approach is that it is easily generalized to finite temperatures. Transforming magnetic self-energy $\Sigma_{\mathrm{h} \text {-mag }}^{\mathrm{SCBA}}(\mathbf{k}, \omega)[208,234]$ to the Matsubara form, one gets

$$
\begin{aligned}
\Sigma_{\mathrm{h}-\mathrm{mag}}^{\mathrm{SCBA}}(\mathbf{k} \omega)= & \sum_{\mathbf{q}} \frac{M_{\mathbf{k}, \mathbf{q}}^{2}\left(1+n_{b}\left(\omega_{\mathbf{q}}\right)\right)}{\omega-\omega_{\mathbf{q}}-\Sigma_{\mathrm{t} \mathrm{H}}\left(\mathbf{k}-\mathbf{q}, \omega-\omega_{\mathbf{q}}\right)+i \varepsilon} \\
& +\sum_{\mathbf{q}} \frac{M_{\mathbf{k}+\mathbf{q}, \mathbf{q}}^{2}\left(n_{b}\left(\omega_{\mathbf{q}}\right)\right)}{\omega+\omega_{\mathbf{q}}-\Sigma_{\mathrm{t} \mathrm{H}}\left(\mathbf{k}+\mathbf{q}, \omega+\omega_{\mathbf{q}}\right)+i \varepsilon},
\end{aligned}
$$

where $n_{b}(\omega)$ is the Bose distribution. To generalize the phonon self-energy (13) to finite temperatures one uses the momentum-independent relation $[225,226]$

$$
\Sigma_{\mathrm{h}-\mathrm{ph}}[\alpha(\omega)]=\alpha^{-1}(\omega)-\sum_{n=0}^{\infty} \frac{(1-x) x^{n}}{\alpha^{-1}(\omega)-A_{n}(\omega)-B_{n}(\omega)}
$$

Here $x=\exp \left(-\beta \Omega_{0}\right)$, and $A_{n}(\omega)$ and $B_{n}(\omega)$ are known functions depending on the coupling constant and frequency [50]. Also, (14), (16), (17), and (18) are solved selfconsistently. The spectral functions obtained from these equations obey three first sum rules for any coupling constants and temperatures $[212,220]$. Hence, the peak position and its width should be reliable.

Temperature dependence of the spectral function in the ground state $k=(\pi / 2, \pi / 2)$ for different $\lambda \mathrm{s}$ is shown in Figure 8 . The general trends are in agreement with experiments $[60,109,177,235]$. Distance of the Franck-Condon peak from the chemical potential and its width increases with the increase of temperature. Note that peak width is a constant up to the temperature $T \simeq \Omega_{0} / 2 \approx 200 \mathrm{~K}$ and then show a linear temperature dependence (see Figure 8). This linear dependence, if no data for low temperatures, can be indeed extrapolated to wrong low temperature values. However, in accordance with recent experiments $[60,236]$, the linewidth saturates at temperatures $T \leq \Omega_{0} / 2$ to a constant value. Note that the slope of the temperature dependence for $T>\Omega_{0} / 2$ does not depend on $\lambda$ whereas the saturation values of the linewidth for $T<\Omega_{0} / 2$ are very sensitive to the strength of EPI.

Hence, temperature dependence of the Franck-Condon peaks in the low-energy $(0.3-0.6 \mathrm{eV})$ part of the ARPES spectra manifests strong EPI. The evidence of the strong EPI was recently found in the high-energy ARPES too. It follows from the temperature dependence of high-energy "waterfall" features in ARPES [63]. The "waterfalls" at the energies $1-2 \mathrm{eV}$ are the parts of the ARPES spectra where dispersion is parallel to the energy axis in the energymomentum reference frame [178-182]. At first, the structure of "waterfalls" was reproduced by artificial broadening of the spectra of the $t-J$ model $[183,184]$. Then, an attempt to explain the artificial broadening by finite temperature and EPI was made in [63]. The method of self-consistent treatment of Dyson equation $[68,69,237]$ was generalized to finite temperature and EPI is added. It is concluded [63] that the finite temperature alone is not enough to explain the effect and strong EPI is compulsory to explain the data.

Another explanation of the "waterfalls" was suggested in [238] where spectral density at large energies is attributed to contribution of localized states.

3.7. Multipeak Structure of the Optical Conductivity Mediated by EPI. The optical conductivity (OC) of the weakly doped cuprates has the following characteristic features $[23,24,70]$. The high-energy part of OC in undoped systems has the peak at $\omega \cong 1.5 \mathrm{eV}$ which corresponds to charge transition between p-orbitals of $\mathrm{O}$ and d-orbitals of $\mathrm{Cu}$. Low-energy part of OC, caused by movement of holes, arises with doping. The Drude contribution at the lowest energy in proportional to the holes concentration $x$ and the relaxation rate of the Drude theory is proportional to temperature. Puzzling part of the OC is the mid infrared (MIR) peak with doping-dependent frequency around $\omega_{\mathrm{MIR}} \cong 0.5 \mathrm{eV}$ whose interpretation is still far from adopted [25]. Theoretically the OC of $\mathrm{t}-\mathrm{J}$ model was studied in the low density limit and a peak at $2 J \approx 0.28 \mathrm{eV}$ was found [9-22] (for typical $t=0.4 \mathrm{eV}$ 

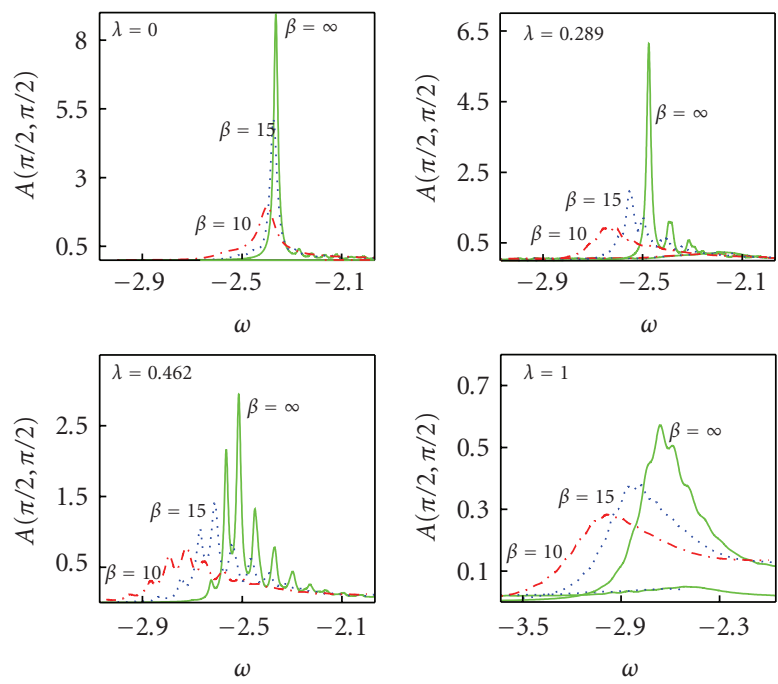

(a)

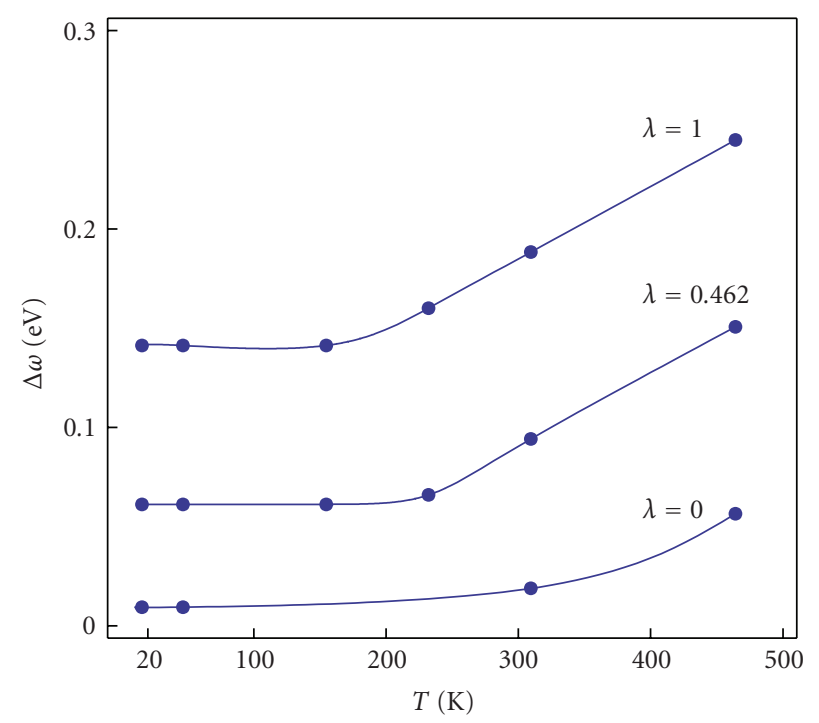

(b)

Figure 8: (a) Spectral functions for different $\lambda$ and temperatures $\beta=t / T$. (b) Peak width $\Delta \omega$ as a function of temperature $T$ for the t-J model $(\lambda=0)$ and t-J-H model in the strong coupling EPI limit $(\lambda=0.462$ and $\lambda=1)$. Temperature is in Kelvins under assumption that $t=0.4 \mathrm{eV}$.

and $J=0.35 t$ ). The above energy is almost two times smaller than the low-doping experimental value $0.5 \mathrm{eV}$. Hence, to shift theoretical peak of the $t-J$ model to higher energies, one can try to add EPI and consider $\mathrm{t}-\mathrm{J}-\mathrm{H}$ model.

However, OC of the t-J-H model was studied by ED [165], using NCA both for phonons and magnons [167], and by DMFT method which is exact in the infinite dimension limit [224]. NCA is not valid for strong EPI and small systems in ED method give too discrete spectrum. Beside, it is not known whether infinite dimension is a good approximation for the two-dimensional (2D) system. To circumvent the above difficulties, the OC was calculated in [51] by DMC method for 2D infinite system and without NCA in the phonon channel. Theoretical data were compared with experimental ones obtained by ellipsometry, Figure 9 shows OC (a) in Holstein model, (b) t-J model and (c) t-J-H model. Experimental result is in Figure 9(d). No model, except the $\mathrm{t}-\mathrm{J}-\mathrm{H}$ one, reproduces even the gross features of the experiment. The most striking feature is the two-peak structure of MIR band. There is an MIR peak at $\omega_{\text {MIR }}=$ $4600 \mathrm{~cm}^{-1}$ and a low-energy peak at $\omega=1000 \mathrm{~cm}^{-1}$ which is situated just above the phonon lines at around $800 \mathrm{~cm}^{-1}$. Note that in the t-J model, the MIR peak is at $\omega_{\mathrm{t}-\mathrm{J}} \cong 2 J \cong$ $2000 \mathrm{~cm}^{-1}$. However, EPI of $\mathrm{t}-\mathrm{J}-\mathrm{H}$ model shifts the peak to its experimentally observed frequencies.

It is shown in [51] that the lower peak of OC originates from the phonon-mediated scattering of the hole between the states located in the coherent $\mathrm{t}-\mathrm{J}$ band and the threshold of the optical absorption corresponds to the phonon energy. The higher energy peak is a magnetic satellite of the lower peak. Note that its energy is higher in comparison with system where EPI is set to zero. Assuming strong coupling regime, when one can take advantage of the Franck-Condon scheme [48], one can explain this shift to higher energies as follows. The fluctuations of the energies on different sites of the lattice are around the Franck-Condon relaxation energy. On the other hand, these fluctuations are frozen during the characteristic time of optical transition. Hence, the energy of transition of the hole from the ground to excited state is the sum of the Franck-Condon energy and that of excited magnon.

Figure 10 shows how OC changes with increase of $\lambda$. OC at weak coupling shows low-energy peak which intensity starts just above the phonon energy and there is apparent two-peak structure of the absorption. Actually, the twopeak structure was observed in experiment even in early measurements $[23,27,115-120]$. Especially apparent twopeak structure is seen in OC measured by ellipsometry in the weakly doped $\mathrm{Eu}_{1-x} \mathrm{Ca}_{x} \mathrm{Ba}_{2} \mathrm{Cu}_{3} \mathrm{O}_{6}$ (see Figure 9(d)). The low-energy peak is close to the phonon energy up to $\lambda \approx 0.4$ when the system comes into the strong coupling regime [42] and for $\lambda>0.4$ all peaks quickly enhance their energies (see Figure 11(a)). To reveal the nature of the low-energy peak the OC of the effective Holstein model was studied. In this model, the hopping integral was chosen $\widetilde{t}=0.4 t$ to reproduce the enhancement of the mass in the t-J model. Besides, the crossover to the strong coupling regime occurs in the effective model at $\lambda \approx 0.4$. The OC of such model well reproduces the low-energy part of the t-J-H model (see Figure 11(b)).

Doping dependence of the kink angles in ARPES [64] suggests that the EPI strength decreases with doping and, hence, experimentally observed softening of the MIR energy with doping $[25,26,28]$ is because of decrease of EPI. Theoretically, the decrease of the effective EPI with doping was found for a gas of Fröhlich polarons [239-242]. Comparison of the experimental position of the MIR peak with the results of the $\mathrm{t}-\mathrm{J}-\mathrm{H}$ model gives the estimate [51] for change of the 


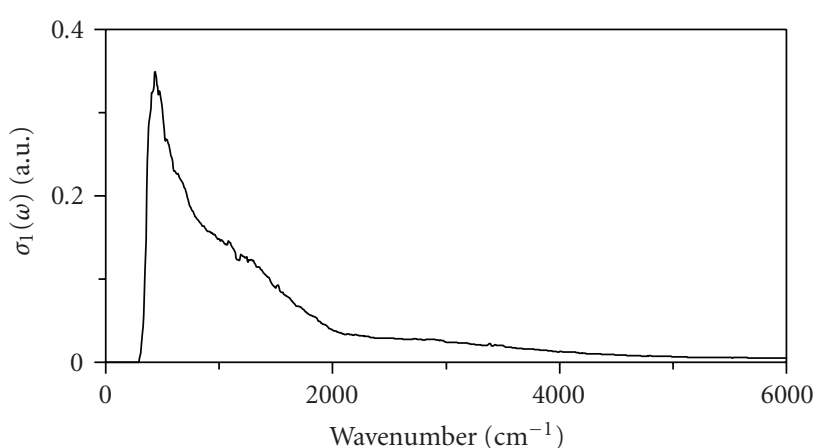

(a)

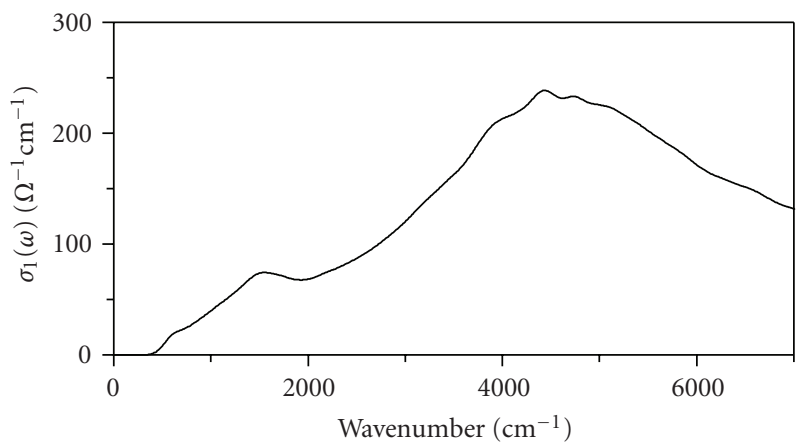

(c)

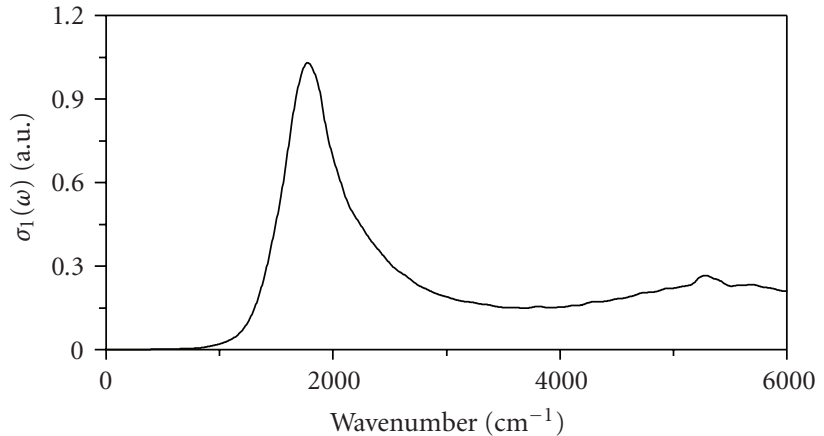

(b)

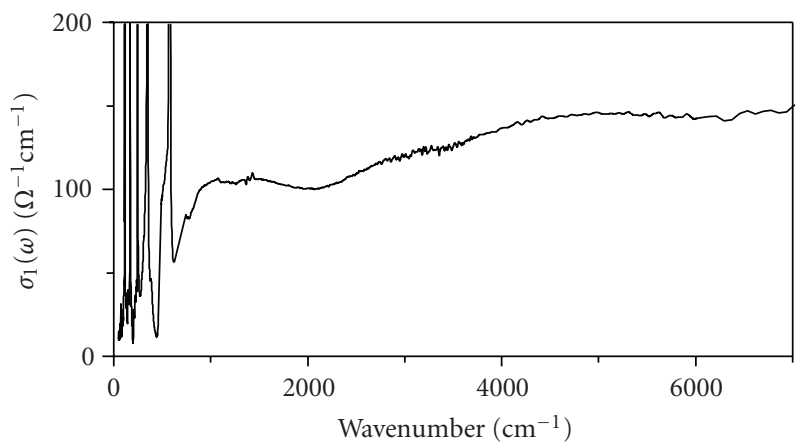

(d)

FIGURE 9: Typical OC of different models in two-dimensions and experimental data in weakly doped system: (a) Holstein model at $\lambda=0.44$; (b) t-J model at $J=0.3$; (c) t-J-H at $J=0.3$ and $\lambda=0.39$; (d) in-plane OC of doped by $x=1.5 \%$ holes $\mathrm{Eu}_{1-x} \mathrm{Ca}_{x} \mathrm{Ba}_{2} \mathrm{Cu}_{3} \mathrm{O}_{6}$ at $T=10 \mathrm{~K}$. Theoretical energy scale assumes $t=0.3 \mathrm{eV}\left(1 \mathrm{eV}=8065.5 \mathrm{~cm}^{-1}\right)$. The absolute values of the theoretical $\sigma_{1}$ estimated assuming that hopping distance in plane is $a=3.86 \AA$ and the bulk concentration of holes is $n_{h}=1.72 \times 10^{-23} \mathrm{~cm}^{-3}$.

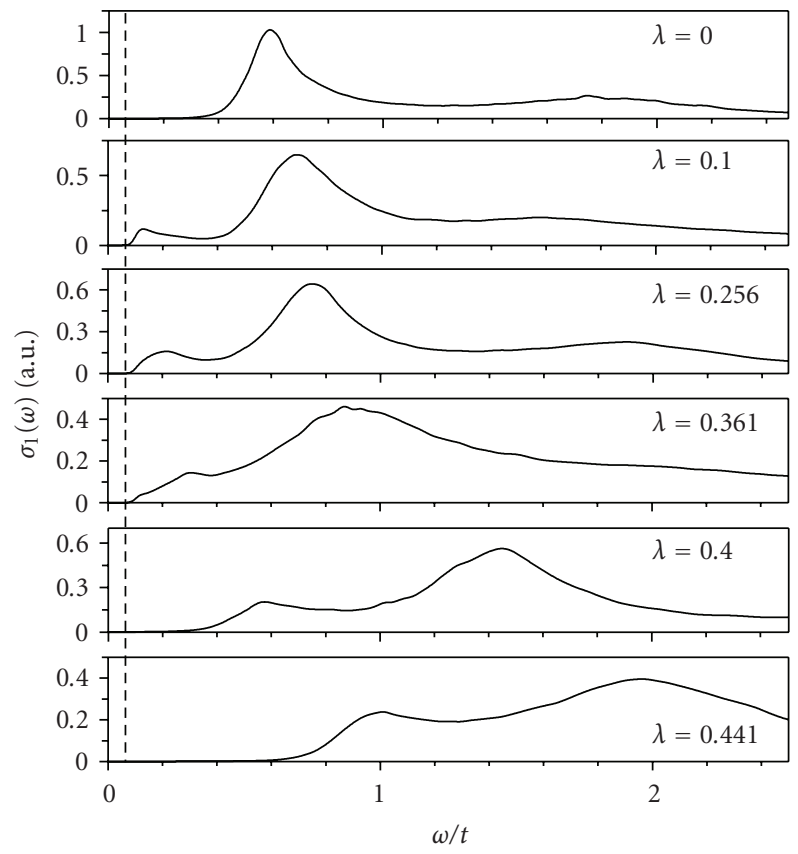

Figure 10: Optical conductivity of a one hole in the t-J-H model calculated by DMC method at $J / t=0.3$ for different $\lambda$. Vertical line at $\omega / t=0.1$ shows phonon energy. effective EPI with doping (Figures 11(c) and 11(d)). The figures show the ratio of the coupling constant at the given doping to that at zero doping. However, since zero doping is characterized by the coupling constant $\lambda(x=0) \approx 1$ (see Section 3.4), one can think of the data in Figures 11(c) and $11(\mathrm{~d})$ as showing the absolute value of $\lambda(x)$. Note very similar behavior of $\lambda(x)$ for different compounds. It is clear that the compounds become superconducting when the system is not already in the strong coupling regime of the EPI. However, the value of the EPI strength $\lambda \approx 0.5$ is still considerable.

Alternative explanation of the doping dependence of the MIR energy by the doping dependence of the exchange integral $J(x)$ was suggested in $[224,243]$. One cannot distinguish between $J(x)$ - and $\lambda(x)$-scenario just looking on OC. However, $\lambda(x)$-scenario easily explains experimentally observed strong dependence of the kink angle on concentration $[64,97,121,128]$. To the contrary, $J(x)$-approach $[224,243]$ does not give an explanation. Experimental data in electron doped $\mathrm{Nd}_{2-x} \mathrm{Ce}_{x} \mathrm{CuO}_{4}$ were interpreted as indicating that $\lambda$ does not show doping dependence [145]. However, the range where $\lambda$ is determined in [145] is too narrow to reach the final conclusion.

The polaronic scenario was used to interpret OC in cuprates many times [115, 242, 245-249]. However, all these cited papers discussed the one-peak structure and did not describe the complex two-peak structure of the 


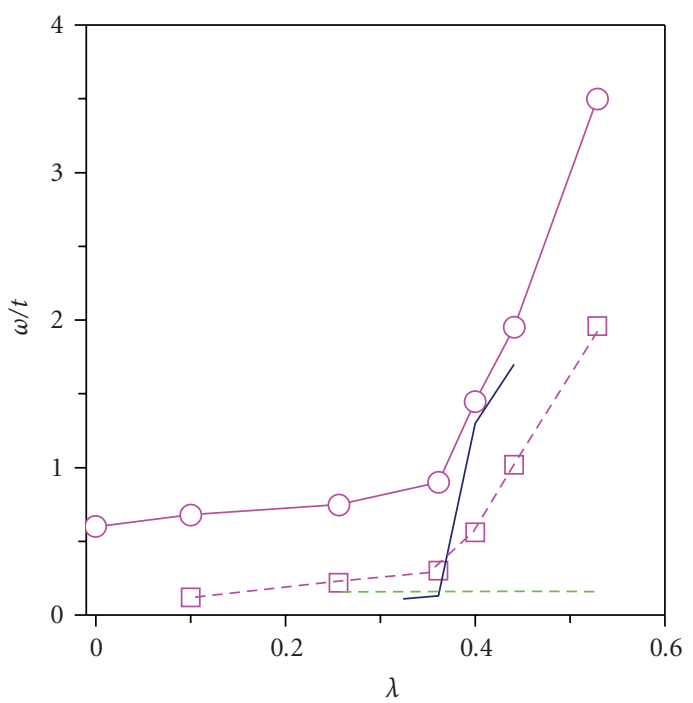

(a)

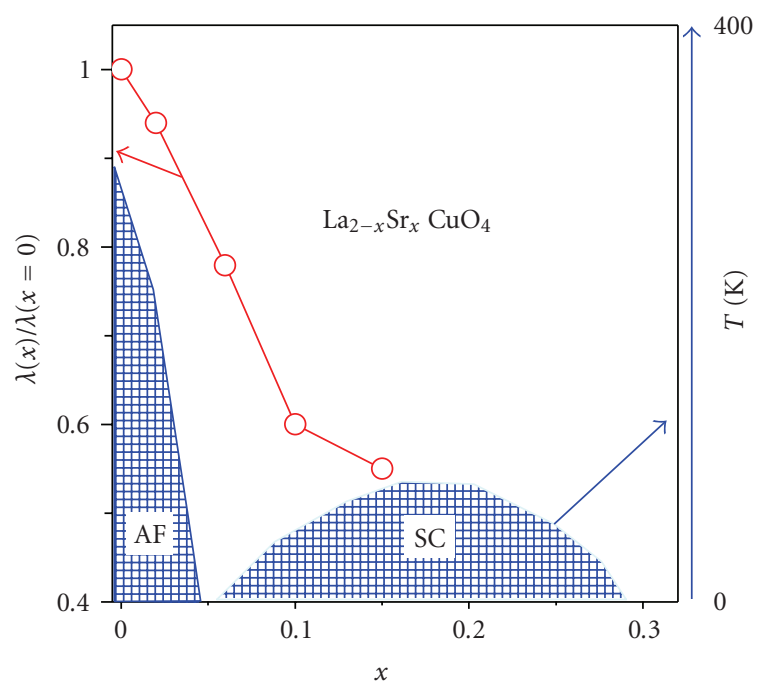

(c)

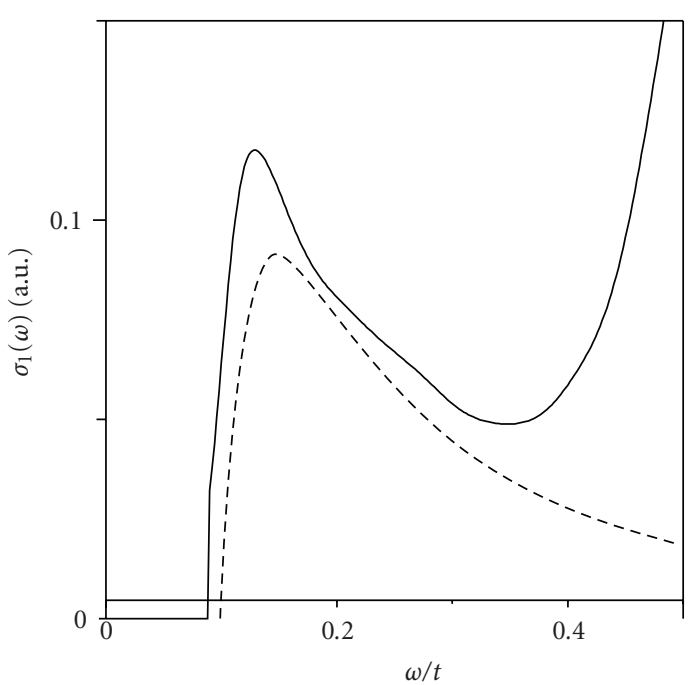

(b)

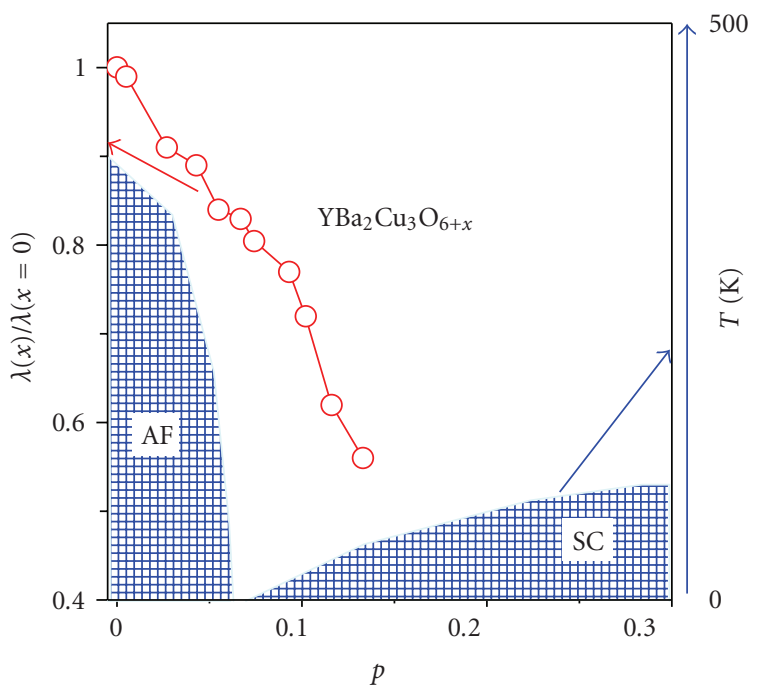

(d)

FIGURE 11: (a) Energies of the dominant MIR peak (solid line with circles) and low-energy peak (dashed line with squares) of the t-J-H model versus $\lambda$. Energies of the dominant peaks of the Holstein model for $t=1$ (dashed line) and for $\tilde{t}=0.4$ (solid line). (b) OC of the $\mathrm{t}-\mathrm{J}-\mathrm{H}$ model (solid line) and effective Holstein model with $\tilde{t}=0.4$ (dashed line). Both OC are for $\lambda=0.1$. (c) and (d) Ratio of the effective coupling constants on doping $x$ (or real concentration $p$ of the hole in plain for YBCO [244]) and that at almost zero doping. Effective $\lambda(x)$ is determined by the fit of the MIR position at given doping.

experimental spectra. One of exceptions where two-peak structure is discussed is [250]. It is pointed out that the two-peak structure of OC occurs at some coupling constants in the Fröhlich model $[41,48]$. There is also some range of coupling constants where two-peak structure of OC is seen in the Holstein model [251]. However, one needs fine tuning of the EPI strength to get the two-peak OC in the Fröhlich or Holstein model. To the contrary, the two-peak structure is the robust property of the experimental data and the OC of the t-J-H model. The authors of [250] used similar approaches $[252,253]$ to calculate the OC and ARPES spectra and found clear relation between the position of the peaks in ARPES and OC spectra.

Another interpretation of the low-energy peak of OC is given in [254], where the peak is considered to be of the purely magnetic origin. It seems that in the framework of the interpretation given in [254] the low-energy peak is connected with local magnetic excitations. Therefore, the nature of the low-energy peak is still under debate theoretically although experimentally it is a vivid feature of the OC of many cuprates with different doping levels $[23,27,115-120]$. 
3.8. Nonlocal EPI. The simplest model (3)-(7) does not reproduce all peculiarities of cuprates. The reason is that actually EPI vertex depends on momenta of both hole and phonon $[61,82,85-90,92,93]$. As a result, some properties of the t-J-H model do not match experimental data. For example, effective mass of a hole in the strong coupling regime is very large which is in contradiction with rather moderate masses of carriers observed in experiment [25]. Even more profound disagreement with experiment was found in [210]. It was shown there that in the strong coupling regime of the $\mathrm{t}-\mathrm{J}-\mathrm{H}$ model the mobile hole is transformed into the localized one with four broken bonds around it. In such case, the percolation model predicts that the antiferromagnetic model survives up to hole concentration $x=0.5$ which is in severe disagreement with experiment where antiferromagnetic phase is limited by the doping concentrations $x \leq 0.02-0.04$. Similar trend was noted in [255] where it was demonstrated that EPI helps to survival of the antiferromagnetism since EPI suppresses the motion of the holes which is necessary to suppress antiferromagnetism.

The minimal Hamiltonian of the $\mathrm{t}-\mathrm{t}^{\prime}-\mathrm{t}^{\prime \prime}-\mathrm{J}$ model with nonlocal EPI consists of the sum of the Hamiltonian of the $t$ $\mathrm{t}^{\prime}-\mathrm{t}^{\prime \prime}$-J model, phonon Hamiltonian with phonon frequency $\omega_{0}$, and the Hamiltonian of nonlocal EPI

$$
\begin{aligned}
H_{\mathrm{h}-\mathrm{ph}}= & \omega_{0} \sum_{l} g(l) \sum_{i \in A} f_{i}^{\dagger} f_{i}\left(c_{i+l}^{\dagger}+c_{i+l}\right) \\
& +\omega_{0} \sum_{l} g(l) \sum_{i \in B} h_{i}^{\dagger} h_{i}\left(c_{i+l}^{\dagger}+c_{i+l}\right),
\end{aligned}
$$

which is defined in terms of local coupling constant $g(0)=$ $g$ and nonlocal coupling to the displacements of near neighbors $g(\vec{\delta})=g_{1}$. However, $f$ and $h$ denote annihilation operators of two sublattices of the two-dimensional antiferromagnetic lattice.

The problem of nonlocal EPI in $t-t^{\prime}-t^{\prime \prime}-J$ model was solved in [49] with a novel approach. In that approach, starting from the state of a hole in an antiferromagnet, one defines the states of the basis $|h\rangle_{j}\left[\prod_{i}\left|\mu_{i}\right\rangle\right]\left|\vec{q}_{1}, \ldots, \vec{q}_{l}, l\right\rangle$. Here $|h\rangle_{j}$ is a hole at site $j$ and $i$ runs through the whole lattice. The set of states $\left|\vec{q}_{1}, \ldots, \vec{q}_{l}, l\right\rangle$ is limited by magnons which component of the lth order is enough to reproduce the results of NCA [256]. As it is shown in [49], $l=4$ is enough for $J / t \geq 0.3$ since diagonalization of this $l \leq 4$ basis in the retraceable path approximation [257] gives results reproducing those of the NCA.

However, the hardest problem for the numeric solution comes from the phonon basis. Exponential growth of the phonon basis with coupling previously limited the system sizes to 10 sites [165]. The problem is circumvented by usage of the coherent states (CS) $[258,259]$ which are the canonical transformations of the phonon basis:

$$
|h, i\rangle=e^{g h\left(b_{i}-b_{i}^{\dagger}\right)}|0\rangle_{i}^{(\rho h)}=e^{-g^{2} h^{2} / 2} \sum_{n=0}^{\infty} \frac{(-g h)^{n}}{\sqrt{n !}}|n\rangle_{i}
$$

with free parameter $h$. Such approach can treat $8 \times 8$ lattices which are the largest systems ever treated for $\mathrm{t}-\mathrm{J}$ and related models which include coupling to phonons. CS with $h=0$ is the bare state and for $h=1$ it is the exact solution to the independent oscillators model with local EPI coupling g. A comparison with DMC data showed [49] that four CSs are enough for reliable treatment of $t-t^{\prime}-t^{\prime \prime}-J$ model in all coupling regimes.

The $\mathrm{tt}^{\prime} \mathrm{t}^{\prime \prime} \mathrm{J}-\mathrm{H}$ model was compared in [49] with the model where interaction with the displacements on near neighbors $g_{1}=g / 2$ (see (19)) was added. Figure 12 shows dependence on $\lambda / \lambda_{c}$ of the ground state spectral weight $Z$, spin deviation $\mathrm{SD}$, and magnon-mediated kinetic energy $K_{t}$. Spin deviation $\mathrm{SD}=\left(S_{\mathrm{AFM}}-\left\langle S_{\mathrm{NN}}\right\rangle\right) / S_{\mathrm{AFM}}$ is a measure of how the spin $S_{\mathrm{NN}}$ on the neighboring to the hole site deviates from the value of spin $S_{\text {AFM }}$ in the ideal antiferromagnet. The decrease of $Z$ is a measure of suppression of coherent motion of the hole whereas the absolute values of $K_{t}$ and SD are the measures of intensity of the near neighbor hoppings. Decrease of $K_{t}$ and SD indicates suppression by EPI of the movement of a hole on near neighbors.

Nonlocality of the EPI is manifested in the following features. Coherent motion is suppressed stronger at $\lambda<\lambda_{c}$ and weaker at $\lambda>\lambda_{c}$ (see Figure 12(a)). Also, intensity of the near neighbors hoppings is considerably weaker suppressed by nonlocal EPI than by the local one. The last statement is easy to illustrate in the strong coupling regime where one can think in terms of the adiabatic potential. The adiabatic potential in the strong coupling regime is a $\delta$-function giving no possibility of a motion of the hole over the near neighbors. To the contrary, for long-range EPI, the adiabatic potential is not steep and, thus, the motion of the hole over the near neighbors is not suppressed even in the strong coupling limit. Such mobility over the near neighbor gives possibility to destroy the antiferromagnetic state even in the strong coupling regime. Besides, the polaron with nonlocal EPI is lighter in the strong coupling regime. For example, for $\lambda=1.1 \lambda_{c}$ the diagonal $m_{d}\left(k_{x}=k_{y}\right)$ and transverse $m_{t}$ $\left(k_{x}=-k_{y}\right)$ masses for the local EPI are $m_{d}=200$ and $m_{t}=88$ whereas for nonlocal EPI the masses $m_{d}=20$ and $m_{t}=10$ are considerably lighter. Lighter mass for nonlocal EPI $[98,195,260-265]$ or dispersive phonons [266-269] was observed in many models.

Common influence of long-range EPI and on site repulsion binds polarons into mobile inter-site bipolarons $[270,271]$. For peculiar lattice structures such polarons can be very light [272].

Manifestation of the nonlocality of the EPI can be seen in the OC $[4,273]$. For example, the peak in the OC of the Holstein model in the strong coupling regime is seen on the energy $2 \epsilon_{p}$, where $\epsilon_{p}$ is the polaron binding energy. On the other hand, the peak of OC in the models with long-range EPI is on the considerably smaller energies [273]. It can be explained by the fact that OC is defined in terms of the current-current correlation function. The current operator in the polaronic models with near neighbor hopping corresponds to the transition of a quasiparticle to the neighboring site. The shifted electron in the Holstein model looses the energy $\epsilon_{p}$ and leaves the excited phonons with the same energy on the initial site. For the long-range EPI the phonons are excited on the polaron site and on the 


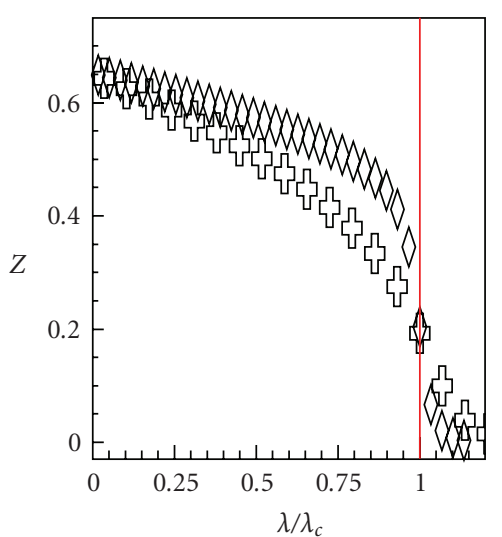

(a)

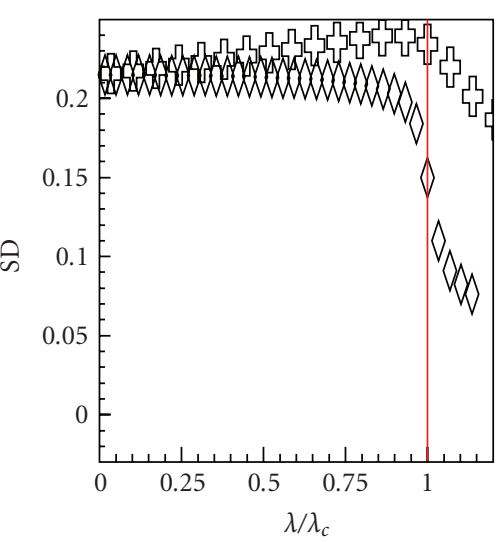

(b)

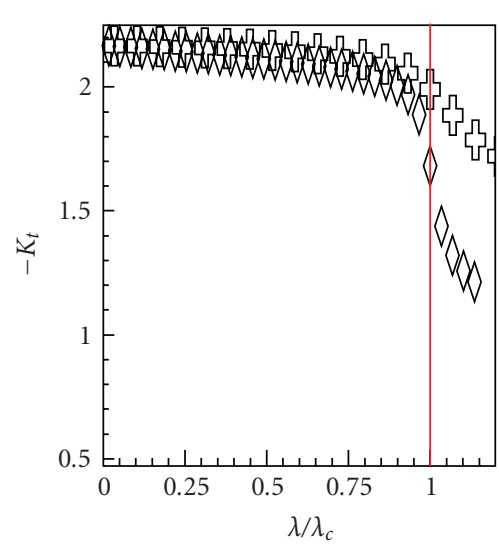

(c)

FIGURE 12: (a) Spectral weight, (b) spin deviation (b), and (c) average kinetic energy of mediated by magnons near neighbor transitions for $g_{1}=0$ (diamonds) and $g_{1}=0.5 g$ (crosses). Other parameters are $J / t=0.4, \omega_{0} / t=0.2, t^{\prime}=-0.5 t, t^{\prime \prime}=0.4 t$, and $N=4 \times 4=16$. Critical $\lambda_{c}$ is set at such coupling where the spectral weight of the ground state reaches 0.3 of the unrenormalized by EPI weight.

neighboring sites which leads to considerably weaker lattice relaxation during transition to the neighboring site. Hence, the energy of the peak in OC for nonlocal EPI is smaller.

The peculiarities of the influence of nonlocal EPI is determined not only by the range but also by its fine structure. Comparison of buckling and breathing phonons is made in [274]. In the Hamiltonian:

$$
H_{\mathrm{Br}-\mathrm{Bu}}=\gamma \sum_{\mathbf{i}, \delta}\left(b_{\mathbf{i}, \delta}+b_{\mathbf{i}, \delta}^{\dagger}\right)\left(n_{\mathbf{i}} \pm n_{\mathbf{i}+\boldsymbol{\delta}}\right),
$$

where $\mathbf{i}$ is position of cooper and $\boldsymbol{\delta}=x, y$ is the link direction. Plus (minus) corresponds to buckling (breathing) mode. It is shown [274] that the breathing mode suppresses the kinetic energy considerably stronger than it is done by buckling mode. Breathing phonon decreases the energy of one site and increases it on the neighboring one. To the contrary, the buckling mode changes the energy of the neighboring sites in the same direction and, hence, does not suppress the kinetic energy effectively. Studies of influence of the EPI structure on the properties of polarons can be found in $[98,194,195,262,275]$.

\section{Electronic Correlations and EPI}

Manifestations of the EPI in the properties of particles and phonons are profoundly different although the first naive but correct impressions is that both elementary excitations become softer and broader. Let a hole (phonon) in a system without EPI has dispersion $\varepsilon(\mathbf{k})=\epsilon(\mathbf{k})(\varepsilon(\mathbf{k})=$ $\omega(\mathbf{k}))$. In such system, experimentally observed response in ARPES (neutron scattering) is expressed in terms of spectral function $S(\mathbf{k}, \omega)$ having the form of a delta function $S(\mathbf{k}, \omega)=\delta(\omega-\varepsilon(\mathbf{k}))$. EPI leads to additional self-energy part of the Green function of a hole (phonon) $\Theta(\mathbf{k}, \omega)=\Sigma(\mathbf{k}, \omega)$
$(\Theta(\mathbf{k}, \omega)=\Pi(\mathbf{k}, \omega))$. With this self-energy, the spectral function, measured in experiment, reads

$$
S(\mathbf{k}, \omega)=\frac{1}{\pi} \frac{|\operatorname{Im} \Theta(\mathbf{k}, \omega)|}{[\omega-\varepsilon(\mathbf{k})-\operatorname{Re} \Theta(\mathbf{k}, \omega)]^{2}+[|\operatorname{Im} \Theta(\mathbf{k}, \omega)|]^{2}} .
$$

Imaginary part $|\operatorname{Im} \Theta(\mathbf{k}, \omega)|$ determines the EPI-driven broadening.

There is a sum rule for phonon self-energy in the $t$ J model (in the $U \rightarrow \infty$ limit of Hubbard model) when charge fluctuations in the half-filled system are completely suppressed $[83,276]$ :

$$
\frac{1}{\pi N} \sum_{\mathbf{k}} \int_{-\infty}^{\infty}|\operatorname{Im} \Pi(\mathbf{k}, \omega)| d \omega \approx \gamma^{2}[2 \delta(1-\delta)] .
$$

Here $N$ is number of sites and $\delta$ is concentration of holes which is zero in half-filled system. Naturally, with no empty spaces in half-filled system there is no charge fluctuations at all and the phonons are left untouched. Increase of $\delta$ adds empty states and the charge fluctuations renormalize and broaden phonons. To the contrary, one does not need doping to see the manifestations of EPI in ARPES spectra. For $\delta=0$ the sum rule for the hole self-energy reads [276]

$$
\frac{1}{\pi} \int_{-\infty}^{0} \operatorname{Im} \Sigma\left(\mathbf{k}, \omega-i 0^{+}\right) d \omega=\gamma^{2} .
$$

Thus, half-filled system does not suppresses EPI in the ARPES channel. The reason is that ARPES creates its own hole even in the half filled system. This hole is a charge whose fluctuations are not suppressed (see also [277]).

In general, $U$ suppresses EPI. Also, DMFT calculations show [278-280] that EPI in paramagnetic system is severely depressed by correlations. Antiferromagnetic state considerably enhances the role of EPI in comparison with paramagnetic system [281]. Doping suppresses the EPI contribution to the electronic properties. To the contrary, 
contribution of EPI into the phonon properties increases with doping (cf. (23)).

In any case, for small filling of the t-J model, one can conclude that in comparison with the Holstein model with small filling the influence of the EPI is larger for the $t-J$ model. This result was verified by a number of calculations $[42,165,282-284]$. Comparing critical coupling of the t-J$\mathrm{H}$ (3)-(7) model $\lambda_{\mathrm{t}-\mathrm{J}}^{c} \approx 0.4$ [42] with the critical coupling of the Holstein model with the same hopping $t \lambda_{H}^{c} \approx 1$, one concludes that the interaction of the hole with magnons makes the transition into the strong coupling regime faster.

For a hole in the bottom of the band the ratio of effective $\lambda$ for the t-J model and $\lambda_{0}$ for the Holstein model with bare mass $m_{0}=1 /(2 t)$ depends on numerous factors $[4,166$, 171]:

$$
\frac{\lambda}{\lambda_{0}} \approx 4 Z_{0}^{2} \frac{\sqrt{m_{\|} m_{\perp}}}{m_{0}} .
$$

The decreasing factor $Z_{0}<1$ arises because of the shift of the spectral density to higher frequencies. On the other hand, larger effective masses of the t-J model $m_{\|}>m_{0}$ and $m_{\perp}>m_{0}$ enhance the influence of EPI. The factor 4 arising because of 4 -fold degeneracy of the ground state on the wave vectors $( \pm \pi / 2, \pm \pi / 2)$ becomes sometimes decisive. At $J / t=0.2$, one has $Z_{0}^{2}=0.05$ and $\sqrt{m_{\|} m_{\perp}}=10 m_{0}$. In this case there is doubling of the EPI, partly due to the factor 4 . For large $J / t=2$, the enhancement of EPI $\lambda / \lambda_{0} \approx 16$ can be very large. One can conclude that the change of the role of EPI usually has no universal trends and very often determined by fine features of the model, dimensionality, filling, and so forth. For example, the role of EPI is suppressed by Coulomb repulsion in the ground state of one-dimensional Mott insulator [285] whereas its role in formation of the exciton spectrum is enhanced by the same Coulomb repulsion [286].

One of the factors influencing the role off EPI in cuprates is typical for cuprates inhomogeneity [287-290]. Moderate and even weak EPI can lead to dramatic changes of the properties of inhomogeneous electronic gas [291]. Interfaces and surfaces are another inhomogeneities enhancing the role of EPI [292]. Similar conclusions can be drawn from many numeric calculations [53, 293-298].

Current review is restricted to theoretical approaches considering strong correlations. The significant number of important results of band structure calculations, the incomplete list is [299-310], revealing large $\lambda \geq 1$ value of EPI, are not discussed here because of space limitation.

\section{Conclusions}

This review presents a lot of evidences for the important role of the EPI in formation of the spectral properties of underdoped cuprates. Theoretical efforts to reveal the fingerprints of the EPI in spectral response went through the sequence of models with increasing complexity. The simplest models with short range EPI in the ideal lattice at zero temperature were the systems to start with. Then, the progress went through generalizations to finite temperatures, to nonlocal EPI, and to systems with imperfections.
The ultimate goal of theory is to describe the realistic situation in cuprates. Such goal requites further development of the numeric approaches since the realistic description of cuprates requires methods which are capable of describing an infinite systems with imperfections at finite temperature.

\section{Acknowledgments}

This review is supported by RFBR 07-02-0067a. The author acknowledges stimulating discussions with N. Nagaosa, N. V. Prokof'ev, B. V. Svistunov, Yu. Kagan, A. S. Alexandrov, G. De Filippis, V. Cataudella, A. Sakamoto, Z.-X. Shen, T. P. Devereaux, C. Bernhard, J. Zaanen, G. Sawatzky, K. M. Shen, J. Bonča, E. Cappelluti, S. Ciuchi, M. Berciu, T. Egami, J. T. Devreese, T. Tohyama, O. Gunnarsson, G. Sangiovanni, and C. Kim.

\section{References}

[1] P. W. Anderson, The Theory of Superconductivity in the High$T_{c}$ Cuprates, Princeton University Press, Princeton, NJ, USA, 1997.

[2] A. S. Alexandrov, "Bose-Einstein condensation of strongly correlated electrons and phonons in cuprate superconductors," Journal of Physics: Condensed Matter, vol. 19, no. 12, Article ID 125216, 2007.

[3] A. S. Alexandrov, "Bipolaren anisotropic flat bands, Hall mobility edge, and metal-semiconductor duality of overdoped high- $T_{c}$ oxides," Physical Review B, vol. 53, no. 5, pp. 2863-2869, 1996.

[4] O. Gunnarsson and O. Rösch, "Interplay between electronphonon and Coulomb interactions in cuprates," Journal of Physics: Condensed Matter, vol. 20, no. 4, Article ID 043201, 2008.

[5] A. Damascelli, Z. Hussain, and Z.-X. Shen, "Angle-resolved photoemission studies of the cuprate superconductors," Reviews of Modern Physics, vol. 75, no. 2, pp. 473-541, 2003.

[6] T. Xiang and J. M. Wheatley, "Quasiparticle energy dispersion in doped two-dimensional quantum antiferromagnets," Physical Review B, vol. 54, no. 18, pp. R12653-R12656, 1996.

[7] V. I. Belinicher, A. L. Chernyshev, and V. A. Shubin, "Generalized $t-t^{\prime}-\mathrm{J}$ model: parameters and single-particle spectrum for electrons and holes in copper oxides," Physical Review B, vol. 53, no. 1, pp. 335-342, 1996.

[8] V. I. Belinicher, A. L. Chernyshev, and V. A. Shubin, "Singlehole dispersion relation for the real $\mathrm{CuO}_{2}$ plane," Physical Review B, vol. 54, no. 21, pp. 14914-14917, 1996.

[9] T. M. Rice and F. C. Zhang, "Frequency-dependent conductivity from carriers in Mott insulators," Physical Review B, vol. 39, no. 1, pp. 815-818, 1989.

[10] W. Stephan and P. Horsch, "Optical properties of one- and two-dimensional Hubbard and $t$ - $J$ models," Physical Review $B$, vol. 42, no. 13, pp. 8736-8739, 1990.

[11] D. Poilblanc and E. Dagotto, "Optical mass in the $t-J$ model," Physical Review B, vol. 44, no. 1, pp. 466-469, 1991.

[12] D. Poilblanc, "Twisted boundary conditions in cluster calculations of the optical conductivity in two-dimensional lattice models," Physical Review B, vol. 44, no. 17, pp. 9562-9581, 1991.

[13] E. Dagotto, A. Moreo, F. Ortolani, D. Poilblanc, and J. Riera, "Static and dynamical properties of doped Hubbard clusters," Physical Review B, vol. 45, no. 18, pp. 10741-10760, 1992. 
[14] J.-I. Igarashi and P. Fulde, "Drude weight and optical conductivity of doped antiferromagnets," Physical Review B, vol. 48, no. 17, pp. 12713-12722, 1993.

[15] J. Jaklič and P. Prelovšek, "Finite-temperature conductivity in the planar $t-J$ model," Physical Review B, vol. 50, no. 10, pp. 7129-7132, 1994.

[16] G. Jackeli and N. M. Plakida, "Charge dynamics and optical conductivity of the $t-J$ model," Physical Review B, vol. 60, no. 8, pp. 5266-5275, 1999.

[17] M. Moraghebi, S. Yunoki, and A. Moreo, "Optical conductivity and resistivity of a hole-doped spin-fermion model for cuprates," Physical Review B, vol. 66, no. 21, Article ID 214522, 5 pages, 2002.

[18] A. M. Tikofsky, R. B. Laughlin, and Z. Zou, "Computation of the optical conductivity of the $t-J$ model using anyon techniques," Physical Review Letters, vol. 69, no. 25, pp. 3670 3673, 1992.

[19] N. M. Plakida, "Optical conductivity in the $t$-J model," Zeitschrift fur Physik B, vol. 103, no. 3-4, pp. 383-390, 1997.

[20] R. Eder, P. Wróbel, and Y. Ohta, "Optical conductivity of strongly correlated electron systems," Physical Review B, vol. 54, no. 16, pp. R11034-R11037, 1996.

[21] B. Kyung and S. I. Mukhin, "Dynamics of a small density of holes in a two-dimensional quantum antiferromagnet," Physical Review B, vol. 55, no. 6, pp. 3886-3893, 1997.

[22] E. Dagotto, "Correlated electrons in high-temperature superconductors," Reviews of Modern Physics, vol. 66, no. 3, pp. 763-840, 1994.

[23] N. D. Basov and T. Timusk, "Electrodynamics of high- $T_{C}$ superconductors," Reviews of Modern Physics, vol. 77, no. 2, pp. 721-729, 2005.

[24] M. A. Kastner, R. J. Birgeneau, G. Shirane, and Y. Endoh, "Magnetic, transport, and optical properties of monolayer copper oxides," Reviews of Modern Physics, vol. 70, no. 3, pp. 897-928, 1998.

[25] Y. S. Lee, K. Segawa, Z. Q. Li, et al., "Electrodynamics of the nodal metal state in weakly doped high- $T_{C}$ cuprates," Physical Review B, vol. 72, no. 5, Article ID 054529, 2005.

[26] S. L. Cooper, D. Reznik, A. Kotz, et al., "Optical studies of the $a$-, $b$-, and $c$-axis charge dynamics in $\mathrm{YBa}_{2} \mathrm{Cu}_{3} \mathrm{O}_{6+X}$," Physical Review B, vol. 47, no. 13, pp. 8233-8248, 1993.

[27] G. A. Thomas, D. H. Rapkine, S. L. Cooper, et al., "Optical excitations of a few charges in cuprates," Physical Review B, vol. 45, no. 5, pp. 2474-2479, 1992.

[28] S. Uchida, T. Ido, H. Takagi, T. Arima, Y. Tokura, and S. Tajima, "Optical spectra of $\mathrm{La}_{2-X} \mathrm{Sr}_{X} \mathrm{CuO}_{4}$ : effect of carrier doping on the electronic structure of the $\mathrm{CuO}_{2}$ plane," Physical Review B, vol. 43, no. 10, pp. 7942-7954, 1991.

[29] R. M. A. Azzam and N. M. Bashara, Ellipsometry and Polarized Light, North Holland, New York, NY, USA, 1996.

[30] D. U. Fluckiger, "Analytic methods in the determination of optical properties by spectral ellipsometry," Journal of the Optical Society of America A, vol. 15, no. 8, pp. 2228-2232, 1998.

[31] N. V. Prokof'ev, B. V. Svistunov, and I. S. Tupitsyn, "Exact, complete, and universal continuous-time worldline MonteCarlo approach to the statistics of discrete quantum systems," Zhurnal Eksperimental'noj i Teoreticheskoj Fiziki, vol. 114, no. 2, pp. 570-590, 1998.

[32] N. V. Prokof'ev, B. V. Svistunov, and I. S. Tupitsyn, "Exact, complete, and universal continuous-time worldline Monte Carlo approach to the statistics of discrete quantum systems," Journal of Experimental and Theoretical Physics, vol. 87, no. 2, pp. 310-321, 1998.
[33] N. V. Prokof'ev and B. V. Svistunov, "Polaron problem by diagrammatic quantum Monte Carlo," Physical Review Letters, vol. 81, no. 12, pp. 2514-2517, 1998.

[34] A. S. Mishchenko, N. V. Prokof'ev, A. Sakamoto, and B. V. Svistunov, "Diagrammatic quantum Monte Carlo study of the Fröhlich polaron," Physical Review B, vol. 62, no. 10, pp. 6317-6336, 2000.

[35] A. S. Mishchenko and N. Nagaosa, "Quasidegenerate selftrapping in one-dimensional charge transfer exciton," Physical Review Letters, vol. 86, no. 20, pp. 4624-4627, 2001.

[36] A. S. Mishchenko, N. V. Prokof'ev, B. V. Svistunov, and A. Sakamoto, "Comprehensive study of Fröhlich polaron," International Journal of Modern Physics B, vol. 15, no. 28-30, pp. 3940-3943, 2001.

[37] A. S. Mishchenko, N. V. Prokof'ev, and B. V. Svistunov, "Single-hole spectral function and spin-charge separation in the $t-J$ model," Physical Review B, vol. 64, no. 3, Article ID 033101, 4 pages, 2001.

[38] E. A. Burovski, A. S. Mishchenko, N. V. Prokof'ev, and B. V. Svistunov, "Diagrammatic quantum Monte Carlo for twobody problems: applied to excitons," Physical Review Letters, vol. 87, no. 18, Article ID 186402, 4 pages, 2001.

[39] A. S. Mishchenko, N. Nagaosa, N. V. Prokof'ev, B. V. Svistunov, and E. A. Burovski, "Properties of exciton and exciton-polaron: exact numeric solution," Nonlinear Optics, vol. 29, p. 257, 2002.

[40] A. S. Mishchenko, N. Nagaosa, N. V. Prokof'ev, A. Sakamoto, and B. V. Svistunov, "Self-trapping of polarons in the RashbaPekar model," Physical Review B, vol. 66, no. 2, Article ID 020301, 4 pages, 2002.

[41] A. S. Mishchenko, N. Nagaosa, N. V. Prokof'ev, A. Sakamoto, and B. V. Svistunov, "Optical conductivity of the Fröhlich polaron," Physical Review Letters, vol. 91, no. 23, Article ID 236401, 4 pages, 2003.

[42] A. S. Mishchenko and N. Nagaosa, "Electron-phonon coupling and a polaron in the $t-J$ model: from the weak to the strong coupling regime," Physical Review Letters, vol. 93, no. 3, Article ID 036402, 2004.

[43] A. S. Mishchenko, "Diagrammatic Monte Carlo method as applied to the polaron problems," Uspekhi Fizicheskikh Nauk, vol. 175, no. 9, p. 925, 2005.

[44] A. S. Mishchenko, "Diagrammatic Monte Carlo method as applied to the polaron problems," Physics-Uspekhi, vol. 48, no. 9, pp. 887-902, 2005.

[45] A. S. Mishchenko and N. Nagaosa, "Theory of excitation spectra of electron-phonon coupled systems," Journal of the Physical Society of Japan, vol. 75, no. 1, Article ID 011003, 2006.

[46] A. S. Mishchenko and N. Nagaosa, "Numerical study of the isotope effect in underdoped high-temperature superconductors: calculation of the angle-resolved photoemission spectra," Physical Review B, vol. 73, no. 9, Article ID 092502, 4 pages, 2006.

[47] A. S. Mishchenko and N. Nagaosa, "ARPES spectra of polaron in the $t-J$ model," Journal of Physics and Chemistry of Solids, vol. 67, no. 1-3, pp. 259-261, 2006.

[48] G. De Filippis, V. Cataudella, A. S. Mishchenko, C. A. Perroni, and J. T. Devreese, "Validity of the Franck-Condon principle in the optical spectroscopy: optical conductivity of the Fröhlich polaron," Physical Review Letters, vol. 96, no. 13, Article ID 136405, 4 pages, 2006. 
[49] G. De Filippis, V. Cataudella, A. S. Mishchenko, and N. Nagaosa, "Nonlocal composite spin-lattice polarons in high temperature superconductors," Physical Review Letters, vol. 99, no. 14, Article ID 146405, 2007.

[50] V. Cataudella, G. De Filippis, A. S. Mishchenko, and N. Nagaosa, "Temperature dependence of the angle resolved photoemission spectra in the undoped cuprates: selfconsistent approach to the $t-J$ holstein model," Physical Review Letters, vol. 99, no. 22, Article ID 226402, 2007.

[51] A. S. Mishchenko, N. Nagaosa, Z.-X. Shen, et al., "Charge dynamics of doped holes in high $T_{c}$ cuprate superconductors: a clue from optical conductivity," Physical Review Letters, vol. 100, no. 16, Article ID 166401, 2008.

[52] E. Burovski, H. Fehske, and A. S. Mishchenko, "Exact treatment of exciton-polaron formation by diagrammatic Monte Carlo simulations," Physical Review Letters, vol. 101, no. 11, Article ID 116403, 2008.

[53] A. S. Mishchenko, N. Nagaosa, A. Alvermann, et al., "Localization-delocalization transition of a polaron near an impurity," Physical Review B, vol. 79, no. 18, Article ID 180301, 2009.

[54] A. S. Mishchenko, "Polarons by exact Diagrammatic Monte Carlo and Stochastic optimization method," in Materials and Systems with Reduced Dimensionalities, G. Iadonisi, G. De Filippis, and J. Ranninger, Eds., p. 177, IOS Press, Amsterdam, The Netherlands, 2006.

[55] A. S. Mishchenko and N. Nagaosa, "Spectroscopic properties of polarons in strongly correlated systems by exact diagrammatic Monte Carlo method," in Polarons in Complex Matter, A. S. Alexandrov, Ed., Springer Series in Material Science, pp. 503-544, Springer, New York, NY, USA, 2007.

[56] A. S. Mishchenko, "Diagrammatic Monte Carlo and stochastic optimization methods for complex composite objects in macroscopic baths," in Computational Many-Particle Physics, H. Fehske, R. Schneider, and A. Weisse, Eds., vol. 739 of Lecture Notes in Physics, pp. 367-395, Springer, New York, NY, USA, 2008.

[57] B. O. Wells, Z.-X. Shen, A. Matsuura, et al., " $E$ versus k relations and many body effects in the model insulating copper oxide $\mathrm{Sr}_{2} \mathrm{CuO}_{2} \mathrm{Cl}_{2}$," Physical Review Letters, vol. 74, no. 6, pp. 964-967, 1995.

[58] O. Rösch and O. Gunnarsson, "Dispersion of incoherent spectral features in systems with strong electron-phonon coupling," European Physical Journal B, vol. 43, no. 1, pp. 11$18,2005$.

[59] K. M. Shen, F. Ronning, D. E. Lu, et al., "Missing quasiparticles and the chemical potential puzzle in the doping evolution of the cuprate superconductors," Physical Review Letters, vol. 93, no. 26, Article ID 267002, 2004.

[60] C. Kim, F. Ronning, A. Damascelli, et al., "Anomalous temperature dependence in the photoemission spectral function of cuprates," Physical Review B, vol. 65, no. 17, Article ID 174516, 7 pages, 2002.

[61] O. Rösch and O. Gunnarsson, "Electron-phonon interaction in the $t$-J model," Physical Review Letters, vol. 92, no. 14, Article ID 146403, 2004.

[62] O. Rösch, O. Gunnarsson, X. J. Zhou, et al., "Polaronic behavior of undoped high- $T_{c}$ cuprate superconductors from angleresolved photoemission spectra," Physical Review Letters, vol. 95, no. 22, Article ID 227002, 4 pages, 2005.

[63] S. Kar and E. Manousakis, "Finite-temperature spectral function of a hole in a quantum antiferromagnet and the role of phonons," Physical Review B, vol. 78, no. 6, Article ID 064508, 2008.
[64] A. Lanzara, P. V. Bogdanov, X. J. Zhou, et al., "Evidence for ubiquitous strong electron-phonon coupling in hightemperature superconductors," Nature, vol. 412, no. 6846, pp. 510-514, 2001.

[65] V. J. Emery, "Theory of high- $T_{c}$ superconductivity in oxides," Physical Review Letters, vol. 58, no. 26, pp. 2794-2797, 1987.

[66] F. C. Zhang and T. M. Rice, "Effective Hamiltonian for the superconducting Cu oxides," Physical Review B, vol. 37, no. 7, pp. 3759-3761, 1988.

[67] C. L. Kane, P. A. Lee, and N. Read, "Motion of a single hole in a quantum antiferromagnet," Physical Review B, vol. 39, no. 10, pp. 6880-6897, 1989.

[68] Z. Liu and E. Manousakis, "Dynamical properties of a hole in a Heisenberg antiferromagnet," Physical Review B, vol. 45, no. 5, pp. 2425-2437, 1992.

[69] Z. Liu and E. Manousakis, "Spectral function of a hole in the t-J model," Physical Review B, vol. 44, no. 5, pp. 2414-2417, 1991.

[70] E. Manousakis, "The spin-Heisenberg antiferromagnet on a square lattice and its application to the cuprous oxides," Reviews of Modern Physics, vol. 63, no. 1, pp. 1-62, 1991.

[71] F. Marsiglio, A. E. Ruckenstein, S. Schmitt-Rink, and C. M. Varma, "Spectral function of a single hole in a twodimensional quantum antiferromagnet," Physical Review B, vol. 43, no. 13, Article ID 10882, 1991.

[72] B. Kyung and R. A. Ferrell, "Quasiparticle dispersion of the insulating copper oxide $\mathrm{Sr}_{2} \mathrm{CuO}_{2} \mathrm{Cl}_{2}$ by employing vertical and horizontal double hoppings," Physical Review B, vol. 54, no. 14, pp. 10125-10130, 1996.

[73] T. K. Lee and C. T. Shih, "Dispersion of a single hole in the $t-J$ model," Physical Review B, vol. 55, no. 9, pp. 5983-5987, 1997.

[74] T. Tohyama and S. Maekawa, "Role of next-nearest-neighbor hopping in the $t-t^{\prime}-\mathrm{J}$ model," Physical Review B, vol. 49, no. 5, pp. 3596-3599, 1994.

[75] T. K. Lee, C.-M. Ho, and N. Nagaosa, "Theory for slightly doped antiferromagnetic Mott insulators," Physical Review Letters, vol. 90, no. 6, Article ID 067001, 4 pages, 2003.

[76] T. Tohyama, "Asymmetry of the electronic states in hole- and electron-doped cuprates: exact diagonalization study of the $t-t^{\prime}-t^{\prime \prime}$-J model," Physical Review B, vol. 70, no. 17, Article ID 174517, 12 pages, 2004.

[77] T. Tohyama and S. Maekawa, "Electronic states in the antiferromagnetic phase of electron-doped high- $T_{c}$ cuprates," Physical Review B, vol. 64, no. 21, Article ID 212505, 4 pages, 2001.

[78] T. Tohyama and S. Maekawa, "Doping dependence of chemical potential and entropy in hole- and electron-doped high- $T_{c}$ cuprates," Physical Review B, vol. 67, no. 9, Article ID 092509, 3 pages, 2003.

[79] C. Kim, P. J. White, Z.-X. Shen, et al., "Systematics of the photoemission spectral function of cuprates: insulators and hole- and electron-doped superconductors," Physical Review Letters, vol. 80, no. 19, pp. 4245-4248, 1998.

[80] Yu. A. Izyumov, "The $t-J$ model for strongly correlated electrons and high- $T_{c}$ supercondutors," Uspekhi Fizicheskikh Nauk, vol. 167, no. 5, pp. 496-497, 1997.

[81] Yu. A. Izyumov, "Strongly correlated electrons: the $t-J$ model," Physics-Uspekhi, vol. 40, no. 5, pp. 445-476, 1997.

[82] T. P. Devereaux, A. Virosztek, and A. Zawadowski, "Chargetransfer fluctuation, d-wave superconductivity, and the $B_{1 g}$ Raman phonon in cuprates," Physical Review B, vol. 51, no. 1, pp. 505-514, 1995. 
[83] G. Khaliullin and P. Horsch, "Theory of the density fluctuation spectrum of strongly correlated electrons," Physical Review B, vol. 54, no. 14, pp. R9600-R9603, 1996.

[84] G. Khaliullin and P. Horsch, "Density fluctuations and phonon renormalization in the $t-J$ model," Physica $C$, vol. 282-287, part 3, pp. 1751-1752, 1997.

[85] M. Opel, R. Hackl, T. P. Devereaux, et al., "Physical origin of the buckling in $\mathrm{CuO}_{2}$ : electron-phonon coupling and Raman spectra," Physical Review B, vol. 60, no. 13, pp. 9836-9844, 1999.

[86] T. P. Devereaux, A. Virosztek, and A. Zawadowski, "Neutron scattering and the $B_{1 g}$ phonon in the cuprates," Physical Review B, vol. 59, no. 22, pp. 14618-14623, 1999.

[87] P. Horsch, G. Khaliullin, and V. Oudovenko, "Density response of the $t-J$ model and renormalization of breathing and half-breathing phonon modes: a slave-fermion calculation," Physica C, vol. 341-348, part 1, pp. 117-120, 2000.

[88] S. Ishihara and N. Nagaosa, "Interplay of electron-phonon interaction and electron correlation in high-temperature superconductivity," Physical Review B, vol. 69, no. 14, Article ID 144520, 13 pages, 2004.

[89] O. Rösch and O. Gunnarsson, "Electron-phonon interaction in the three-band model," Physical Review B, vol. 70, no. 22, Article ID 224518, 7 pages, 2004.

[90] T. P. Devereaux, T. Cuk, Z.-X. Shen, and N. Nagaosa, "Anisotropic electron-phonon interaction in the cuprates," Physical Review Letters, vol. 93, no. 11, Article ID 117004, 4 pages, 2004.

[91] T. Cuk, F. Baumberger, D. H. Lu, et al., "Coupling of the $B_{1 g}$ Phonon to the antinodal electronic states of $\mathrm{Bi}_{2} \mathrm{Sr}_{2} \mathrm{Ca}_{0.92} \mathrm{Y}_{0.08} \mathrm{Cu}_{2} \mathrm{O}_{8+\delta}$," Physical Review Letters, vol. 93, no. 11, Article ID 117003, 4 pages, 2004.

[92] P. Horsch and G. Khaliullin, "Doping dependence of density response and bond-stretching phonons in cuprates," Physica B, vol. 359-361, pp. 620-622, 2005.

[93] N. Bulut and D. J. Scalapino, " $d_{x^{2}-y^{2}}$ symmetry and the pairing mechanism," Physical Review B, vol. 54, no. 21, pp. 14971-14973, 1996.

[94] L. Pintschovius and W. Reichardt, "Neutron scattering in layered copper-oxide superconductors," in Physics and Chemistry of Materials with Low-Dimensional Structures, Vol. 20, A. Furrer, Ed., p. 165, Kluwer Academic Publishers, Dordrecht, The Netherlands, 1998.

[95] L. Pintschovius, "Electron-phonon coupling effects explored by inelastic neutron scattering," Physica Status Solidi B, vol. 242, no. 1, pp. 30-50, 2005.

[96] W. Meevasana, T. P. Devereaux, N. Nagaosa, Z.-X. Shen, and J. Zaanen, "Calculation of overdamped $c$-axis charge dynamics and the coupling to polar phonons in cuprate superconductors," Physical Review B, vol. 74, no. 17, Article ID 174524, 6 pages, 2006.

[97] W. Meevasana, N. J. C. Ingle, D. H. Lu, et al., "Doping dependence of the coupling of electrons to bosonic modes in the single-layer high-temperature $\mathrm{Bi}_{2} \mathrm{Sr}_{2} \mathrm{CuO}_{6}$ superconductor," Physical Review Letters, vol. 96, no. 15, Article ID 157003, 4 pages, 2006.

[98] A. S. Alexandrov and P. E. Kornilovitch, "Mobile small polaron," Physical Review Letters, vol. 82, no. 4, pp. 807-810, 1999.

[99] W. E. Pickett, "Electronic structure of the high-temperature oxide superconductors," Review of Modern Physics, vol. 61, pp. 433-512, 1989.
[100] K.-P. Bohnen, R. Heid, and M. Krauss, "Phonon dispersion and electron-phonon interaction for $\mathrm{YBa}_{2} \mathrm{Cu}_{3} \mathrm{O}_{7}$ from firstprinciples calculations," Europhysics Letters, vol. 64, no. 1, pp. 104-110, 2003.

[101] L. Pintschovius and M. Braden, "Anomalous dispersion of LO phonons in $\mathrm{La}_{1.85} \mathrm{Sr}_{0.15} \mathrm{CuO}_{4}$," Physical Review B, vol. 60, no. 22, pp. R15039-R15042, 1999.

[102] F. Giustino, M. L. Cohen, and S. G. Louie, "Small phonon contribution to the photoemission kink in the copper oxide superconductors," Nature, vol. 452, no. 7190, pp. 975-978, 2008.

[103] D. Reznik, G. Sangiovanni, O. Gunnarsson, and T. P. Devereaux, "Photoemission kinks and phonons in cuprates," Nature, vol. 455, no. 7213, pp. E6-E7, 2008.

[104] D. Reznik, L. Pintschovius, M. Ito, et al., "Electron-phonon coupling reflecting dynamic charge inhomogeneity in copper oxide superconductors," Nature, vol. 440, no. 7088, pp. 11701173, 2006.

[105] D. Reznik, L. Pintschovius, M. Fujita, K. Yamada, G. D. Gu, and J. M. Tranquada, "Electron-phonon anomaly related to charge stripes: static stripe phase versus optimally doped superconducting $\mathrm{La}_{1.85} \mathrm{Sr}_{0.15} \mathrm{CuO}_{4}$," Journal of Low Temperature Physics, vol. 147, no. 3-4, pp. 353-364, 2007.

[106] L. Pintschovius, D. Reznik, W. Reichardt, et al., "Oxygen phonon branches in $\mathrm{YBa}_{2} \mathrm{Cu}_{3} \mathrm{O}_{7}$," Physical Review B, vol. 69, no. 21, Article ID 214506, 11 pages, 2004.

[107] H. Uchiyama, A. Q. R. Baron, S. Tsutsui, et al., "Softening of $\mathrm{Cu}-\mathrm{O}$ bond stretching phonons in tetragonal $\mathrm{HgBa}_{2} \mathrm{CuO}_{4+\delta}$," Physical Review Letters, vol. 92, no. 19, Article ID 197005, 4 pages, 2004.

[108] L. Pintschovius, D. Reznik, and K. Yamada, "Oxygen phonon branches in overdoped $\mathrm{La}_{1.7} \mathrm{Sr}_{0.3} \mathrm{Cu}_{3} \mathrm{O}_{4}$," Physical Review $B$, vol. 74, no. 17, Article ID 174514, 5 pages, 2006.

[109] J. J. M. Pothuizen, R. Eder, N. T. Hien, M. Matoba, A. A. Menovsky, and G. A. Sawatzky, "Single hole dynamics in the $\mathrm{CuO}_{2}$ plane at half filling," Physical Review Letters, vol. 78, no. 4, pp. 717-720, 1997.

[110] G.-H. Gweon, T. Sasagawa, S. Y. Zhou, et al., "An unusual isotope effect in a high-transition-temperature superconductor," Nature, vol. 430, no. 6996, pp. 187-190, 2004.

[111] J. F. Douglas, H. Iwasawa, Z. Sun, et al., "Superconductors: unusual oxygen isotope effects in cuprates?" Nature, vol. 446, no. 7133, p. E5, 2007.

[112] H. Iwasawa, Y. Aiura, T. Saitoh, et al., "A re-examination of the oxygen isotope effect in ARPES spectra of Bi2212," Physica C, vol. 463-465, pp. 52-55, 2007.

[113] H. Iwasawa, J. F. Douglas, K. Sato, et al., "Isotopic fingerprint of electron-phonon coupling in high- $T_{c}$ cuprates," Physical Review Letters, vol. 101, no. 15, Article ID 157005, 2008.

[114] J. Van den Brink and O. P. Sushkov, "Single-hole Green's functions in insulating copper oxides at nonzero temperature," Physical Review B, vol. 57, no. 6, pp. 3518-3524, 1998.

[115] S. Lupi, P. Maselli, M. Capizzi, P. Calvani, P. Giura, and P. Roy, "Evolution of a polaron band through the phase diagram of $\mathrm{Nd}_{2-x} \mathrm{Ce}_{x} \mathrm{CuO}_{4-y}$," Physical Review Letters, vol. 83, no. 23, pp. 4852-4855, 1999.

[116] S. Lupi, P. Calvani, M. Capizzi, P. Maselli, W. Sadowski, and E. Walker, "Infrared optical conductivity of the $\mathrm{Nd}-\mathrm{Ce}-\mathrm{Cu}-\mathrm{O}$ system," Physical Review B, vol. 45, no. 21, pp. 12470-12477, 1992.

[117] S. Lupi, M. Capizzi, P. Calvani, et al., "Fano effect in the $a-b$ plane of $\mathrm{Nd}_{1.96} \mathrm{Ce}_{0.04} \mathrm{CuO}_{4+y}$ : evidence of phonon interaction with a polaronic background," Physical Review B, vol. 57, no. 2, pp. 1248-1252, 1998. 
[118] M. A. Quijada, D. B. Tanner, R. J. Kelley, M. Onellion, H. Berger, and G. Margaritondo, "Anisotropy in the ab-plane optical properties of $\mathrm{Bi}_{2} \mathrm{Sr}_{2} \mathrm{CaCu}_{2} \mathrm{O}_{8}$ single-domain crystals," Physical Review B, vol. 60, no. 21, pp. 14917-14934, 1999.

[119] Y. Onose, Y. Taguchi, K. Ishizaka, and Y. Tokura, "Doping dependence of pseudogap and related charge dynamics in $\mathrm{Nd}_{2-x} \mathrm{Ce}_{x} \mathrm{CuO}_{4}, "$ Physical Review Letters, vol. 87, no. 21, Article ID 217001, 4 pages, 2001.

[120] N. L. Wang, P. Zheng, T. Feng, et al., "Infrared properties of $\mathrm{La}_{2-x}(\mathrm{Ca}, \mathrm{Sr})_{x} \mathrm{CaCu}_{2} \mathrm{O}_{6+\delta}$ single crystals," Physical Review B, vol. 67, no. 13, Article ID 134526, 5 pages, 2003.

[121] P. D. Johnson, T. Valla, A. V. Fedorov, et al., "Doping and temperature dependence of the mass enhancement observed in the cuprate $\mathrm{Bi}_{2} \mathrm{Sr}_{2} \mathrm{CaCu}_{2} \mathrm{O}_{8+\delta}$," Physical Review Letters, vol. 87, no. 17, Article ID 86394, 1770074 pages, 2001.

[122] T. Valla, A. V. Fedorov, P. D. Johnson, et al., "Evidence for quantum critical behavior in the optimally doped cuprate $\mathrm{Bi}_{2} \mathrm{Sr}_{2} \mathrm{CaCu}_{2} \mathrm{O}_{8+\delta}$," Science, vol. 285, no. 5436, pp. 2110 2113, 1999.

[123] A. Kaminski, J. Mesot, H. Fretwell, et al., "Quasiparticles in the superconducting state of $\mathrm{Bi}_{2} \mathrm{Sr}_{2} \mathrm{CaCu}_{2} \mathrm{O}_{8+\delta}$," Physical Review Letters, vol. 84, no. 8, pp. 1788-1791, 2000.

[124] P. V. Bogdanov, A. Lanzara, S. A. Kellar, et al., "Evidence for an energy scale for quasiparticle dispersion in $\mathrm{Bi}_{2} \mathrm{Sr}_{2} \mathrm{CaCu}_{2} \mathrm{O}_{8}$," Physical Review Letters, vol. 85, no. 12, pp. 2581-2584, 2000.

[125] A. Kaminski, M. Randeria, J. C. Campuzano, et al., "Renormalization of spectral line shape and dispersion below $T_{c}$ in $\mathrm{Bi}_{2} \mathrm{Sr}_{2} \mathrm{CaCu}_{2} \mathrm{O}_{8+\delta}$," Physical Review Letters, vol. 86, no. 6, pp. 1070-1073, 2001.

[126] A. D. Gromko, A. V. Fedorov, Y.-D. Chuang, et al., "Massrenormalized electronic excitations at $(\pi, 0)$ in the superconducting state of $\mathrm{Bi}_{2} \mathrm{Sr}_{2} \mathrm{CaCu}_{2} \mathrm{O}_{8+\delta}$," Physical Review $B$, vol. 68, no. 17, Article ID 174520, 2003.

[127] T. Sato, H. Matsui, T. Takahashi, et al., "Observation of band renormalization effects in hole-doped high- $T_{c}$ superconductors," Physical Review Letters, vol. 91, no. 15, Article ID 157003, 2003.

[128] X. J. Zhou, J. Shi, T. Yoshida, et al., "Multiple bosonic mode coupling in the electron self-energy of $\left(\mathrm{La}_{2-x} \mathrm{Sr}_{x}\right) \mathrm{CuO}_{4}$," Physical Review Letters, vol. 95, no. 11, Article ID 117001, 4 pages, 2005.

[129] M. R. Norman, H. Ding, J. C. Campuzano, et al., "Unusual dispersion and line shape of the superconducting state spectra of $\mathrm{Bi}_{2} \mathrm{Sr}_{2} \mathrm{CaCu}_{2} \mathrm{O}_{8+\delta}$," Physical Review Letters, vol. 79, no. 18, pp. 3506-3509, 1997.

[130] T. K. Kim, A. A. Kordyuk, S. V. Borisenko, et al., "Doping dependence of the mass enhancement in $(\mathrm{Pb}, \mathrm{Bi})_{2} \mathrm{Sr}_{2} \mathrm{CaCu}_{2} \mathrm{O}_{8}$ at the antinodal point in the superconducting and normal states," Physical Review Letters, vol. 91, no. 16, Article ID 167002, 4 pages, 2003.

[131] R. Zeyher and A. Greco, "Low-energy renormalization of the electron dispersion of high- $T_{c}$ superconductors," Physical Review B, vol. 64, no. 14, Article ID 140510, 4 pages, 2001.

[132] S. Koikegami and Y. Aiura, "Kink structure in the electronic dispersion of high- $T_{c}$ superconductors from the electronphonon interaction," Physical Review B, vol. 77, no. 18, Article ID 184519, 2008.

[133] M. R. Norman and H. Ding, "Collective modes and the superconducting-state spectral function of $\mathrm{Bi}_{2} \mathrm{Sr}_{2} \mathrm{CaCu}_{2} \mathrm{O}_{8}$," Physical Review B, vol. 57, no. 18, pp. R11089-R11092, 1998.

[134] M. Eschrig and M. R. Norman, "Neutron resonance: modeling photoemission and tunneling data in the superconduct- ing state of $\mathrm{Bi}_{2} \mathrm{Sr}_{2} \mathrm{CaCu}_{2} \mathrm{O}_{8+\delta}$," Physical Review Letters, vol. 85, no. 15, pp. 3261-3264, 2000.

[135] D. Manske, I. Eremin, and K. H. Bennemann, "Analysis of the elementary excitations in high- $T_{c}$ cuprates: explanation of the new energy scale observed by angle-resolved photoemission spectroscopy," Physical Review Letters, vol. 87, no. 17, Article ID 177005, 4 pages, 2001.

[136] M. Eschrig and M. R. Norman, "Dispersion anomalies in bilayer cuprates and the odd symmetry of the magnetic resonance," Physical Review Letters, vol. 89, no. 27, Article ID 277005, 4 pages, 2002.

[137] D. Manske, I. Eremin, and K. H. Bennemann, "Renormalization of the elementary excitations in hole- and electrondoped cuprates due to spin fluctuations," Physical Review B, vol. 67, no. 13, Article ID 134520, 12 pages, 2003.

[138] A. V. Chubukov and M. R. Norman, "Dispersion anomalies in cuprate superconductors," Physical Review B, vol. 70, no. 17, Article ID 174505, 12 pages, 2004.

[139] D. Manske, Theory of Unconventional Superconductors: Cooper Pairing Mediated by Spin Excitations, vol. 202 of Springer Tracts in Modern Pysics, Springer, Heidelberg, Germany, 2004.

[140] H. F. Fong, P. Bourges, Y. Sidis, et al., "Neutron scattering from magnetic excitations in $\mathrm{Bi}_{2} \mathrm{Sr}_{2} \mathrm{CaCu}_{2} \mathrm{O}_{8+\delta}$," Nature, vol. 398, no. 6728, pp. 588-591, 1999.

[141] H. A. Mook, M. Yethiraj, G. Aeppli, T. E. Mason, and T. Armstrong, "Polarized neutron determination of the magnetic excitations in $\mathrm{YBa}_{2} \mathrm{Cu}_{3} \mathrm{O}_{7}$," Physical Review Letters, vol. 70, no. 22, pp. 3490-3493, 1993.

[142] H. F. Fong, B. Keimer, P. W. Anderson, D. Reznik, F. Doğan, and I. A. Aksay, "Phonon and magnetic neutron scattering at $41 \mathrm{meV}$ in $\mathrm{YBa}_{2} \mathrm{Cu}_{3} \mathrm{O}_{7}$," Physical Review Letters, vol. 75, no. 2, pp. 316-319, 1995.

[143] B. Vignolle, S. M. Hayden, D. F. McMorrow, et al., "Two energy scales in the spin excitations of the high-temperature superconductor $\mathrm{La}_{2-x} \mathrm{Sr}_{x} \mathrm{CuO}_{4}$," Nature Physics, vol. 3, no. 3, pp. 163-167, 2007.

[144] A. W. Sandvik, D. J. Scalapino, and N. E. Bickers, "Effect of an electron-phonon interaction on the one-electron spectral weight of a $d$-wave superconductor," Physical Review B, vol. 69, no. 9, Article ID 094523, 11 pages, 2004.

[145] S. R. Park, D. J. Song, C. S. Leem, et al., "Angle-resolved photoemission spectroscopy of electron-doped cuprate superconductors: isotropic electron-phonon coupling," Physical Review Letters, vol. 101, no. 11, Article ID 117006, 4 pages, 2008.

[146] D. Reznik, B. Keimer, F. Dogan, and I. A. Aksay, "q dependence of self-energy effects of the plane oxygen vibration in $\mathrm{YBa}_{2} \mathrm{Cu}_{3} \mathrm{O}_{7}$," Physical Review Letters, vol. 75, no. 12, pp. 2396-2399, 1995.

[147] R. J. McQueeney, Y. Petrov, T. Egami, M. Yethiraj, G. Shirane, and Y. Endoh, "Anomalous dispersion of LO phonons in $\mathrm{La}_{1.85} \mathrm{Sr}_{0.15} \mathrm{CuO}_{4}$ at low temperatures," Physical Review Letters, vol. 82, no. 3, pp. 628-631, 1999.

[148] P. Dai, H. A. Mook, S. M. Hayden, et al., "The magnetic excitation spectrum and thermodynamics of high- $T_{c}$ superconductors," Science, vol. 284, no. 5418, pp. 1344-1347, 1999.

[149] S. D. Wilson, P. Dai, S. Li, S. Chi, H. J. Kang, and J. W. Lynn, "Resonance in the electron-doped high-transitiontemperature superconductor $\mathrm{PrLaCe}_{0.12} \mathrm{CuO}_{4-\delta}$," Nature, vol. 442, no. 7098, pp. 59-62, 2006.

[150] J. Zhao, P. Dai, S. Li, P. G. Freeman, Y. Onose, and Y. Tokura, "Neutron-spin resonance in the optimally electron-doped 
superconductor $\mathrm{Nd}_{1.85} \mathrm{Ce}_{0.15} \mathrm{CuO}_{4-\delta}$," Physical Review Letters, vol. 99, no. 1, Article ID 017001, 4 pages, 2007.

[151] L. Pintschovius, N. Pyka, W. Reichardt, et al., "Lattice dynamical studies of HTSC materials," Physica C, vol. 185189, pp. 156-161, 1991.

[152] L. Pintschovius and M. Braden, "Phonon anomalies in $\mathrm{La}_{2} \mathrm{CuO}_{4-\delta}$," Journal of Low Temperature Physics, vol. 105, no. 3-4, pp. 813-818, 1996.

[153] W. Reichardt, "Cu-O bond-stretching vibrations in $\mathrm{YBa}_{2} \mathrm{Cu}_{3} \mathrm{O}_{7}$ studied by inelastic neutron scattering," Journal of Low Temperature Physics, vol. 105, no. 3-4, pp. 807-812, 1996.

[154] R. J. McQueeney, J. L. Sarrao, P. G. Pagliuso, P. W. Stephens, and R. Osborn, "Mixed lattice and electronic states in hightemperature superconductors," Physical Review Letters, vol. 87, no. 7, Article ID 077001, 4 pages, 2001.

[155] L. Pintschovius, W. Reichardt, M. Kläser, T. Wolf, and H. V. Löhneysen, "Pronounced in-plane anisotropy of phonon anomalies in $\mathrm{YBa}_{2} \mathrm{Cu}_{3} \mathrm{O}_{6.6}$," Physical Review Letters, vol. 89, no. 3, Article ID 037001, 4 pages, 2002.

[156] J.-H. Chung, T. Egami, R. J. McQueeney, et al., "In-plane anisotropy and temperature dependence of oxygen phonon modes in $\mathrm{YBa}_{2} \mathrm{Cu}_{3} \mathrm{O}_{6.95}$," Physical Review $B$, vol. 67, no. 1, Article ID 014517, 9 pages, 2003.

[157] N. Pyka, W. Reichardt, L. Pintschovius, G. Engel, J. Rossat-Mignod, and J. Y. Henry, "Superconductivity-induced phonon softening in $\mathrm{YBa}_{2} \mathrm{Cu}_{3} \mathrm{O}_{7}$ observed by inelastic neutron scattering," Physical Review Letters, vol. 70, no. 10, pp. 1457-1460, 1993.

[158] S. L. Cooper, M. V. Klein, B. G. Pazol, J. P. Rice, and D. M. Ginsberg, "Raman scattering from superconducting gap excitations in single-crystal $\mathrm{YBa}_{2} \mathrm{Cu}_{3} \mathrm{O}_{7-\delta}$," Physical Review $B$, vol. 37, no. 10, pp. 5920-5923, 1988.

[159] C. Thomsen, M. Cardona, B. Gegenheimer, R. Liu, and A. Simon, "Untwinned single crystals of $\mathrm{YBa}_{2} \mathrm{Cu}_{3} \mathrm{O}_{7--}$ an optical investigation of the a-b anisotropy," Physical Review B, vol. 37, no. 16, pp. 9860-9863, 1988.

[160] J. Graf, M. D’Astuto, P. Giura, et al., "In-plane copperoxygen bond-stretching mode anomaly in underdoped $\mathrm{La}_{2-x} \mathrm{Sr}_{x} \mathrm{CuO}_{4+\delta}$ measured with high-resolution inelastic Xray scattering," Physical Review B, vol. 76, no. 17, Article ID 172507, 2007.

[161] J. Graf, M. D’Astuto, C. Jozwiak, et al., "Bond stretching phonon softening and kinks in the angle-resolved photoemission spectra of optimally doped $\mathrm{Bi}_{2} \mathrm{Sr}_{1.6} \mathrm{La}_{0.4} \mathrm{Cu}_{2} \mathrm{O}_{6+\delta}$ superconductors," Physical Review Letters, vol. 100, no. 22, Article ID 227002, 2008.

[162] K. J. von Szczepanski and K. W. Becker, "Coupling of electrons and phonons in a doped antiferromagnet," Zeitschrift für Physik B, vol. 89, no. 3, pp. 327-334, 1992.

[163] C. Falter and M. Klenner, "Nonadiabatic and nonlocal electron-phonon interaction and phonon-plasmon mixing in the high-temperature superconductors," Physical Review B, vol. 50, no. 13, pp. 9426-9433, 1994.

[164] C. Falter, M. Klenner, and G. A. Hoffmann, "Phonon renormalization and c-axis phonon-plasmon mixing in $\mathrm{La}_{2} \mathrm{CuO}_{4}$," Physical Review B, vol. 52, no. 5, pp. 3702-3710, 1995.

[165] B. Bäuml, G. Wellein, and H. Fehske, "Optical absorption and single-particle excitations in the two-dimensional Holstein t-J model," Physical Review B, vol. 58, no. 7, pp. 3663-3676, 1998.
[166] A. Ramak, P. Horsch, and P. Fulde, "Effective mass of quasiparticles in a $t-J$ model with electron-phonon interactions," Physical Review B, vol. 46, no. 21, pp. 14305-14308, 1992.

[167] B. Kyung, S. I. Mukhin, V. N. Kostur, and R. A. Ferrell, "Spectral properties of the $t-J$ model in the presence of holephonon interaction," Physical Review B, vol. 54, no. 18, pp. 13167-13174, 1996.

[168] H. Röder, H. Fehske, and R. N. Silver, "The ordering of polarons in the Holstein $t-J$ model: an application to $\mathrm{La}_{2-x} \mathrm{Sr}_{x} \mathrm{NiO}_{4+y}$," Europhysics Letters, vol. 28, no. 4, pp. 257262, 1994.

[169] S. Schmitt-Rink, C. M. Varma, and A. E. Ruckenstein, "Spectral function of holes in a quantum antiferromagnet," Physical Review Letters, vol. 60, no. 26, pp. 2793-2796, 1988.

[170] G. Martinez and P. Horsch, "Spin polarons in the $t$-J model," Physical Review B, vol. 44, no. 1, pp. 317-331, 1991.

[171] O. Gunnarsson and O. Rösch, "Electron-phonon coupling in the self-consistent Born approximation of the $t-J$ model," Physical Review B, vol. 73, no. 17, Article ID 174521, 2006.

[172] J. Bonča, S. Maekawa, T. Tohyama, and P. Prelovšek, "Spectral properties of a hole coupled to optical phonons in the generalized $t$-J model," Physical Review B, vol. 77, no. 5, Article ID 054519, 2008.

[173] J. Bonča, S. A. Trugman, and I. Batistić, "Holstein polaron," Physical Review B, vol. 60, no. 3, pp. 1633-1642, 1999.

[174] J. Bonča, S. Maekawa, and T. Tohyama, "Numerical approach to the low-doping regime of the $t-J$ model," Physical Review $B$, vol. 76, no. 3, Article ID 035121, 6 pages, 2007.

[175] E. I. Rashba, "Self-trapping of excitons," in Modern Problems in Condensed Matter Sciences, V. M. Agranovich and A. A. Maradudin, Eds., vol. 2, p. 543, Notrh Holland, Amsterdam, The Netherlands, 1982.

[176] A. S. Ioselevich and E. I. Rashba, "Theory of nonradiative trapping in crystals," in Modern Problems in Condensed Matter Sciences, vol. 34, p. 347, Notrh Holland, Amsterdam, The Netherlands, 1992.

[177] K. M. Shen, F. Ronning, W. Meevasana, et al., "Angle-resolved photoemission studies of lattice polaron formation in the cuprate $\mathrm{Ca}_{2} \mathrm{CuO}_{2} \mathrm{Cl}_{2}$," Physical Review B, vol. 75, no. 7, Article ID 075115, 2007.

[178] F. Ronning, K. M. Shen, N. P. Armitage, et al., "Anomalous high-energy dispersion in angle-resolved photoemission spectra from the insulating cuprate $\mathrm{Ca}_{2} \mathrm{CuO}_{2} \mathrm{Cl}_{2}$," Physical Review B, vol. 71, no. 9, Article ID 094518, 5 pages, 2005.

[179] J. Graf, G.-H. Gweon, K. McElroy, et al., "Universal high energy anomaly in the angle-resolved photoemission spectra of high temperature superconductors: possible evidence of spinon and holon branches," Physical Review Letters, vol. 98, no. 6, Article ID 067004, 2007.

[180] B. P. Xie, K. Yang, D. W. Shen, et al., "High-energy scale revival and giant kink in the dispersion of a cuprate superconductor," Physical Review Letters, vol. 98, no. 14, Article ID 147001, 2007.

[181] T. Valla, T. E. Kidd, W.-G. Yin, et al., "High-energy kink observed in the electron dispersion of high-temperature cuprate superconductors," Physical Review Letters, vol. 98, no. 16, Article ID 167003, 2007.

[182] W. Meevasana, X. J. Zhou, S. Sahrakorpi, et al., "Hierarchy of multiple many-body interaction scales in high-temperature superconductors," Physical Review B, vol. 75, no. 17, Article ID 174506, 2007.

[183] E. Manousakis, "String excitations of a hole in a quantum antiferromagnet and photoelectron spectroscopy," Physical Review B, vol. 75, no. 3, Article ID 035106, 2007. 
[184] E. Manousakis, "Possible observation of "string excitations" of a hole in a quantum antiferromagnet," Physics Letters A, vol. 362, no. 1, pp. 86-89, 2007.

[185] F. Carbone, D.-S. Yang, E. Giannini, and A. H. Zewail, "Direct role of structural dynamics in electron-lattice coupling of superconducting cuprates," Proceedings of the National Academy of Sciences of the United States of America, vol. 105, no. 51, pp. 20161-20166, 2008.

[186] P. B. Allen, "Theory of thermal relaxation of electrons in metals," Physical Review Letters, vol. 59, no. 13, pp. 1460$1463,1987$.

[187] M. Grüninger, D. van der Marel, A. Damascelli, A. Erb, T. Nunner, and T. Kopp, "Midinfrared absorption in $\mathrm{YBa}_{2} \mathrm{Cu}_{3} \mathrm{O}_{6}$ : evidence for a failure of spin-wave theory for spin 1/2 in two dimensions," Physical Review B, vol. 62, no. 18, pp. 12422-12426, 2000.

[188] I. Eremin, O. Kamaev, and M. V. Eremin, "Possible isotope effect on the resonance peak formation in high- $T_{c}$ cuprates," Physical Review B, vol. 69, no. 9, Article ID 094517, 5 pages, 2004.

[189] G.-M. Zhao, "Evidence for very strong electron-phonon coupling in $\mathrm{YBa}_{2} \mathrm{Cu}_{3} \mathrm{O}_{6}$," Physical Review B, vol. 75, no. 10, Article ID 104511, 2007.

[190] G.-H. Gweon, S. Y. Zhou, M. C. Watson, T. Sasagawa, H. Takagi, and A. Lanzara, "Strong and complex electron-lattice correlation in optimally doped $\mathrm{Bi}_{2} \mathrm{Sr}_{2} \mathrm{CaCu}_{2} \mathrm{O}_{8+\delta}$," Physical Review Letters, vol. 97, no. 22, Article ID 227001, 2006.

[191] J. Lee, K. Fujita, K. McElroy, et al., "Interplay of electrong-lattice interactions and superconductivity in $\mathrm{Bi}_{2} \mathrm{Sr}_{2} \mathrm{CaCu}_{2} \mathrm{O}_{8+\delta}$," Nature, vol. 442, no. 7102, pp. 546-550, 2006.

[192] A. De Lozanne, "Superconductivity: hot vibes," Nature, vol. 442, no. 7102, pp. 522-523, 2006.

[193] J. D. Koralek, J. F. Douglas, N. C. Plumb, et al., "Laser based angle-resolved photoemission, the sudden approximation, and quasiparticle-like spectral peaks in $\mathrm{Bi}_{2} \mathrm{Sr}_{2} \mathrm{CaCu}_{2} \mathrm{O}_{8+\delta}$," Physical Review Letters, vol. 96, no. 1, Article ID 017005, 2006.

[194] J. P. Hague, P. E. Kornilovitch, A. S. Alexandrov, and J. H. Samson, "Effects of lattice geometry and interaction range on polaron dynamics," Physical Review B, vol. 73, no. 5, Article ID 054303, 14 pages, 2006.

[195] P. E. Spencer, J. H. Samson, P. E. Kornilovitch, and A. S. Alexandrov, "Effect of electron-phonon interaction range on lattice polaron dynamics: a continuous-time quantum Monte Carlo study," Physical Review B, vol. 71, no. 18, Article ID 184310, 2005.

[196] P. E. Kornilovitch and A. S. Alexandrov, "Isotope effect on the electron band structure of doped insulators," Physical Review $B$, vol. 70, no. 22, Article ID 224511, 2004.

[197] P. E. Kornilovitch, "Giant enhancement of anisotropy by electron-phonon interaction," Physical Review B, vol. 59, no. 21, pp. 13531-13534, 1999.

[198] P. E. Kornilovitch, "Continuous-time quantum Monte Carlo algorithm for the lattice polaron," Physical Review Letters, vol. 81, no. 24, pp. 5382-5385, 1998.

[199] P. Paci, M. Capone, E. Cappelluti, S. Ciuchi, C. Grimaldi, and L. Pietronero, "Polaronic and nonadiabatic phase diagram from anomalous isotope effects," Physical Review Letters, vol. 94, no. 3, Article ID 036406, 4 pages, 2005.

[200] S. Andergassen, S. Caprara, C. Di Castro, and M. Grilli, "Anomalous isotopic effect near the charge-ordering quantum criticality," Physical Review Letters, vol. 87, no. 5, Article ID 056401, 4 pages, 2001.
[201] S. Fratini and S. Ciuchi, "Spectral properties and isotope effect in strongly interacting systems: Mott-Hubbard insulator versus polaronic semiconductor," Physical Review B, vol. 72, no. 23, Article ID 235107, 9 pages, 2005.

[202] P. Paci, M. Capone, E. Cappelluti, S. Ciuchi, and C. Grimaldi, "Isotope effects in the Hubbard-Holstein model within dynamical mean-field theory," Physical Review B, vol. 74, no. 20, Article ID 205108, 2006.

[203] G. A. Sawatzky, “Testing Fermi-liquid models," Nature, vol. 342, no. 6249, pp. 480-481, 1989.

[204] D. S. Dessau, T. Saitoh, C.-H. Park, et al., "k-dependent electronic structure, a large "ghost" fermi surface, and a pseudogap in a layered magnetoresistive oxide," Physical Review Letters, vol. 81, no. 1, pp. 192-195, 1998.

[205] L. Perfetti, H. Berger, A. Reginelli, et al., "Spectroscopic indications of polaronic carriers in the quasi-one-dimensional conductor $\left(\mathrm{TaSe}_{4}\right)_{2} \mathrm{I}$," Physical Review Letters, vol. 87, no. 21, Article ID 216404, 2001.

[206] L. Perfetti, S. Mitrovic, G. Margaritondo, et al., "Mobile small polarons and the Peierls transition in the quasi-onedimensional conductor $\mathrm{K}_{0.3} \mathrm{MoO}_{3}$," Physical Review B, vol. 66, no. 7, Article ID 075107, 8 pages, 2002.

[207] D. Schrupp, M. Sing, M. Tsunekawa, et al., "High-energy photoemission on $\mathrm{Fe}_{3} \mathrm{O}_{4}$ : small polaron physics and the Verwey transition," Europhysics Letters, vol. 70, no. 6, pp. 789795, 2005.

[208] G. D. Mahan, Many Particle Physics, Plenum Press, New York, NY, USA, 2000.

[209] H. Röder, H. Fehske, and H. Büttner, "Exact diagonalization study of the two-dimensional $t$ - $J$ model with adiabatic Holstein phonons: single-hole case," Physical Review B, vol. 47, no. 19, pp. 12420-12425, 1993.

[210] P. Prelovšek, R. Zeyher, and P. Horsch, "Self-localization of composite spin-lattice polarons," Physical Review Letters, vol. 96, no. 8, Article ID 086402, 4 pages, 2006.

[211] V. Cataudella, G. De Filippis, A. S. Mishchenko, and N. Nagaosa, "Evidences of the charge-lattice interaction in undoped cuprates," Journal of Superconductivity and Novel Magnetism, vol. 22, no. 1, pp. 17-20, 2009.

[212] M. Berciu, “Green's function of a dressed particle," Physical Review Letters, vol. 97, no. 3, Article ID 036402, 2006.

[213] G. L. Goodvin, M. Berciu, and G. A. Sawatzky, "Green's function of the Holstein polaron," Physical Review B, vol. 74, no. 24, Article ID 245104, 2006.

[214] M. Berciu, "Berciu Replies-comment on “Green's function of a dressed particle"'” Physical Review Letters, vol. 98, no. 20, Article ID 209702, 1 pages, 2007.

[215] M. Berciu and G. L. Goodvin, "Systematic improvement of the momentum average approximation for the Green's function of a Holstein polaron," Physical Review B, vol. 76, no. 16, Article ID 165109, 2007.

[216] L. Covaci and M. Berciu, "Holstein polaron: the effect of coupling to multiple-phonon modes," Europhysics Letters, vol. 80, no. 6, Article ID 67001, 2007.

[217] L. Covaci and M. Berciu, "Survival of the dirac points in rippled graphene," Physical Review Letters, vol. 100, no. 25, Article ID 256405, 2008.

[218] G. L. Goodvin and M. Berciu, "Momentum average approximation for models with electron-phonon coupling dependent on the phonon momentum," Physical Review B, vol. 78, no. 23, Article ID 235120, 2008.

[219] L. Covaci and M. Berciu, "Polaron formation in the presence of Rashba spin-orbit coupling: implications for spintronics," 
Physical Review Letters, vol. 102, no. 18, Article ID 186403, 2009.

[220] O. S. Barišić, “Comment: Green's function of a dressed particle," Physical Review Letters, vol. 98, no. 20, Article ID 209701, 2007.

[221] O. S. Barišić, "Diagrammatic content of the dynamical meanfield theory for the Holstein polaron problem in finite dimensions," Physical Review B, vol. 76, no. 19, Article ID 193106, 2007.

[222] J. K. Freericks, M. Jarrell, and D. J. Scalapino, "Holstein model in infinite dimensions," Physical Review B, vol. 48, no. 9, pp. 6302-6314, 1993.

[223] E. Cappelluti and S. Ciuchi, "Magnetic and lattice polaron in the Holstein $t-J$ model," Physical Review B, vol. 66, no. 16, Article ID 165102, 12 pages, 2002.

[224] E. Cappelluti, S. Ciuchi, and S. Fratini, "Polaronic features in the optical properties of the Holstein $t-J$ model," Physical Review B, vol. 76, no. 12, Article ID 125111, 2007.

[225] M. Cini and A. D. Andrea, "Exactly solved electron-boson models in condensed matter and molecular physics by a generalised recursion method," Journal of Physics C, vol. 21, pp. 193-235, 1988.

[226] S. Cuichi, et al., "Dynamical mean-field theory of the small polaron," Physical Review B, vol. 56, p. 4494, 1997.

[227] A. J. Millis, R. Mueller, and B. I. Shraiman, "Fermi-liquid-topolaron crossover. I. General results," Physical Review B, vol. 54, no. 8, pp. 5389-5404, 1996.

[228] P. Benedetti and R. Zeyher, "Holstein model in infinite dimensions at half-filling," Physical Review B, vol. 58, no. 21, pp. 14320-14334, 1998.

[229] D. Meyer, A. C. Hewson, and R. Bulla, "Gap formation and soft phonon mode in the Holstein model," Physical Review Letters, vol. 89, no. 19, Article ID 196401, 4 pages, 2002.

[230] J. E. Han, O. Gunnarsson, and V. H. Crespi, "Strong superconductivity with local Jahn-Teller phonons in $\mathrm{C}_{60}$ solids," Physical Review Letters, vol. 90, no. 16, Article ID 167006, 4 pages, 2003.

[231] M. Capone and S. Ciuchi, "Polaron crossover and bipolaronic metal-insulator transition in the half-filled Holstein model," Physical Review Letters, vol. 91, no. 18, Article ID 186405, 4 pages, 2003.

[232] M. Capone, P. Carta, and S. Ciuchi, "Dynamical mean field theory of polarons and bipolarons in the half-filled Holstein model," Physical Review B, vol. 74, no. 4, Article ID 045106, 2006.

[233] H. Barentzen, "Intermediate-coupling theory of the spin polaron in the $t-J$ model," Physical Review B, vol. 53, no. 9, pp. 5598-5608, 1996.

[234] S. Yunoki, A. Macridin, and G. Sawatzky, private communication.

[235] J. J. M. Pothuizen, Ph.D. thesis, Groningen, The Netherlands, 1998.

[236] C. Kim, private communication.

[237] Z. Liu and E. Manousakis, "Loop-expansion study of the single-hole spectral function in the $t-J$ model," Physical Review B, vol. 51, no. 5, pp. 3156-3162, 1995.

[238] A. S. Alexandrov and K. Reynolds, "Angle-resolved photoemission spectroscopy of band tails in lightly doped cuprates," Physical Review B, vol. 76, no. 13, Article ID 132506, 4 pages, 2007.

[239] G. De Filippis, V. Cataudella, and G. Iadonisi, "Normal state properties of an interacting large polaron gas," European Physical Journal B, vol. 8, no. 3, pp. 339-351, 1999.
[240] J. Lorenzana, "Instability due to long-range Coulomb interaction in a liquid of Feynman polarons," Europhysics Letters, vol. 53, no. 4, pp. 532-538, 2001.

[241] J. Tempere and J. T. Devreese, "Sum rule for the optical absorption of an interacting many-polaron gas," European Physical Journal B, vol. 20, no. 1, pp. 27-33, 2001.

[242] J. Tempere and J. T. Devreese, "Optical absorption of an interacting many-polaron gas," Physical Review B, vol. 64, no. 10, Article ID 104504, 10 pages, 2001.

[243] E. Cappelluti, S. Ciuchi, and S. Fratini, "Polaronic signatures in the optical properties of the electron-doped cuprate superconductor $\mathrm{Nd}_{2-x} \mathrm{Ce}_{x} \mathrm{CuO}_{4}$, " Physical Review B, vol. 79, no. 1, Article ID 012502, 4 pages, 2009.

[244] R. Liang, D. A. Bonn, and W. N. Hardy, "Evaluation of $\mathrm{CuO}_{2}$ plane hole doping in $\mathrm{YBa}_{2} \mathrm{Cu}_{3} \mathrm{O}_{6+x}$ single crystals," Physical Review B, vol. 73, no. 18, Article ID 180505(R), 4 pages, 2006.

[245] D. Mihailović, C. M. Foster, K. Voss, and A. J. Heeger, "Application of the polaron-transport theory to $\sigma(\omega)$ in $\mathrm{Tl}_{2} \mathrm{Ba}_{2} \mathrm{Ca}_{1-x} \mathrm{Gd}_{x} \mathrm{Cu}_{2} \mathrm{O}_{8}, \mathrm{YBa}_{2} \mathrm{Cu}_{3} \mathrm{O}_{7-\delta}$, and $\mathrm{La}_{2-x} \mathrm{Sr}_{x} \mathrm{CuO}_{4}$," Physical Review B, vol. 42, no. 13, pp. 7989-7993, 1990.

[246] X.-X. Bi and P. C. Eklund, "Polaron contribution to the infrared optical response of $\mathrm{La}_{2-x} \mathrm{Sr}_{x} \mathrm{CuO}_{4+\delta}$ and $\mathrm{La}_{2-x} \mathrm{Sr}_{x} \mathrm{NiO}_{4+\delta}$, Physical Review Letters, vol. 70, no. 17, pp. 2625-2628, 1993.

[247] A. S. Alexandrov and N. Mott, Polarons and Bipolarons, World Scientific, Singapore, 1995.

[248] J. T. Devreese and J. Tempere, "Large-polaron effects in the infrared spectrum of high- $T_{c}$ cuprate superconductors," Solid State Communications, vol. 106, no. 5, pp. 309-313, 1998.

[249] J. T. Devreese, "Fröhlich polarons from 0D to 3D: concepts and recent developments," Journal of Physics: Condensed Matter, vol. 19, no. 25, Article ID 255201, 23 pages, 2007.

[250] A. E. Myasnikova and E. N. Myasnikov, "Correlation of optical conductivity and angle-resolved photoemission spectra of strong-coupling large polarons and its display in cuprates," Physical Review B, vol. 77, no. 16, Article ID 165136, 11 pages, 2008.

[251] A. S. Mishchenko, unpublished.

[252] E. N. Myasnikov, A. E. Myasnikova, and Z. P. Mastropas, "Multiphonon generation during photodissociation of slow Landau-Pekar polarons," Zhurnal Éksperimental'nŏ i Teoreticheskoŭ Fiziki, vol. 129, no. 3, pp. 548-565, 2006.

[253] E. N. Myasnikov, A. E. Myasnikova, and Z. P. Mastropas, "Multiphonon generation during photodissociation of slow Landau-Pekar polarons," Journal of Experimental and Theoretical Physics, vol. 102, no. 3, pp. 480-496, 2006.

[254] L. Vidmar, J. Bonča, and S. Maekawa, "Optical conductivity in the $t$-J Holstein model," Physical Review B, vol. 79, no. 12, Article ID 125120, 6 pages, 2009.

[255] A. Macridin, B. Moritz, M. Jarrell, and T. Maier, "Synergistic polaron formation in the Hubbard-Holstein model at small doping," Physical Review Letters, vol. 97, no. 5, Article ID 056402, 4 pages, 2006.

[256] A. Ramšak and P. Horsch, "Spin polarons in the $t-J$ model: shape and backflow," Physical Review B, vol. 48, no. 14, pp. 10559-10562, 1993.

[257] W. F. Brinkman and T. M. Rice, "Single-particle excitations in magnetic insulators," Physical Review B, vol. 2, no. 5, pp. 1324-1338, 1970.

[258] V. Cataudella, G. De Filippis, F. Martone, and C. A. Perroni, "Variational approach to the optimized phonon technique for electron-phonon problems," Physical Review B, vol. 70, no. 19, Article ID 193105, 4 pages, 2004. 
[259] G. De Filippis, V. Cataudella, V. M. Ramaglia, and C. A. Perroni, "Static and dynamic polaron features in a coherentstate basis," Physical Review B, vol. 72, no. 1, Article ID 014307, 7 pages, 2005.

[260] A. Fujimori, A. E. Bocquet, K. Morikawa, et al., "Electronic structure and electron-phonon interaction in transition metal oxides with d0 configuration and lightly doped compounds," Journal of Physics and Chemistry of Solids, vol. 57, no. 10, pp. 1379-1384, 1996.

[261] C. A. Perroni, V. Cataudella, and G. De Filippis, "Polaron features for long-range electron-phonon interaction,” Journal of Physics: Condensed Matter, vol. 16, no. 9, pp. 1593-1601, 2004.

[262] M. Berciu and G. A. Sawatzky, "Light polarons and bipolarons for a highly inhomogeneous electron-boson coupling," Europhysics Letters, vol. 81, no. 5, Article ID 57008, 6 pages, 2008.

[263] J. Bonča and S. A. Trugman, "Bipolarons in the extended Holstein Hubbard model," Physical Review B, vol. 64, no. 9, Article ID 094507, 4 pages, 2001.

[264] A. S. Alexandrov and B. Ya. Yavidov, "Small adiabatic polaron with a long-range electron-phonon interaction," Physical Review B, vol. 69, no. 7, Article ID 073101, 4 pages, 2004.

[265] J. T. Devreese and A. S. Alexandrov, "Fröhlich polaron and bipolaron: recent developments," Reports on Progress in Physics, vol. 72, no. 6, Article ID 066501, 52 pages, 2009.

[266] M. Zoli, "Two- and three-dimensional polaronic motion: beyond the Holstein model," Physical Review B, vol. 57, no. 17, pp. 10555-10559, 1998.

[267] M. Zoli, "Lattice-dynamics effects on small-polaron properties,” Physical Review B, vol. 61, no. 21, pp. 14523-14530, 2000.

[268] M. Zoli and A. N. Das, "Polaron crossover in molecular solids," Journal of Physics: Condensed Matter, vol. 16, no. 21, pp. 3597-3607, 2004.

[269] A. S. Alexandrov, Theory of Superconductivity: From Weak to Strong Coupling, Institute of Physics Publishing, Bristol, UK.

[270] A. S. Alexandrov, "Bipolaren anisotropic flat bands, Hall mobility edge, and metal-semiconductor duality of overdoped high- $T_{c}$ oxides," Physical Review B, vol. 53, no. 5, pp. 2863-2869, 1996.

[271] A. S. Alexandrov and P. E. Kornilovitch, "The FröhlichCoulomb model of high-temperature superconductivity and charge segregation in the cuprates," Journal of Physics: Condensed Matter, vol. 14, no. 21, pp. 5337-5348, 2002.

[272] J. P. Hague, P. E. Kornilovitch, J. H. Samson, and A. S. Alexandrov, "Superlight small bipolarons in the presence of a strong coulomb repulsion," Physical Review Letters, vol. 98, no. 3, Article ID 037002, 4 pages, 2007.

[273] H. Fehske, J. Loos, and G. Wellein, "Lattice polaron formation: effects of nonscreened electron-phonon interaction," Physical Review B, vol. 61, no. 12, pp. 8016-8025, 2000.

[274] T. Sakai, D. Poilblanc, and D. J. Scalapino, "Hole pairing and phonon dynamics in generalized two-dimensional $t-J$ Holstein models," Physical Review B, vol. 55, no. 13, pp. 8445-8451, 1997.

[275] C. Slezak, A. Macridin, G. A. Sawatzky, M. Jarrell, and T. A. Maier, "Spectral properties of Holstein and breathing polarons," Physical Review B, vol. 73, no. 20, Article ID 205122, 10 pages, 2006.

[276] O. Rösch and O. Gunnarsson, "Apparent electron-phonon interaction in strongly correlated systems," Physical Review Letters, vol. 93, no. 23, Article ID 237001, 4 pages, 2004.
[277] O. Rösch, G. Sangiovanni, and O. Gunnarsson, "Sum rules and vertex corrections for electron-phonon interactions," Physical Review B, vol. 75, no. 3, Article ID 035119, 9 pages, 2007.

[278] M. Capone, G. Sangiovanni, C. Castellani, C. Di Castro, and M. Grilli, "Phase separation close to the density-driven mott transition in the Hubbard-Holstein model," Physical Review Letters, vol. 92, no. 10, Article ID 106401, 4 pages, 2004.

[279] G. Sangiovanni, M. Capone, C. Castellani, and M. Grilli, "Electron-phonon interaction close to a mott transition," Physical Review Letters, vol. 94, no. 2, Article ID 026401, 4 pages, 2005.

[280] G. Sangiovanni, M. Capone, and C. Castellani, "Relevance of phonon dynamics in strongly correlated systems coupled to phonons: dynamical mean-field theory analysis," Physical Review B, vol. 73, no. 16, Article ID 165123, 9 pages, 2006.

[281] G. Sangiovanni, O. Gunnarsson, E. Koch, C. Castellani, and M. Capone, "Electron-phonon interaction and antiferromagnetic correlations," Physical Review Letters, vol. 97, no. 4, Article ID 046404, 4 pages, 2006.

[282] J. Zhong and H.-B. Schüttler, "Polaronic anharmonicity in the Holstein-Hubbard model," Physical Review Letters, vol. 69, no. 10, pp. 1600-1603, 1992.

[283] H. Fehske, H. Röder, G. Wellein, and A. Mistriotis, "Holepolaron formation in the two-dimensional Holstein $t-J$ model: a variational Lanczos study," Physical Review B, vol. 51, no. 23, pp. 16582-16593, 1995.

[284] G. Wellein, H. Röder, and H. Fehske, "Polarons and bipolarons in strongly interacting electron-phonon systems," Physical Review B, vol. 53, no. 15, pp. 9666-9675, 1996.

[285] H. Matsueda, T. Tohyama, and S. Maekawa, "Electronphonon coupling and spin-charge separation in onedimensional Mott insulators," Physical Review B, vol. 74, no. 24, Article ID 241103, 4 pages, 2006.

[286] H. Matsueda, A. Ando, T. Tohyama, and S. Maekawa, "Enhancement of phonon effects in photoexcited states of one-dimensional Mott insulators," Physical Review B, vol. 77, no. 19, Article ID 193112, 4 pages, 2008.

[287] T. Hanaguri, C. Lupien, Y. Kohsaka, et al., "A 'checkerboard' electronic crystal state in lightly holedoped $\mathrm{Ca}_{2-x} \mathrm{Na}_{x} \mathrm{CuO}_{2} \mathrm{Cl}_{2}$," Nature, vol. 430, no. 7003, pp. 1001-1005, 2004.

[288] Y. Kohsaka, C. Taylor, K. Fujita, et al., "An intrinsic bondcentered electronic glass with unidirectional domains in underdoped cuprates," Science, vol. 315, no. 5817, pp. 13801385, 2007.

[289] S. H. Pan, J. P. O’Neal, R. L. Badzey, et al., "Microscopic electronic inhomogeneity in the high- $T_{c}$ superconductor $\mathrm{Bi}_{2} \mathrm{Sr}_{2} \mathrm{CaCu}_{2} \mathrm{O}_{8+x}$," Nature, vol. 413, no. 6853, pp. 282-285, 2001.

[290] P. M. Singer, A. W. Hunt, and T. Imai, " ${ }^{63} \mathrm{Cu}$ NQR evidence for spatial variation of hole concentration in $\mathrm{La}_{2-x} \mathrm{Sr}_{x} \mathrm{CuO}_{4}$," Physical Review Letters, vol. 88, no. 4, Article ID 047602, 4 pages, 2002.

[291] N. Pavlenko and T. Kopp, "Electron-phonon coupling in a two-dimensional inhomogeneous electron gas: consequences for surface spectral properties," Journal of Physics: Condensed Matter, vol. 20, no. 39, Article ID 395203, 7 pages, 2008.

[292] N. Pavlenko and T. Kopp, "Interface controlled electronic charge inhomogeneities in correlated heterostructures," Physical Review Letters, vol. 97, no. 18, Article ID 187001, 4 pages, 2006. 
[293] J. P. Hague, P. E. Kornilovitch, and A. S. Alexandrov, "Trapping of lattice polarons by impurities," Physical Review B, vol. 78, no. 9, Article ID 092302, 4 pages, 2008.

[294] S. M. Girvin and M. Jonson, "Dynamical electron-phonon interaction and conductivity in strongly disordered metal alloys," Physical Review B, vol. 22, no. 8, pp. 3583-3597, 1980.

[295] F. X. Bronold and H. Fehske, "Anderson localization of polaron states," Physical Review B, vol. 66, no. 7, Article ID 073102, 4 pages, 2002.

[296] F. X. Bronold, A. Alvermann, and H. Fehske, "Anderson localization in strongly coupled disordered electron-phonon systems," Philosophical Magazine, vol. 84, no. 7, pp. 673-704, 2004.

[297] A. Alvermann and H. Fehske, "Chebyshev approach to quantum systems coupled to a bath," Physical Review B, vol. 77, no. 4, Article ID 045125, 17 pages, 2008.

[298] M. Berciu, A. S. Mishchenko, and N. Nagaosa, "Holstein polaron in the presence of disorder," http://arxiv.org/ abs/0906.1233.

[299] I. I. Mazin, E. G. Maksimov, S. N. Rashkeev, S. Y. Savrasov, and Y. A. Uspenskii, "Optical properties of metal oxides of the $\mathrm{La}_{2} \mathrm{CuO}_{4}$ type," Pisma Zh. Ehksp. Teor. Fiz, vol. 47, p. 94, 1987, English translation in Soviet Physics-JETP Letters, vol. 47, p. 113, 1988.

[300] E. G. Maksimov, S. N. Rashkeev, S. Yu. Savrasov, and Yu. A. Uspenskii, "Microscopic studies of the optical spectra of $\mathrm{YBa}_{2} \mathrm{Cu}_{3} \mathrm{O}_{7}$," Physical Review Letters, vol. 63, no. 17, pp. 1880-1883, 1989.

[301] S. N. Rashkeev, et al., "Electronic structure and optical properties of $\mathrm{YBa}_{2} \mathrm{Cu}_{3} \mathrm{O}_{7}$," Zhurnal Éksperimental'noй $i$ Teoreticheskoŭ Fiziki, vol. 97, p. 1688, 1990, translated in Soviet Physics. JETP, vol. 70, p. 952, 1990.

[302] S. V. Shulga, O. V. Dolgov, and E. G. Maksimov, "Electronic states and optical spectra of HTSC with electron-phonon coupling," Physica C, vol. 178, no. 4-6, pp. 266-274, 1991.

[303] E. G. Maksimov, H. J. Kaufmann, E. K. H. Salje, Y. De Wilde, N. Bontemps, and J. P. Contour, "Comparative analysis of the optical spectra of $\mathrm{YBa}_{2} \mathrm{Cu}_{3} \mathrm{O}_{7}$," Solid State Communications, vol. 112, no. 8, pp. 449-454, 1999.

[304] T. Jarlborg, "Weak screening of high frequency phonons and superconductivity in $\mathrm{YBa}_{2} \mathrm{Cu}_{3} \mathrm{O}_{7}$," Solid State Communications, vol. 71, no. 8, pp. 669-671, 1989.

[305] R. E. Cohen, W. E. Pickett, and H. Krakauer, "Theoretical determination of strong electron-phonon coupling in $\mathrm{YBa}_{2} \mathrm{Cu}_{3} \mathrm{O}_{7}, "$ Physical Review Letters, vol. 64, no. 21, pp. 2575-2578, 1990.

[306] R. Zeyher, "Improtance of long-range electron-phonon coupling in high- $T_{c}$ superconductors," Zeitschrift für Physik $B$, vol. 80, no. 2, pp. 187-192, 1990.

[307] H. Krakauer, W. E. Pickett, and R. E. Cohen, "Large calculated electron-phonon interactions in $\mathrm{La}_{2-x} \mathrm{M}_{x} \mathrm{CuO}_{4}$," Physical Review B, vol. 47, no. 2, pp. 1002-1015, 1993.

[308] E. G. Maksimov, "High-temperature superconductivity: the current state," Physics-Uspekhi, vol. 43, no. 10, pp. 965-990, 2000, Russian translation in Uspekhi Fizicheskikh Nauk, vol. 170, p. 1033, 2000.

[309] O. V. Dolgov and S. V. Shulga, "Analysis of intermediate boson spectra from FIR data for HTSC and heavy fermion systems," Journal of Superconductivity, vol. 8, no. 5, pp. 611612, 1995.

[310] J. P. Carbotte, E. Schachinger, and D. N. Basov, "Coupling strength of charge carriers to spin fluctuations in hightemperature superconductors," Nature, vol. 401, no. 6751, pp. 354-356, 1999. 

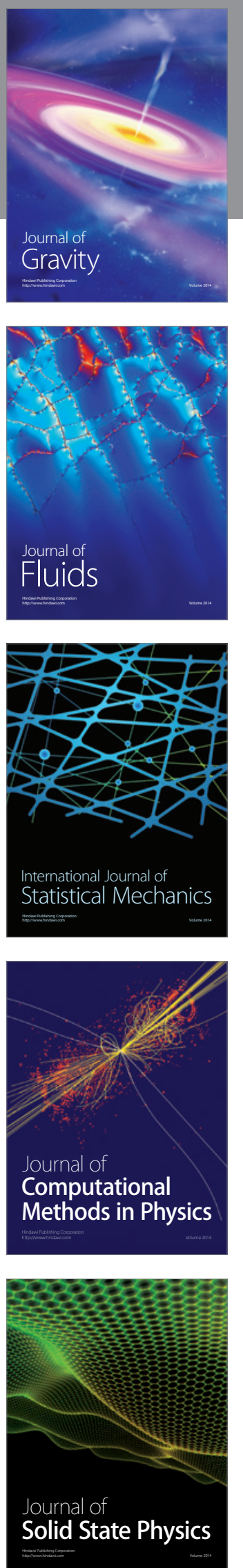

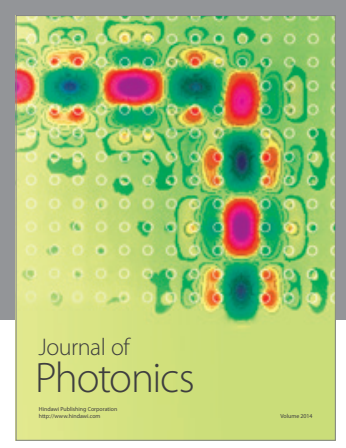

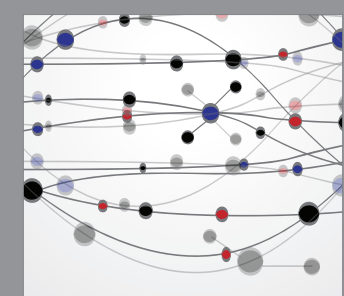

The Scientific World Journal
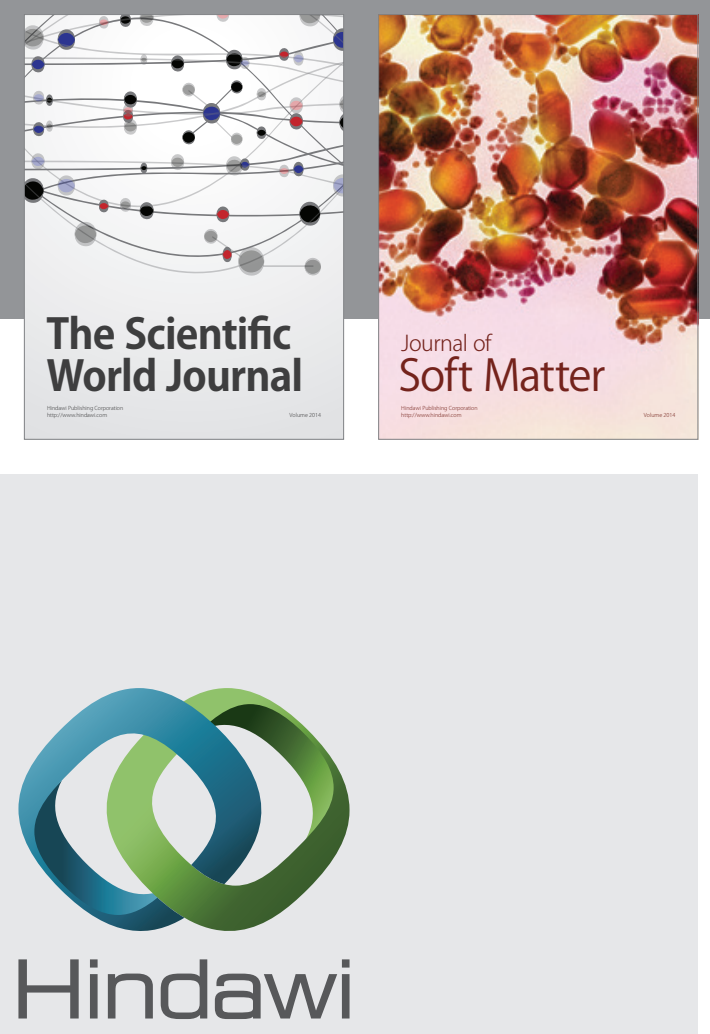

Submit your manuscripts at

http://www.hindawi.com
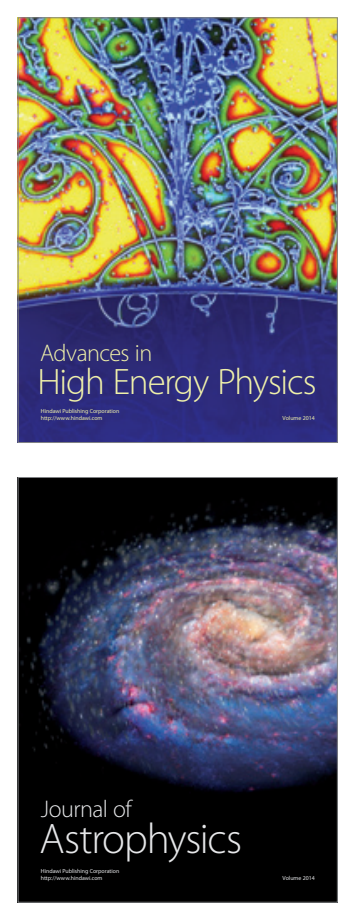
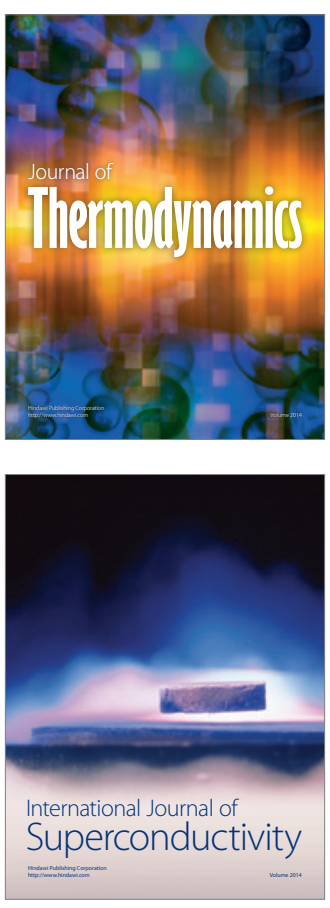
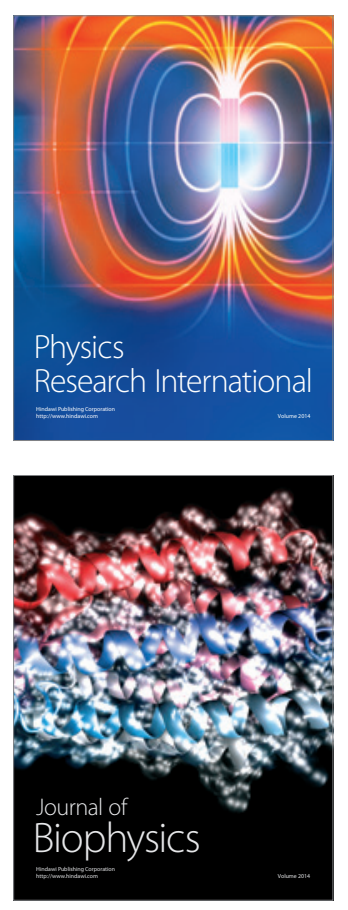
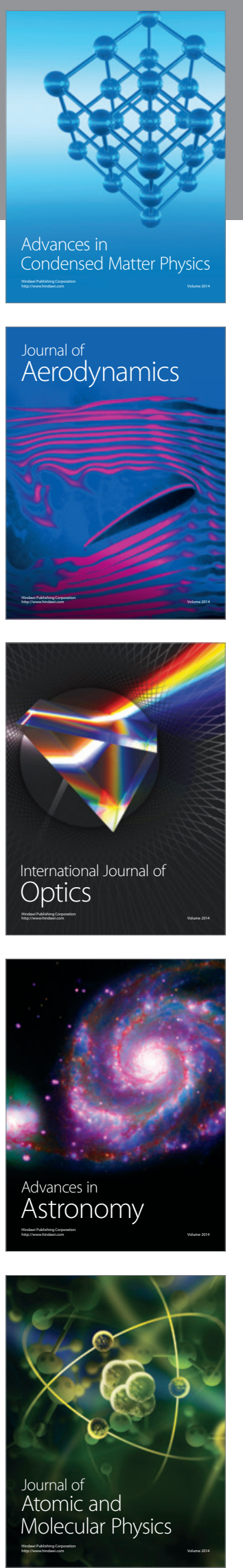